\author{
Universidade de São Paulo \\ Instituto de Física
}

\title{
Laços de Wilson Supersimétricos na correspondência AdS/CFT
}

\author{
DhyanVictor Hiromitsu Kuraoka \\ Orientador: Prof. Dr. Diego Trancanelli
}

Banca Examinadora:

Prof. Dr. Diego Trancanelli (IFUSP)

Prof. Dr. Victor de Oliveira Rivelles (IFUSP)

Prof. Dr. Horatiu Stefan Nastase (IFT-UNESP)

Dissertação de mestrado apresentada ao Instituto de Física para a obtenção do título de Mestre em Ciências 
FICHA CATALOGRÁFICA

Preparada pelo Serviço de Biblioteca e Informação do Instituto de Física da Universidade de São Paulo

Kuraoka, Dhyan Victor Hiromitsu

Laços de Wilson supersimétricos na correspondência AdS/CFT - São Paulo, 2013.

Dissertação (Mestrado) - Universidade de São Paulo. Instituto de Física - Depto. de Física Matemática

Orientador: Prof Dr Diego Trancanelli

Área de Concentração: Física teórica

Unitermos: 1. Teoria de cordas; 2. Física teórica;

3. Física matemática.

USP/IF/SBI-061/2013 


\section{Agradecimentos}

Agradeço a todos os meus colegas que me acompanharam durante a minha vida acadêmica, me apoiando e incentivando. Durante este período tive a grande oportunidade de dividir a maior parte do meu tempo na companhia deles, tão especiais, o que certamente foi crucial em diversos momentos bons e ruins que vivi.

Um agradecimento ao prof. Diego Trancanelli, por sua orientação. Este que teve paciência e dedicação em seu trabalho de me orientar.

Agradecimento aos prof.'s Manoel Roberto Robilotta e Gustavo Burdman, que me inspiraram em como olhar para a física e me lembraram de como desfrutar desta bela construção humana.

A minha família.

Agradecimento especial a Mariana Franco.

A CAPES e FAPESP pelo apoio financeiro. 


\section{Resumo}

O objetivo desta dissertação é revisar os operadores laços de Wilson no contexto da correspondência AdS/CFT. Estes operadores, presentes em qualquer teoria de calibre, são importantes por nos fornecer um parâmetro de ordem para a transição de fase confinante/desconfinante. Além disso, eles são particularmente importantes no estudo da correspondência AdS/CFT pois: i) Eles nos dão, em alguns casos, resultados exatos graças ao fato de poderem ser localizados em um modelo de matrizes, desta forma nos permitindo fazer testes altamente não triviais da correspondência; $i i)$ Eles são os objetos da teoria de calibre que são duais a cordas propagando no interior do espaço, nos dando um rico dicionário entre quantidades no interior ( $\mathrm{AdS}$ ) e na borda do espaço (CFT).

Depois de revisarmos os laços de Wilson em teorias de calibre e a correspondência AdS/CFT, introduziremos a definição dos laços de Wilson supersimétricos 1/2 BPS. Calcularemos eles para o caso de um acoplamento fraco e para qualquer outro valor da constante de acoplamento usando técnicas de modelos de matrizes. Finalmente, compararemos nossos resultados com computações de superfícies minimais no interior do espaço, encontrando uma concordância perfeita.

Palavras-chave Laços de Wilson, teoria de cordas, correspondência AdS/CFT, teorias de calibre supersimétricas, modelos de matrizes, cordas em AdS. 


\section{Abstract}

The aim of this thesis is to review Wilson loop operators in the context of the AdS/CFT correspondence. These operators, which are present in any gauge theory, are important because they furnish an order parameter for confinement/deconfinement phase transitions. Besides this, they are particularly relevant in the study of the AdS/CFT correspondence

because: i) they allow, in some cases, for exact results thanks to localization to matrix models and make it possible to perform highly non-trivial tests of the correspondence; ii) they are the gauge theory objects dual to strings propagating in the bulk of the space and give a rich dictionary between bulk (AdS) and boundary (CFT) quantities.

After reviews of Wilson loops in gauge theories and of the AdS/CFT correspondence, we will introduce the definition of $1 / 2$ BPS supersymmetric Wilson loops, we will compute them at weak coupling and then at any order in the coupling constant via matrix model techniques, and finally we will compare our results with minimal surface computations in the bulk, finding perfect agreement.

Keywords Wilson loops, string theory, AdS/CFT correspondence, supersymmetric gauge theories, matrix models, strings in AdS space. 


\section{Sumário}

$\begin{array}{lll}\text { Agradecimentos } & \text { i }\end{array}$

Resumo $\quad$ ii

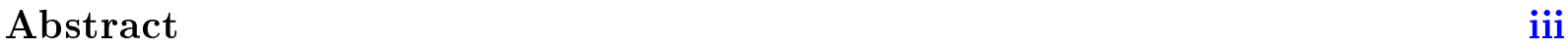

Índice $\quad$ iv

Lista de Figuras $\quad$ vii

1 Introdução 1

1.1 Laços de Wilson holográficos . . . . . . . . . . . . . . . 3

2 Laços de Wilson em teorias de calibre $\quad 8$

2.1 Links de Wilson . . . . . . . . . . . . . . . . 8

2.2 Invariância de calibre . . . . . . . . . . . . . . . 10

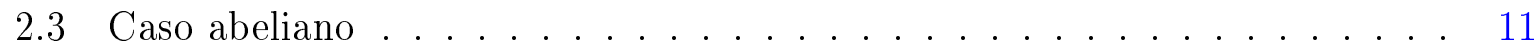

2.4 Caso não abeliano . . . . . . . . . . . . . . . . 12

3 Revisão da correspondência AdS/CFT $\quad 17$

3.1 Considerações gerais . . . . . . . . . . . . . . . . 17

3.1.1 Exemplo da correspondência AdS/CFT . . . . . . . . . 20

3.1.2 Contando os graus de liberdade . . . . . . . . . . . 21

3.1.3 O limite de ${ }^{\prime} t$ Hooft . . . . . . . . . . . . . . 23

3.2 A conjectura de Maldacena . . . . . . . . . . . . . . 28

3.2.1 D-branas e a conjectura . . . . . . . . . . . . 28 
$3.3 \mathcal{N}=4$ super Yang-Mills . . . . . . . . . . . . . . . 34

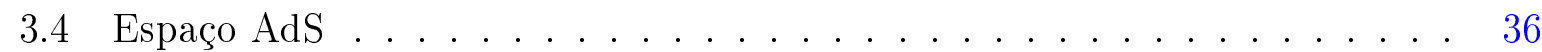

3.5 Verificações das simetrias globais ................ 40

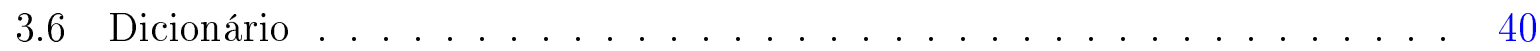

4 Laços de Wilson supersimétricos $\quad 44$

4.1 Definindo o laço de Wilson supersimétrico . . . . . . . . . . . . 44

4.2 Condições de supersimetria . . . . . . . . . . . . . . . 45

4.3 Laços de Wilson em teoria perturbativa . . . . . . . . . . . . 47

4.3.1 Divergência do propagador . . . . . . . . . . . . 49

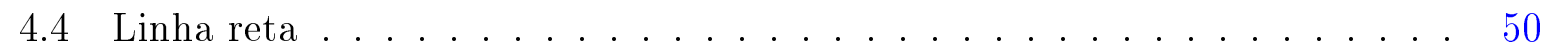

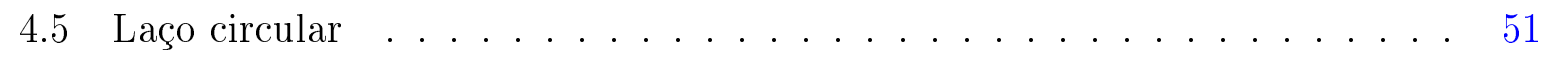

4.5.1 Soma dos gráficos planares ................. 51

5 Resultados exatos via modelos de matrizes $\quad 55$

5.1 Prova de Pestun via localização . . . . . . . . . . . . . 56

5.2 Modelo de matrizes . . . . . . . . . . . . . . . 58

5.3 Aproximação por ponto de sela . . . . . . . . . . . . . 63

5.4 Polinômios ortogonais . . . . . . . . . . . . . 67

6 Laços de Wilson holográficos $\quad 71$

6.1 Superfícies minimais ............................ 71

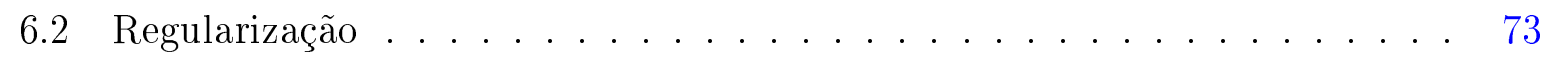

6.3 Cálculos para linha e círculo e comparação com o modelo de matrizes . . 74

6.3.1 Cálculo da linha ...................... 74

6.3.2 Cálculo do laço circular . . . . . . . . . . . . 75

6.4 Linhas antiparalelas . . . . . . . . . . . . . 77

$\begin{array}{ll}\text { Conclusões } & 82\end{array}$

$\begin{array}{lr}\text { Apêndice I } & 84\end{array}$

$\begin{array}{ll}\text { Apêndice II } & 86\end{array}$ 
$\begin{array}{ll}\text { Apêndice III } & 87\end{array}$

Apêndice IV $\quad 89$

$\begin{array}{ll}\text { Bibliografia } & 92\end{array}$ 


\section{Lista de Figuras}

1.1 Um laço de Wilson supersimétrico na teoria de calibre (o contorno pontilhado na figura) corresponde a uma superfície embutida no interior do espaço e com a borda ao longo do contorno do operador da teoria de calibre. 6

2.1 Uma plaqueta centrada em $x$ e de lado $a$, com as direções 1 e 2 explicitadas. 13

2.2 Ilustração da terceira propriedade em $(2.29) \ldots \ldots \ldots \ldots \ldots \ldots$

3.1 Rede de tamanho $R$, onde introduzimos um corte $\mathrm{UV}, \delta$, para regularizar o número de graus de liberdade na TQC. . . . . . . . . . . . . . . . 22

3.2 Regularização do número de graus de liberdade no interior da AdS. . . . 23

3.3 Propagador da teoria em notação de linha dupla. . . . . . . . . . . . . . 24

3.4 Vértice triplo (a) e quadruplo (b) em notação de linhas duplas, proporcio-

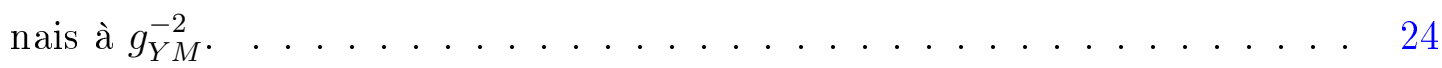

3.5 Exemplos de diagramas em notação de linhas duplas com suas respectivas contribuições. . . . . . . . . . . . . . . . . . . . 25

3.6 Diagramas em notação de linha dupla com suas respectivas superfícies de Riemann. . . . . . . . . . . . . . . . 26

3.7 Diagramas em notação de linha dupla para férmions com sua respectivas superfícies de Riemann. . . . . . . . . . . . . . . . . 27

3.8 Expansão em genus na teoria de cordas. . . . . . . . . . . . . . 27

3.9 Construção de teorias não abelianas através de um conjunto de branas. . 30

3.10 Espectro das cordas separadas pela 'garganta' de AdS. . . . . . . . . . . . 33

3.11 Espaço AdS como um hiperboloide nas coordenadas globais $\rho, t$ e $\Omega_{k}$. . . 38 
3.12 Espaço AdS como um cilindro em coordenadas $\theta, t$ e $\Omega_{k}$, e uma representação de uma trajetória do tipo luz atingindo a borda de AdS. . . . . . . . . 39

4.1 Representação diagramática de $C_{1}$, onde o propagador representa ambos os campos, escalar e de calibre, enquanto o círculo representa o contorno do

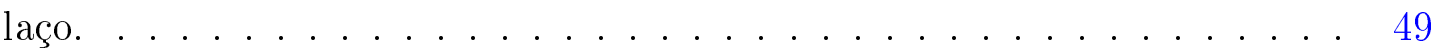

4.2 Cancelamento dos termos de vértices e correções a um loop do propagador. 52

4.3 Diagramas 'rainbow' ou 'ladders', cada diagrama com $n$ propagadores será porporcional à $\left(g_{Y M}^{2}\right)^{n} \ldots \ldots \ldots \ldots . \ldots \ldots$

5.1 Gráfico da distribuição de autovalores do modelo de matrizes gaussiano, a lei do semicírculo de Wigner-Dyson. . . . . . . . . . . . . . . 66

6.1 Podemos construir objetos na representação fundamental distanciando uma D3-brana de um conjunto de $N$ D3-branas, como ilustrado acima. . . . . 72

6.2 Superfície minimal correspondente ao contorno circular. . . . . . . . . . 77

6.3 Superfície minimal correspondente ao contorno retangular. . . . . . . . . 79 


\section{Capítulo 1}

\section{Introdução}

Um dos aspectos mais fascinantes da teoria das cordas é a ideia de holografia, ou seja, a ideia de que a teoria das cordas, que é uma teoria de gravitação quântica, tenha uma descrição equivalente em termos de uma teoria quântica de campos (sem gravidade) definida na fronteira do espaço onde vivem as cordas. A realização mais notável e explícita desta concepção é a correspondência "Anti de Sitter/Conformal Field Theory" (AdS/CFT), proposta por Maldacena em 1997 [1]. ${ }^{1}$ Esta tem sido realmente um dos desenvolvimentos mais inovadores em teoria das cordas das últimas duas décadas. Revolucionou nosso campo de pesquisa, abrindo diversas novas vias de investigação e oferecendo-nos uma nova e poderosa ferramenta para a resolução de problemas tanto em teoria de campos quanto na gravidade quântica.

No exemplo mais estudado da correspondência, temos um espaço-tempo de dez dimensões que é o produto de uma esfera e de uma componente não compacta chamada espaço de Anti de Sitter (daí deriva o AdS no nome da correspondência). A teoria, quando definida no interior (ou "bulk") deste espaço, contém cordas fechadas que descrevem a gravidade; enquanto que definida na fronteira quadri-dimensional do espaço (ou "boundary") torna-se uma teoria de calibre altamente simétrica e conforme (o CFT no nome) sem gravidade. Em termos mais técnicos, segundo a correspondência AdS/CFT, a teoria de calibre maximamente supersimétrica, chamada de $\mathcal{N}=4$ super Yang-Mills em quatro

\footnotetext{
${ }^{1}$ Outros trabalhos iniciais muito importantes são [2,3]. Resumos da correspondência AdS/CFT incluem, por exemplo, [4-8].
} 
dimensões com grupo de calibre $S U(N)$ é exatamente equivalente às supercordas de tipo IIB no espaço-tempo de dez dimensões $A d S_{5} \times S^{5}$, ou seja, produto direto de um espaço Anti-de Sitter em cinco dimensões com uma esfera cinco dimensional, com $N$ unidades de fluxo atravessando a $S^{5}$.

Essa dualidade mostra-se bastante útil por tornar possível tanto a utilização de uma descrição ao invés de outra para computação de quantidades físicas quanto a reformulação de perguntas em uma ou outra linguagem. De fato, ambas as descrições, do bulk e do boundary, são complementares, de modo que problemas de difícil análise em uma delas são frequentemente simples na outra e vice-versa.

Utilizando-se a descrição do bulk para aprender sobre a teoria do boundary, pode-se demonstrar que um limite não trivial e fortemente acoplado da teoria de calibre é mapeado para um limite da teoria das cordas fracamente acoplado e solúvel. De fato, quando toma-se o limite de um grande número de cores $N$ e grande constante de acoplamento $\lambda=g_{Y M}^{2} N$ na teoria de calibre, a correspondente teoria das cordas é bem aproximada pela supergravidade clássica. Esta mostra-se, portanto, uma poderosa ferramenta para o estudo de situações fortemente acopladas, nas quais métodos perturbativos não são diretamente aplicáveis.

É claro que $\mathcal{N}=4$ super Yang-Mills é uma teoria altamente supersimétrica e conforme, que difere em muitos aspectos das teorias de calibre do mundo real, tais como a Cromodinâmica Quântica (QCD), que descreve a interação forte entre as partículas nos núcleos atômicos. Apesar disso, a correspondência AdS/CFT nos fornece diversas novas ferramentas e conhecimentos necessários para compreender sistemas fortemente acoplados, permitindo-nos obter alguns resultados universais que não dependem muito dos detalhes da teoria, especialmente quando esta encontra-se a temperatura finita. O objetivo final dessa direção investigativa é de resolver problemas complexos e não perturbativos de teorias quânticas de campo, como o confinamento na QCD, usando a teoria das cordas. Na verdade, encontrar o equivalente à QCD em teoria das cordas nos permitirá provavelmente entender o confinamento e o regime fortemente acoplado da teoria

Podemos também fazer o caminho oposto, do bulk ao boundary, e usar o que sabemos na teoria de calibre para melhor entender as características da gravidade. A correspon- 
dência AdS/CFT é, de fato, o instrumento mais eficaz e promissor do qual hoje dispomos para a investigação de problemas profundos e importantes de gravidade quântica. Num exemplo concreto, a correspondência AdS/CFT deu-nos um novo ponto de vista sobre o paradoxo da informação na física dos buracos negros quânticos. Este paradoxo, formulado por S. Hawking há mais de 30 anos [9], baseia-se no desaparecimento aparente de informações que caem em um buraco negro, com estados puros evoluindo para estados mistos, em contraste com as leis da mecânica quântica. Usando a correspondência AdS/CFT podemos, em princípio, entender este paradoxo reformulando-o na linguagem equivalente da teoria quântica de campo, onde a evolução dos estados é unitária, não ocorrendo perda alguma de informação.

\subsection{Laços de Wilson holográficos}

Uns dos observáveis mais importantes em qualquer teoria de campos são as funções de correlação de operadores conhecidos como laços de Wilson. Estes são operadores que estão presentes em qualquer teoria de calibre e são definidos como a holonomia do campo de calibre da teoria, $A_{\mu}$, ao redor de algum contorno $C$

$$
W_{R}(C)=\operatorname{Tr}_{R} \mathcal{P} \exp \oint_{C} d \tau i \dot{x}^{\mu} A_{\mu}
$$

Aqui, $R$ é uma representação do grupo de calibre sobre o qual o laço de Wilson se transforma, e $\operatorname{Tr}_{R}$ é o traço sobre tal representação. O símbolo $\mathcal{P}$ significa que a integral precisa ser ordenado, $x^{\mu}(\tau)$ parametriza o laço $C$. Fisicamente, o operador na eq. (1.1) representa o fator de fase de uma partícula de 'carga' $R$ movimentando-se ao longo do contorno $C$. Da definição acima, fica claro que um laço de Wilson não é definido em um único ponto do espaço, mas sim sobre uma curva uni-dimensional. Por esta razão, é dito que este é um operador não local.

Laços de Wilson são quantidades extremamente interessantes de se calcular porque eles podem ser interpretados como parâmetros de ordem para a transição de fase de confinamento/desconfinamento, ou seja, eles nos oferecem um critério para entender se uma partícula de uma teoria pode existir separadamente de outras ou precisa estar fortemente ligada (ou confinada) em uma composição de estados, como é o caso de quarks e glúons 
da QCD, os quais são confinados em bárions e mesons. Mais especificamente, o potencial $V(D)$ entre duas partículas pesadas carregadas a uma distância $D$ uma da outra é dado pelo valor esperado do laço de Wilson retangular, ${ }^{2}$ definido por duas linhas paralelas de comprimento $T$ e separação $D$

$$
\lim _{T \rightarrow \infty}\langle W\rangle=e^{-T V(D)} .
$$

Uma teoria na fase de confinamento exibe um potencial linear entre as duas cargas, $V(D) \propto D$. O expoente na eq. (1.2) assim escala como a área do laço retangular, um comportamento referido como lei da área.

Além de serem importantes por essa razão, laços de Wilson também atuam em um papel central na correspondência AdS $/ \mathrm{CFT}^{3}{ }^{3}$ No contexto desta correspondência, nós somos mais interessados em teorias de calibre supersimétricas, de modo que somos levados a considerar generalizações do operador (1.1), os quais também preservam alguma supersimetria. Estas generalizações são chamados de laços de Wilson supersimétricos. Os laços de Wilson supersimétricos mais estudados são os laços chamados de 1/2 BPS na teoria de $\mathcal{N}=4$ super Yang-Mills em quatro dimensões com grupo de calibre $S U(N)$. Esses operadores são definidos ao longo de uma linha reta infinita ou em um círculo, ambos preservando metade das supercargas da teoria. Eles incluem em suas definições, além do campo de calibre como na eq. (1.1), também os campos escalar adjuntos $\Phi^{i}(i=1, \ldots, 6)$ da teoria

$$
W_{R}(C)=\operatorname{Tr}_{R} \mathcal{P} \exp \oint_{C} d \tau\left(i \dot{x}^{\mu} A_{\mu}(\tau)+|\dot{x}| \theta^{i} \Phi^{i}(\tau)\right)
$$

Aqui $C$, como foi dito, é uma linha reta infinita ou um círculo e $\theta^{i}$ é um vetor unitário constante.

O estudo de laços de Wilson supersimétricos na correspondência AdS/CFT é muito importante, principalmente por duas razões: Eles oferecem verificações não triviais da correspondência e eles também nos dão um rico dicionário entre estados na teoria de calibre e estados na teoria de cordas, como explicaremos agora.

Primeiramente, a computação de valores esperados e funções de correlação dos laços de Wilson nos dá um teste muito poderoso da correspondência AdS/CFT. Como men-

\footnotetext{
${ }^{2}$ Para mais detalhes deste fato [10].

${ }^{3}$ Para um resumo, ver [11].
} 
cionamos acima, esta correspondência é uma dualidade de "acoplamento forte/fraco", ou seja, enquanto um lado da dualidade é fracamente acoplado (a assim fácil de se calcular) o outro lado é fortemente acoplado, e vice-versa. Isto faz da correspondência algo interessante, porque ela nos permite mapear problemas difíceis de um lado em tratáveis no outro, mas, ao mesmo tempo, isso também faz com que seja difícil de prová-la (ou negá-la), pois técnicas de computação realizáveis nos dois lados da dualidade não possuem uma intersecção de seus domínios de validade. Entretanto é importante testar esta correspondência sempre que possível. Para fazer estes testes, nós precisamos, por exemplo, sermos capazes de calcular algumas quantidade na teoria de calibre no regime de acoplamento forte (que é o regime onde técnicas comuns da teoria de perturbação não são válidas).

Acontece que é possível calcular exatamente as funções de correlação de certos laços de Wilson supersimétricos, o que significa que é possível obter resultados que são válidos para qualquer valor da costante de acoplamento da teoria de calibre e qualquer $N$ do grupo $S U(N)$. Isto graças à poderosa técnica computacional conhecida como localização [12], que permite em alguns casos expressar a integral de caminho quadri-dimensional da teoria em termos de um modelo de matriz zero-dimensional muito mais simples, que é completamente solúvel. De fato é possível mostrar, por exemplo, que o valor esperado do operador (circular) 1/2 BPS na eq. (1.3) é dado pela seguinte integral de matrizes

$$
\left\langle W_{R}\right\rangle=\int d M \exp \left(-\frac{2}{g_{Y M}^{2}} \operatorname{Tr} M^{2}\right) \operatorname{Tr}_{R} e^{M},
$$

onde $M$ é uma matriz hermitiana $N \times N$ e $g_{Y M}$ é a constante de acoplamento da teoria. Usando técnicas bem conhecidas de modelos de matrizes, esta integral pode ser resolvida exatamente para qualquer valor de $N$ e $g_{Y M}$, chegando no resultado (5.79) do Capítulo 5 . Interpolando o resultado exato que se obtém desta maneira para grande $N$ e grande $\lambda$, pode-se comparar este cálculo da teoria de calibre com um cálculo correspondente da teoria de cordas em acoplamento fraco no interior do espaço. Esta comparação nos permite fazer uma checagem altamente não trivial da correspondência AdS/CFT.

Uma segunda razão do porquê os laços de Wilson estão no centro dos estudos da correspondência AdS/CFT é que eles nos dão um rico dicionário entre operadores das teorias de calibre que vivem na fronteira do espaço e objetos das teorias de cordas no interior do espaço. De acordo com este dicionário, os laços de Wilson, que vivem na 
fronteira do espaço de dez dimensões, são descritos na representação dual de cordas em termos de cordas abertas macroscópicas estendidas no interior do espaço, atracadas na fronteira ao longo do contorno dado, veja Figura 1.1.

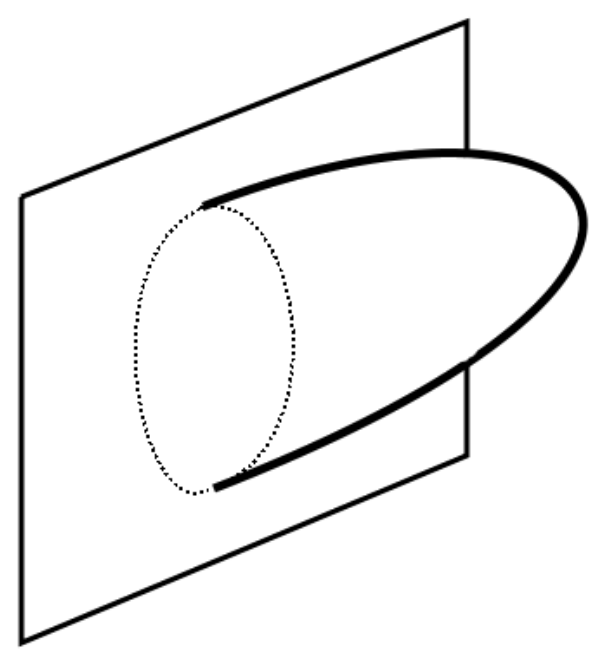

Figura 1.1: Um laço de Wilson supersimétrico na teoria de calibre (o contorno pontilhado na figura) corresponde a uma superfície embutida no interior do espaço e com a borda ao longo do contorno do operador da teoria de calibre.

O valor esperado dos operadores do laço de Wilson são assim dados, na abordagem da teoria de cordas, pela área mínima varrida por esta corda $[13,14]$. Esta área mínima pode ser facilmente calculada e comparada com o resultado da integral do modelo matricial na eq. (1.4), extrapolado no regime de acoplamento forte. Esta comparação nos dá uma concordância perfeita. 


\section{Estrutura da dissertação}

Esta dissertação será estruturada da seguinte forma.

Primeiramente, introduziremos os operadores laços de Wilson em teorias de calibre, construindo-os e desta forma explicitando a sua invariância por calibre. A seguir, calcularemos o valor esperado destes no caso de teorias puramente de calibre abeliana e não abeliana.

Em seguida, daremos argumentos gerais que motivam a correspondência AdS/CFT. Faremos uma revisão da conjectura de Maldacena, utilizando a interpretação física das D-branas como suporte desta. Uma vez estabelecida a conjectura, apresentaremos as características básicas que compõe as duas teorias envolvidas, assim como verificações das simetrias e aspectos do dicionário da correspondência.

Uma vez inseridos no contexto da dualidade, seremos naturalmente levados ao cálculo dos laços de Wilson no contexto da teoria de calibre supersimétrica. Para implementarmos a supersimetria, restringiremos o contorno dos operadores à contornos de linha infinita $\mathrm{e}$ circular. Dando sequência ao cálculo do valor esperado no vácuo desses, implementaremos uma análise perturbativa, o que nos levará a um problema de contagem de uma certa classe de diagramas planares.

Motivados pelo problema de contagem de diagramas planares, o modelo de matrizes é apontado como solução, através de argumentos que faremos seguindo a prova de Pestun, que estabelece a relação entre teorias de calibre supersimétricas e modelo de matrizes. Após uma introdução definindo o modelo, faremos uma revisão das principais técnicas utilizadas neste contexto, o método de aproximação por ponto de sela e o método dos polinômios ortogonais. Tais métodos nos resultarão em valores exatos para os laços de Wilson em questão.

Tendo os resultados de um lado da correspondência, abordaremos os mesmos operadores do ponto de visto do lado da gravidade. A relação dos operadores em tal contexto é estabelecida através do cálculo de superfícies minimais, que são calculadas utilizando a ação clássica da teoria de cordas. Os resultados são obtidos e comparados nos dois lados da dualidade, verificando não trivialmente a correspondência AdS/CFT.

Por fim, apresentaremos conclusões e comentários. 


\section{Capítulo 2}

\section{Laços de Wilson em teorias de calibre}

Como foi mencionado na introdução, os laços de Wilson são operadores que podem ser definidos em qualquer teoria de calibre. Antes de estudar o caso supersimétrico no contexto da correspondência AdS/CFT, nesta seção vamos fazer uma breve revisão destes operadores em um contexto geral de uma teoria de calibre. Começaremos introduzindo ingredientes para fazer a sua construção, como os links de Wilson, e desta forma observar sua invariância de calibre. Em seguida calcularemos o valor esperado destes operadores para teorias abelianas e não abelianas. Para o caso abeliano, através do cálculo deste operador, vamos obter um potencial coulombiano. No caso não abeliano vamos estudar o comportamento do operador em regimes não perturbativos, introduzindo uma rede discretizada, e obter um potencial linear confinante. Neste capítulo vamos seguir de forma muito próxima ao que foi feito em [15].

\subsection{Links de Wilson}

Para construir os laços de Wilson, vamos considerar uma teoria puramente de Yang-Mills, somente com campos de calibre sem conteúdo de matéria, cujo grupo de calibre é $S U(N)$. Além disso, vamos considerar dois pontos do espaço-tempo $x^{\mu}$ e $x^{\mu}+\epsilon^{\mu}$, onde $\epsilon^{\mu}$ é um vetor infinitesimal. Assim, podemos definir o link de Wilson como sendo ${ }^{1}$

$$
W(x+\epsilon, x) \equiv \exp \left[i g \epsilon^{\mu} A_{\mu}(x)\right],
$$

\footnotetext{
${ }^{1}$ Somente neste capítulo introduzimos a costante de acoplamento $g$ da teoria no exponencial.
} 
onde estamos denotando os campos de calibre por $A_{\mu}(x) \equiv A_{\mu}^{a}(x) T^{a}$, sendo que $T^{a}$ são matrizes hermitianas de traço nulo geradoras de $S U(N)$, e $g$ é a constante de acoplamento da teoria considerada.

Este objeto esta associado à fase adquirida pela função de onda de uma partícula carregada percorrendo uma distância infinitesimal na presença do campo de calibre em questão. Uma vez que $\epsilon$ é infinitesimal, podemos fazer uma expansão da conexão de Wilson em potências de $\epsilon$, ou seja,

$$
W(x+\epsilon, x)=1+i g \epsilon^{\mu} A_{\mu}(x)+\mathcal{O}\left(\epsilon^{2}\right) .
$$

Agora, vamos analisar o comportamento deste objeto através de uma transformação de calibre, dado que o campo de calibre transforma-se como

$$
A_{\mu}(x) \rightarrow U(x)\left(A_{\mu}(x)+\frac{i}{g} \partial_{\mu}\right) U^{\dagger}(x)
$$

sendo $U(x)$ matrizes unitarias especiais que dependem do espaço-tempo. Por consequência, o link de Wilson irá transformar-se da forma

$$
W(x+\epsilon, x) \rightarrow 1+i g \epsilon^{\mu} U(x) A_{\mu}(x) U^{\dagger}(x)-\epsilon^{\mu} U(x) \partial_{\mu} U^{\dagger}(x)+\mathcal{O}\left(\epsilon^{2}\right)
$$

Notando que $U U^{\dagger}=1$, e por consequência $\left(\partial_{\mu} U\right) U^{\dagger}=-U\left(\partial_{\mu} U^{\dagger}\right)$, nós temos que (2.3) pode ser escrita como

$$
W(x+\epsilon, x) \rightarrow\left[\left(1+\epsilon^{\mu} \partial_{\mu}\right) U(x)\right] U^{\dagger}(x)+i g \epsilon^{\mu} U(x) A_{\mu}(x) U^{\dagger}(x)+\mathcal{O}\left(\epsilon^{2}\right) .
$$

Podemos ver o primeiro termo como uma expansão em primeira ordem de $U(x)$, ou seja, $U(x+\epsilon)=U(x)+\epsilon^{\mu} \partial_{\mu} U(x)+\mathcal{O}\left(\epsilon^{2}\right)$, enquanto que no segundo termo podemos aproximar, pela mesmo razão, $U(x+\epsilon)$ por $U(x)$, ignorando termos de segunda ordem em $\epsilon$. Assim, o resultado será

$$
W(x+\epsilon, x) \rightarrow U(x+\epsilon)\left(1+i g \epsilon^{\mu} A_{\mu}(x)+\mathcal{O}\left(\epsilon^{2}\right)\right) U^{\dagger}(x) .
$$

Portanto, nós temos que a transformação de calibre do operador será

$$
W(x+\epsilon, x) \rightarrow U(x+\epsilon) W(x+\epsilon, x) U^{\dagger}(x) .
$$




\subsection{Invariância de calibre}

Uma vez obtida a transformação de calibre dos links de Wilson, podemos nos perguntar qual é o comportamento de uma cadeia de links de Wilson. Vamos construir esta a partir de um ponto $x^{\mu}$ seguido por uma sequência de $n$ pontos separados por vetores $\epsilon_{i}^{\mu}$, $i=1,2, \ldots n$, terminando em um ponto $x^{\prime \mu}$. Tais pontos são ordenados formando um caminho no espaço-tempo, sendo que denotaremos este caminho por $P$. Chamaremos de linha de Wilson o seguinte objeto:

$$
W_{P}\left(x^{\prime}, x\right) \equiv W\left(x, x^{\prime}-\epsilon_{n}\right) \ldots W\left(x+\epsilon_{1}+\epsilon_{2}, x+\epsilon_{1}\right) W\left(x+\epsilon_{1}, x\right) .
$$

Utilizando a equação (2.7), podemos ver que este objeto, sob uma transformação de calibre, se transformará como o link de Wilson

$$
W_{P}\left(x^{\prime}, x\right) \rightarrow U\left(x^{\prime}\right) W_{P}\left(x^{\prime}, x\right) U^{\dagger}(x)
$$

uma vez que as matrizes $U$ que aparecem entre os links vão se cancelar mutuamente. Se ao invés de escolhermos pontos distintos de início e fim para o curva $P$ escolhermos um caminho fechado, ou seja $x^{\prime}=x$, iremos obter uma curva fechada no espaço-tempo que denotaremos por $C$.

Finalmente, podemos definir um laço de Wilson como sendo o traço de uma linha de Wilson cujo caminho é fechado. Mais explicitamente

$$
W_{C} \equiv \operatorname{Tr} W_{C}(x, x)=\operatorname{Tr} \mathcal{P} \exp \left(i g \oint_{C} A_{\mu} d x^{\mu}\right)
$$

onde aqui tomamos o limite de cada $\epsilon_{i}$ indo a zero. Por conta disso, tivemos que introduzir o simbolo $\mathcal{P}$, denotando que a integral deve ser feita de forma ordenada, ou seja

$$
\mathcal{P} \exp \int_{\tau_{0}}^{\tau} d \tau^{\prime} f\left(\tau^{\prime}\right)=1+\int_{\tau_{0}}^{\tau} d \tau^{\prime} f\left(\tau^{\prime}\right)+\int_{\tau_{0}}^{\tau} d \tau^{\prime} \int_{\tau^{\prime}}^{\tau} d \tau^{\prime \prime} f\left(\tau^{\prime}\right) f\left(\tau^{\prime \prime}\right)+\ldots,
$$

onde o fator de $1 / n$ ! da expansão de Taylor foi cancelado escolhendo um ordenamento particular dos parâmetros (por exemplo, $\tau_{0} \leq \tau^{\prime} \leq \tau^{\prime \prime} \leq \ldots \leq \tau$ ).

Daqui podemos ver duas importantes propriedades: Este operador é não localizado, não possui dependência de apenas um único ponto do espaço-tempo, sendo suportado pela curva $C$, e, além disso, é invariante por transformações de calibre

$$
W_{C} \longrightarrow W_{C}
$$


sendo que para chegar neste resultado utilizamos a propriedade cíclica do traço e a unitariedade das matrizes $U$.

\subsection{Caso abeliano}

Uma vez definido o operador, vamos calcular o valor esperado no vácuo (VEV) deste no caso mais simples de uma teoria de calibre, no contexto do grupo $U(1)$ sem campos carregados. Neste caso de um grupo abeliano, o cálculo é simples e pode ser feito de maneira analítica. A ação desta teoria será:

$$
S=\int d^{4} x \mathcal{L}\left(A_{\mu}\right)=\int d^{4} x \frac{1}{4} F^{\mu \nu} F_{\mu \nu}
$$

onde temos que $F_{\mu \nu}=\partial_{\mu} A_{\mu}-\partial_{\nu} A_{\mu}$. Fazendo uma rotação de Wick na direção temporal $(t \rightarrow i t)$, o VEV do laço de Wilson será dado por

$$
\left\langle 0\left|W_{C}\right| 0\right\rangle \equiv\left\langle W_{C}\right\rangle=\int \mathcal{D} A e^{i g \oint_{C} d x_{\mu} A^{\mu}} e^{-S}
$$

Aqui podemos interpretar formalmente $i g \oint_{C} d x_{\mu}$ como uma corrente externa $J_{\mu}(x)$ com suporte compacto sobre $C$, tal que $\int d^{4} x A^{\mu} J_{\mu}(x)=i g \oint_{C} d x_{\mu} A^{\mu}$. Desta forma, vemos que a conta se reduz ao cálculo da função geradora da função de Green do campo eletromagnético, que, como pode ser visto no Apêndice I, nos resulta em

$$
\left\langle W_{C}\right\rangle=\exp \left[-\frac{1}{2} g^{2} \oint_{C} d x_{\mu} \oint_{C} d y_{\nu} \Delta_{\mu \nu}(x-y)\right],
$$

sendo $\Delta_{\mu \nu}(x-y)$ o propagador do fóton em termos das posições no espaço-tempo euclidiano. Temos que este propagador pode ser obtido fazendo a transformação do espaço de momentos do propagador usual, no calibre de Feynman, para o espaço de posições, ou seja,

$$
\Delta_{\mu \nu}(x-y)=\delta_{\mu \nu} \int \frac{d^{4} k}{(2 \pi)^{4}} \frac{e^{i k \cdot(x-y)}}{k^{2}}=\frac{\delta_{\mu \nu}}{4 \pi^{2}(x-y)^{2}} .
$$

Utilizando (2.16) na equação (2.15), podemos obter

$$
\left\langle W_{C}\right\rangle=\exp \left[-\frac{1}{2} g^{2} \oint_{C} d x^{\mu} \oint_{C} d y^{\nu} \frac{\delta_{\mu \nu}}{4 \pi^{2}(x-y)^{2}}\right] .
$$

Notemos que nesta conta o $\delta_{\mu \nu}$ efetuará o produto escalar euclidiano de $d x^{\mu} \operatorname{com} d y^{\nu}$. Com isto em mente, vamos fazer o cálculo no caso de um contorno retangular de lados $D$ 
na direção espacial e $T$ na direção temporal, com $a \ll D \ll T$, sendo $a$ o corte quando $x^{\mu} \rightarrow y^{\mu}$. Podemos dividir o cálculo em casos, dependendo de quais lados do circuito estamos fazendo o produto escalar. Caso $x^{\mu}$ e $y^{\nu}$ estejam do mesmo lado do circuito $C$, o produto escalar resultará em $d x \cdot d y=d x d y$, e nós teremos que a integral se reduzirá à

$$
\int_{0}^{Q} \int_{0}^{Q} \frac{d x d y}{(x-y)^{2}}=\lim _{a \rightarrow 0} \int_{0}^{Q} d y\left[\int_{-a}^{y-a} \frac{d x}{(x-y)^{2}}+\int_{y+a}^{Q+a} \frac{d x}{(x-y)^{2}}\right] \simeq 2 Q / a-2 \ln (Q / a)
$$

sendo $Q$ o comprimento do lado em que se encontram $x$ e $y(D$ ou $T)$. No caso em que $x^{\mu}$ e $y^{\nu}$ são ortogonais nós temos que $d x \cdot d y=0$. Porém, quando estes estão em lados opostos do retângulo, de comprimento $D$, nós teremos que a integral será proporcional à $D^{2} / T^{2}$, podendo ser desprezado, uma vez que $D \ll T$. Finalmente, se $x$ está em $T$ e $y$ no respectivo oposto $(d x \cdot d y=-d x d y)$, nós temos

$$
\int_{0}^{T} \int_{0}^{T} \frac{d x d y}{(x-y)^{2}+D^{2}} \simeq \pi T / D-2 \ln (T / D)-2 .
$$

Somando todas estas contribuições e tomando o limite de $T$ grande obtemos que

$$
\left\langle W_{C}\right\rangle=\exp \left[-\frac{1}{2} \alpha \oint_{C} \oint_{C} \frac{d x \cdot d y}{(x-y)^{2}}\right]=\exp \left[-\left(\frac{2 \alpha}{\pi a}-\frac{\alpha}{D}\right) T\right]
$$

onde $\alpha=g^{2} / 4 \pi$. Tal resultado pode ser interpretado fisicamente utilizando o fato de que $\left\langle W_{C}\right\rangle=\exp (-T V(D))$, escolhendo um contorno retangular cujos lados são $D$ e $T$, e para $T$ indo a infinito, onde $V(D)$ é o potencial entre duas partículas carregadas colocadas a uma distância $D$ uma da outra. Desta forma podemos interpretar o termo $2 \alpha / \pi a$ como uma divergência devido à abordagem pontual do sistema, enquanto o outro termo, $\alpha / D$, é de fato o potencial coulombiano.

\subsection{Caso não abeliano}

No caso não abeliano podemos fazer um cálculo semelhante no regime perturbativo, para a constante de acoplamento pequena. Fazendo a expansão da exponencial do operador laço de Wilson obtemos um resultado semelhante ao caso abeliano, para a primeira contribuição não trivial

$$
\left\langle W_{C}\right\rangle=\operatorname{Tr} \mathcal{P}\left[1-\frac{1}{2} g^{2} T^{a} T^{a} \oint_{C} d x_{\mu} \oint_{C} d y_{\nu} \Delta_{\mu \nu}(x-y)+\mathcal{O}\left(g^{4}\right)\right]
$$




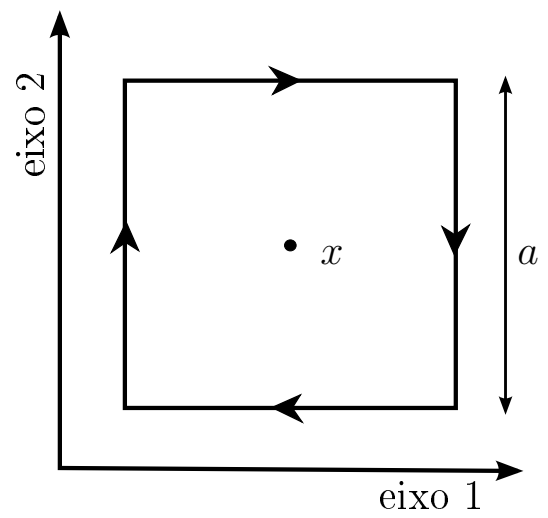

Figura 2.1: Uma plaqueta centrada em $x$ e de lado $a$, com as direções 1 e 2 explicitadas.

Sabendo que $T^{a} T^{a}$ é igual ao operador de Casimir quadrático, com o valor $C(R)$ dependente da representação $R$, vemos que as contas são as mesmas do caso abeliano, porém com a substituição de $g^{2} \rightarrow g^{2} C(R)$.

Para a análise no regime não perturbativo, nós vamos definir uma ação na rede. Para isso, vamos considerar uma rede que fraciona o espaço-tempo euclidianizado em pequenos hipercubos de largura $a$. Será útil considerarmos vetores $v_{n}$ nas direções $n$, de comprimento $a$, e escolher o ponto $x$ como sendo o centro do hipercubo. Dada esta partição do espaço-tempo, a menor linha de Wilson que podemos construir será um link de Wilson de comprimento elementar $a$. Vamos definir uma plaqueta como sendo um quadrado elementar orientado formado por quatro links de Wilson, ver Figura 2.1, ou seja,

$$
W_{\text {plaq }} \equiv \operatorname{Tr} e^{-i g a A_{2}\left(x-v_{1} / 2\right)} e^{-i g a A_{1}\left(x-v_{2} / 2\right)} e^{+i g a A_{2}\left(x-v_{1} / 2\right)} e^{+i g a A_{1}\left(x-v_{1} 2 / 2\right)}
$$

onde $A_{1}$ e $A_{2}$ são respectivamente as componentes do campo de calibre nas direções 1 e 2. Tratando os campos de calibre como funções suaves e expandindo estas em torno de $x$, podemos obter

$$
\begin{aligned}
W_{\text {plaq }}= & \operatorname{Tr} e^{-i g a A_{2}(x)+i g a^{2} \partial_{1} A_{2}(x) / 2+\ldots} e^{-i g a A_{1}(x)+i g a^{2} \partial_{2} A_{1}(x) / 2+\ldots} \\
& \times e^{+i g a A_{2}(x)+i g a^{2} \partial_{1} A_{2}(x) / 2+\ldots} e^{+i g a A_{1}(x)+i g a^{2} \partial_{2} A_{1}(x) / 2+\ldots}
\end{aligned}
$$

Utilizando a identidade $e^{A} e^{B}=e^{A+B+[A, B] / 2 \cdots}$, nós podemos combinar a primeira com a segunda exponencial da primeira linha, e a terceira com a quarta, da segunda linha, e 
assim obter

$$
W_{\text {plaq }}=\operatorname{Tr} e^{i g a^{2}\left(\partial_{1} A_{2}-\partial_{2} A_{1}-i g\left[A_{1}, A_{2}\right]\right)+\ldots}
$$

Disso podemos obter que

$$
W_{\text {plaq }}+W_{-p l a q}=2 N-g^{2} a^{4} \operatorname{Tr} F_{12}^{2}
$$

sendo que $F_{12}=\partial_{1} A_{2}-\partial_{2} A_{1}-i g\left[A_{1}, A_{2}\right]$ e $W_{-p l a q}$ é $W_{p l a q}$ com a orientação inversa.

Assim vemos que podemos definir a ação da teoria em termos das plaquetas como sendo

$$
S=-\frac{1}{2 g^{2}} \sum_{\text {plaq }} W_{\text {plaq }}
$$

onde a soma é feita sobre todas as plaquetas onde o campo está definido e nas duas possíveis orientações. Considerando $U$ como sendo a matriz associada a um link orientado, e assim $U^{\dagger}$ o mesmo link no sentido oposto, nós podemos escrever a integral de trajetória em termos destes links

$$
Z=\int \mathcal{D} U e^{-S}
$$

sendo que

$$
\mathcal{D} U=\prod_{\text {link }} d U_{\text {link }}
$$

onde $d U$ é a medida de Haar para matrizes especiais unitárias. Esta medida é normalizada tal que $\int d U=1$, e possui as seguintes propriedades:

$$
\begin{aligned}
\int d U U_{i j} & =0 \\
\int d U U_{i j} U_{k l} & =0 \\
\int d U U_{i j} U_{k l}^{*} & =\frac{1}{N} \delta_{i k} \delta_{j l} .
\end{aligned}
$$

A última identidade implica que, ao fazer a integração, dois links de orientações opostas vão se cancelar. Desta forma, quando tivermos duas plaquetas adjacentes orientadas, no mesmo sentido, elas vão se mesclar formando um único retângulo maior, como na Figura 2.2. Para o regime de acoplamento forte, nós teremos $1 / g^{2}$ assumindo valores 


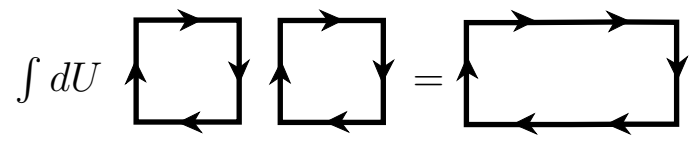

Figura 2.2: Ilustração da terceira propriedade em (2.29).

pequenos, e assim nós podemos expandir o operador na forma

$$
\begin{aligned}
\left\langle W_{C}\right\rangle & =\frac{1}{Z} \int \mathcal{D} U W_{C} \exp \left\{-\frac{1}{2 g^{2}} \sum_{\text {plaq }} W_{\text {plaq }}\right\} \\
& =\frac{1}{Z} \int \mathcal{D} U W_{C}\left\{1-\frac{1}{2 g^{2}} \sum_{\text {plaq }} W_{\text {plaq }}+\frac{1}{2 !}\left(\frac{1}{2 g^{2}}\right)^{2} \sum_{\text {plaq }} \sum_{\text {plaq }} W_{\text {plaq }} W_{\text {plaq }}+\ldots\right\} .
\end{aligned}
$$

Para resolver este cálculo, vamos usar as propriedades listadas em (2.29). Lembrando que $W_{C}$ e $W_{\text {plaq }}$ são produtos de links $U$, podemos utilizar a propriedade de ortogonalidade dos Us. Assim, vemos que a contribuição de mais baixa ordem não nula será dada pelo termo composto pelo produto do conjunto de plaquetas que preenchem a área circunscrita pelo contorno $C$. Todos os links de linhas internas, devido às plaquetas, irão se cancelar por haver um link de orientação oposta dentro do produto de links. Desta forma, temos que a primeira contribuição não nula será

$$
\left\langle W_{C}\right\rangle \sim\left(\frac{1}{g^{2}}\right)^{N_{p}}
$$

Sendo $N_{p}=A(C) / a^{2}$, onde $A(C)$ é a área interna ao contorno $C$, ou seja, $N_{p}$ é igual ao número de plaquetas utilizadas para cobrir a área $A(C)$. Tal dependência do laço de Wilson com a área é chamada de lei da área. Podemos expressar isso da seguinte forma

$$
\left\langle W_{C}\right\rangle \sim\left(g^{2}\right)^{-A(C) / a^{2}}=\exp \left[-\left(T D \ln g^{2}\right) / a^{2}\right]
$$

onde usamos um contorno $C$ como foi descrito no exemplo abeliano. Assim, comparando $\operatorname{com}\left\langle W_{C}\right\rangle \sim \exp (-E T)$ nós podemos concluir que

$$
E(D)=K D
$$


onde

$$
K=\frac{\ln g^{2}}{a^{2}}
$$

é chamada de tensão da corda. Portanto, nós vemos que a energia do sistema depende linearmente da distância. Podemos imaginar partículas carregadas se propagando ao longo dos lados do laço de Wilson, então para afastarmos as duas partículas a uma distância infinita nós teremos que utilizar uma quantidade infinita de energia, assim dizemos que as cargas estão confinadas. Apesar deste fato parecer razoável, e até mesmo desejável, uma vez que é condizente com os resultados da QCD, este resultado não é definitivo. Uma vez que para discretizar o espaço-tempo introduzimos um parâmetro de corte $a$, precisamos de alguma forma voltar ao limite do contínuo e obter um resultado independente deste parâmetro. Também, ao olharmos para o limite de $g$ indo a zero esperamos que o coeficiente $K$ em algum momento se anule para um valor de $g$ diferente de zero. Acontecendo isto, nós poderemos ter uma transição de fase para um limite não confinado. Entretanto, tal procedimento para a obtenção consistente do limite do contínuo ainda não foi desenvolvido e demonstrar confinamento de cor na QCD permanece um problema em aberto. 


\section{Capítulo 3}

\section{Revisão da correspondência AdS/CFT}

\subsection{Considerações gerais}

Nesta seção, iremos apresentar alguns argumentos gerais que motivam a correspondência entre uma teoria de calibre e uma teoria de gravidade quântica. Apesar da correspondência AdS/CFT, primeiramente proposta por J. Maldacena [1], ser o caso mais bem entendido, alguns argumentos são mais gerais e dão suporte aos outros casos de dualidade calibre/gravidade. Aqui seguiremos a mesma linha de raciocínio da revisão [7].

De forma generalizada, a proposta da correspondência é de que certas teorias quânticas de campos são, de forma não trivial, duais ou equivalentes a teorias de gravidade quântica.

Mesmo sendo desconhecido o entendimento completo do que deva ser uma teoria quântica da gravidade, o que esperamos de tal é que seja uma teoria quântica cuja métrica possua uma dinâmica, em outras palavras, exista uma partícula não massiva propagante cujo spin seja 2. Tal partícula, com estas características, é conhecida na literatura como gráviton. Porém, também é um fato conhecido na literatura que tais teorias quânticas de campos, que possuem um gráviton, acabam por deparar-se com o teorema de WeinbergWitten [16], cuja afirmação é que partículas não massivas cujo spin seja maior 1 não podem existir em uma teoria quântica relativística que possua um tensor covariante de stress $T^{\mu \nu}$ conservado. Basicamente, como consequência deste teorema, tais teorias não podem pos-

suir grávitons propagantes, uma vez que $p^{\mu}=\int d \vec{x} T^{0 \mu}=0$. Além desta dificuldade, outra discrepância que logo nota-se é que teorias de gravidade não possuem observáveis locais, 
uma vez que não faz sentido definir sistemas de coordenadas locais de forma invariante, e por outro lado, sabemos que teorias quânticas de campos possuem tais operadores, o que gera uma divergência entre o número de observáveis.

Devido a estas dificuldades, durante muito tempo uma união ou relação entre estas duas teorias foi difícil de ser encontrada, mas ao explorar uma brecha destes argumentos pôde-se concretizar esta relação. O ponto que pôde ser explorado é o fato de que as duas teorias em questão não precisam viver no mesmo espaço. Uma vez colocada cada teoria em um espaço distinto, os argumentos anteriores, que implicavam em incompatibilidade, deixam de valer.

Apesar de sabermos que precisamos definir cada teoria em espaços distintos, ainda não é claro qual deve ser a relação entre eles para obtermos uma dualidade. Para ajudar na escolha dos espaços, devemos olhar para dois fatos, um de cada teoria, que nos dá intuição destas escolhas.

- O princípio holográfico. Sabemos que em uma teoria de gravidade a máxima entropia que podemos obter dado um volume $V$ finito é igual à entropia de um buraco negro com estas respectivas proporções

$$
S_{B H}=\frac{A}{4 G_{N}},
$$

sendo que $A$ refere-se à área que encerra o volume $V$ e $G_{N}$ é a costante de Newton. Este fato, da entropia escalar com a área da região considerada e não com o volume, destoa do usual cálculo de entropia no caso de teorias quânticas de campos, que, em linhas gerais, é proporcional ao volume do sistema considerado. Esta observação nos indica que, se queremos que as entropias de dois sistemas que sejam equivalente em ambas as descrições coincidam, nós temos que ter a teoria de gravidade vivendo em um espaço cuja dimensão seja maior do que aquela proposta para a teoria de campos.

- "Geometrização" da escala de energia. Neste caso olhamos para o grupo de renormalização da teoria, onde organizamos as teorias em termos da sua dependência com um parâmetro de escala $r$, assim vendo cada teoria como sendo distinta uma da 
outra para cada valor do parâmetro fixo. Por exemplo, a forma como a constante de acoplamento $g$ depende deste parâmetro é dada pela função beta da teoria

$$
\beta(g(r))=r \partial_{r} g(r)
$$

Esta equação expressa uma dependência 'local' da teoria com relação ao parâmetro $r$, sendo que aqui estamos falando de localidade de forma análoga a que usamos para designar dependências de um ponto do espaço-tempo. Assim podemos olhar para esta equação como sendo a descrição da dependência local da teoria com relação a um parâmetro $r$, que fomos impelidos a interpretar como uma dimensão extra espacial, dando uma interpretação física para a dimensão extra sugerida pelo princípio holográfico.

Portanto estas observações nos dão indícios de que podemos relacionar uma teoria quântica da gravidade com uma teoria quântica de campos, sendo que estas precisam viver em espaços distintos e relacionados pelos argumentos dados acima, podendo ser resumidos da seguinte forma: A teoria gravitacional deve viver em um espaço cuja dimensão seja maior do que a do espaço onde a teoria de calibre é definida, para que assim as entropias possam ser identificadas, sendo que a dimensão extra existente na teoria de gravidade pode ser identificada com a escala de energia da teoria de calibre.

Uma observação direta que podemos fazer, assumindo a existência de tal dualidade, é que aspectos quânticos de uma teoria de gravidade não podem estar relacionados com a teoria quântica de campos em regime perturbativo, uma vez que nestes regimes não observamos manifestações de gravidade quântica. Observado isto, somos levados a concluir que os regimes acessíveis perturbativamente de cada teoria devem estar relacionados com os regimes não perturbativos da outra, ou seja, uma relação de forte/fraco acoplamento.

Esta relação inversa entre os regimes perturbativos é um dos pontos que torna a correspondência tão interessante. Uma vez que é possível traduzir um problema não perturbativo de um lado da dualidade em outro problema perturbativo, teremos uma ferramenta para atacar problemas não perturbativos, que são de extrema importância em muitos sistemas físicos. Ao mesmo tempo, esta falta de intersecção entre os regimes perturbativos faz com que a correspondência seja difícil de ser verificada, uma vez que, em geral, não temos 
acesso às duas descrições para os mesmo objetos em ambos os domínios de validade das teorias.

Porém, em alguns casos, nós podemos fazer os cálculos de alguns observáveis em ambas as descrições. Como veremos, um destes observáveis são os operadores laços de Wilson supersimétricos, que, no contexto da conjectura de Maldacena, são objetos possíveis de serem calculados em ambas as descrições. Mais do que isso, verificaremos que eles podem ser calculados em regimes não perturbativos em uma das descrições. Por diversas razões, tais objetos são de grande interesse, sendo principalmente estudados por nos permitir fazer algumas checagens não triviais da validade da dualidade, o que se torna de grande importância uma vez que não temos uma demonstração formal da correspondência.

\subsubsection{Exemplo da correspondência AdS/CFT}

Como exemplo mais simples que podemos imaginar para ilustrar o que foi dito, podemos analisar uma teoria quântica de campos invariante por escala. Neste caso, isso se traduz como $\beta(g(r))=0$, ou seja, a teoria não depende da variável de escala $r$. Para ver como isto se reflete na métrica do espaço tempo, vamos olhar para uma transformação de escala que se realiza na forma

$$
x^{\mu} \rightarrow \lambda x^{\mu}, \quad r \rightarrow \frac{r}{\lambda}
$$

sendo $\lambda$ um fator de escala e $\mu$ índices do espaço-tempo $(\mu=0, \ldots, d-1)$. A transformação de $r$ é dada pelo fato da energia possuir dimensão natural inversa da dimensão de espaço (em unidades naturais, $\hbar=c=1$, que serão utilizadas durante todo o trabalho). Levando estas transformações em conta, e o fato de que queremos uma métrica que seja invariante por transformações de Poincaré, uma métrica invariante por escala em $d+1$ dimensões pode ser realizada como

$$
d s^{2}=\frac{r^{2}}{L^{2}} \eta_{\mu \nu} d x^{\mu} d x \nu+\frac{L^{2}}{r^{2}} d r^{2}
$$

que é reconhecida como a métrica AdS em $d+1$ dimensões com raio $L$.

Uma das propriedades desta métrica é ser solução das equações de movimento de uma ação da forma

$$
S=\frac{1}{16 \pi G_{N}} \int d^{d+1} x \sqrt{-g}(R-2 \Lambda+\ldots)
$$


onde $-2 \Lambda=\frac{d(d-1)}{L^{2}}$ e as reticências são termos com derivadas mais altas que podemos ignorar.

Uma forma de se obter uma ação desta forma é através do espectro da teoria de cordas a baixas energias, ou seja, quando $1 / L^{2} \ll 1 / \alpha^{\prime} \sim 1 / \ell_{s}^{2}$, onde $\alpha^{\prime}$ é o parâmetro de Regge e $\ell_{s}$ é a escala de comprimento da corda, sendo estes relacionados por $\ell_{s}^{2}=2 \alpha^{\prime}$. Além dos termos explicitados acima, com o espectro da teoria de cordas nós podemos obter correções para a gravidade clássica e completar a ação acima.

Para que de fato possamos considerar esta ação clássica, nós devemos ter que a razão $\ell_{P} / L$ seja pequena, onde $\ell_{P}$ é a escala de Planck, que nos diz em qual escala os efeitos da gravidade quântica são importantes. Esta relação, $\ell_{P} / L$, funciona como " $\hbar$ efetivo" da teoria, ou seja, quando esta relação é pequena nós podemos utilizar uma teoria de gravidade clássica.

Motivados por esta análise, somos impelidos a relacionar uma classe de teorias quânticas de campo invariantes por escala em $d$ dimensões com uma teoria de gravidade assintoticamente AdS em $d+1$ dimensões. Aqui, tomamos o cuidado de somente pensar em uma gravidade assintoticamente AdS, uma vez que estamos tratando de uma teoria de gravidade, ou seja, estamos lidando com um espaço-tempo dinâmico, onde temos que fixar as condições de contorno para fazer a integração na métrica. De fato, as condições de contorno aqui são relevantes por conta do fato de que estados com trajetória do tipo luz podem alcançar, em tempo finito, a fronteira de uma métrica AdS. Veremos isso com mais detalhes na Seção 3.4.

\subsubsection{Contando os graus de liberdade}

Outra forma de conferir a estrutura de raciocínio que tivemos até aqui é verificando o princípio holográfico. Para isto, vamos fazer a contagem dos graus de liberdade de cada teoria.

No caso da teoria quântica de campos, vamos fazer uma contagem utilizando uma rede discreta. Para isso, precisamos definir um parâmetro de corte UV e IV, que denotaremos por $\delta$ e $R$ como sendo, respectivamente, dimensões de espaço para cada parâmetro de corte, como mostra a Figura 3.1 abaixo. 


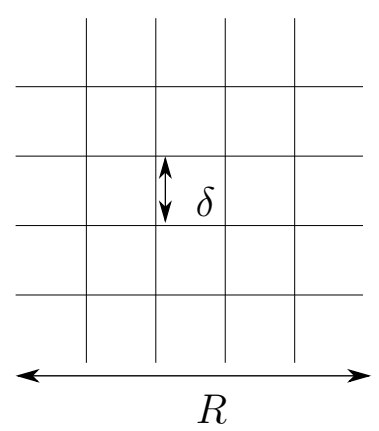

Figura 3.1: Rede de tamanho $R$, onde introduzimos um corte UV, $\delta$, para regularizar o número de graus de liberdade na TQC.

Por razões que ficaram claras na Seção 3.2, vamos definir o número de graus de liberdade por sítio da rede como sendo $N^{2}$. Portanto, o número de graus de liberdade de um sistema de tamanho $R$ será

$$
\left(\frac{R}{\delta}\right)^{d-1} N^{2}
$$

Para o lado da teoria de gravidade, sabemos que o número de graus de liberdade está relacionado com a área delimitada pelo volume deste sistema. Para facilitar a contagem, será útil fazermos a transformação de coordenadas $z=L^{2} / r$, o que nos dá $\sqrt{-g}=(L / z)^{d-1}$ (para um tempo constante). Assim, devemos calcular a área da borda de AdS

$$
A_{\partial A d S}=\int d^{d-1} x\left(\frac{L}{z}\right)^{d-1} .
$$

Uma dificuldade que encontramos neste cálculo é que a métrica é divergente em $z \rightarrow$ 0, onde está localizada a fronteira. Para superar esta dificuldade, podemos utilizar um corte selecionando $z=\delta$ e depois tomando $\delta$ indo a zero, como pode ser visto abaixo na Figura 3.2. Feitas estas observações, temos que

$$
A_{\partial A d S}=\left.\int_{0}^{R} d^{d-1} x\left(\frac{L}{z}\right)^{d-1}\right|_{z=\delta}=\left(\frac{R L}{\delta}\right)^{d-1} .
$$

Encontramos então a mesma dependência nos cortes $\delta$ e $R$ de (3.6).

Se queremos que em ambos os casos tenhamos o mesmo número de graus de liberdade, temos que identificar

$$
\frac{L^{d-1}}{G_{N}}=\left(\frac{L}{\ell_{P}}\right)^{d-1} \sim N^{2}
$$




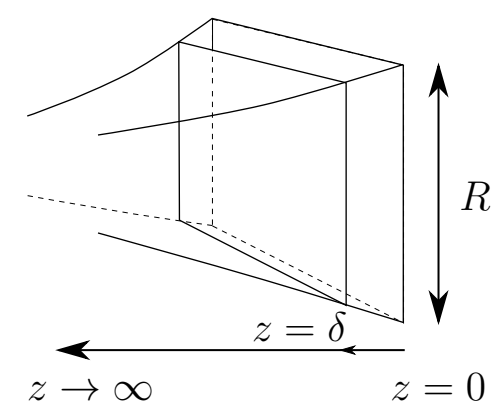

Figura 3.2: Regularização do número de graus de liberdade no interior da AdS.

Comparando com a condição para que a gravidade seja clássica $\ell_{P} / L \gg 1$, vemos que podemos identificar o regime onde a gravidade é clássica com o limite de muitos graus de libertade por sítio da rede, $N^{2} \gg 1$. Na Seção 3.2, este limite, de " $N$ grande", será interpretado em termos de quantidades físicas relacionando a teoria quântica de campos com a teoria de cordas.

Para entender melhor o significado do número $N^{2}$, que introduzimos como sendo o número de graus de liberdade associados a cada sítio da rede, a seguir vamos rever o trabalho de 't Hooft [17]. Através deste trabalho foi possível estabelecer, mais objetivamente, uma forma de realizar a dualidade calibre/gravidade, mas ainda não tão concretamente quanto a proposta de Maldacena.

\subsubsection{O limite de 't Hooft}

Aqui, seguindo o trabalho de [17], vamos buscar uma expansão perturbativa da função de partição de uma teoria de calibre explicitando o número de cores $N$. Em seguida, vamos observar os efeitos de tomarmos o limite para $N$ grande, o que irá implicar numa relação entre diagramas planares e não planares com a expansão em genus da teoria de cordas.

Primeiramente, para estudarmos o limite de 't Hooft, a expansão em $N$, vamos considerar uma teoria de calibre com constante de acoplamento $g_{Y M}$ e cujo grupo seja $S U(N)$. A Lagrangiana, em linhas gerais, pode ser escrita como

$$
\mathcal{L}=\frac{1}{g_{Y M}^{2}} \operatorname{Tr}\left(\left(\partial_{\mu} \Phi_{A}\right)^{2}+\Phi_{A}^{2}+\Phi_{A}^{3}+\ldots\right),
$$


onde estamos denotando por $\Phi_{A}$ qualquer campo na representação adjunta, sendo que o índice $A$ pode representar qualquer conjunto de índices de Lorentz. Porém esses índices não serão relevantes na análise que segue e, portanto, serão omitidos. Podemos explicitar os índices fundamentais e anti-fundamentais desta representação escrevendo $\Phi_{a}^{b}$, onde $a=1, \ldots, \bar{N}$ e $b=1, \ldots, N$ indicam índices anti-fundamentais e fundamentais respectivamente. Esta notação, explicitando os índices fundamentais e anti-fundamentais, será útil para a notação de linha dupla que iremos introduzir a seguir.

Para obter uma expansão no número de cores, 't Hooft introduziu a notação de linha dupla, que consiste em associar linhas orientadas para os índices de cores, $a$ e $b$. A regra é associar linhas com orientações opostas para índices fundamentais e anti-fundamentais, e desta forma teremos linhas duplas paralelas com sentidos opostos para representações adjuntas. Por exemplo, podemos representar o propagador da teoria como na Figura 3.3, enquanto que os vértices, proporcionais à $g_{Y M}^{-2}$, podem ser desenhados da forma apresentada na Figura 3.4.

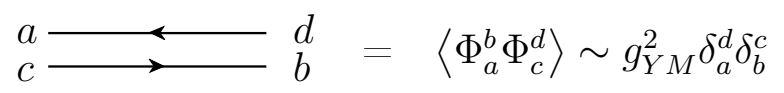

Figura 3.3: Propagador da teoria em notação de linha dupla.

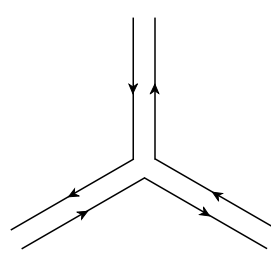

$$
\sim \frac{1}{g_{Y M}^{2}}
$$

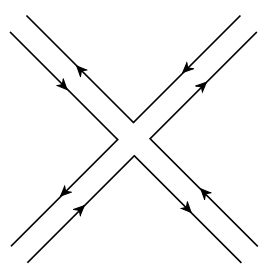

$$
\sim \frac{1}{g_{Y M}^{2}}
$$

Figura 3.4: Vértice triplo (a) e quadruplo (b) em notação de linhas duplas, proporcionais à $g_{Y M}^{-2}$. 
Para um diagrama qualquer, nós teremos que a sua contribuição será proporcional à

$$
\left(g_{Y M}^{2}\right)^{P-V} N^{L} \equiv \lambda^{P-V} N^{L-P+V}
$$

onde denotamos por $P$ o número de propagadores, $V$ o número de vértices, $L$ o número de Loops, e $\lambda$ é o acoplamento de 't Hooft definido como ${ }^{1}$

$$
\lambda \equiv g_{Y M}^{2} N
$$

Alguns exemplos de diagramas do tipo vácuo-para-vácuo, com suas respectivas contribuições, estão ilustradas na Figura 3.5. Notemos que cada diagrama, descrito nesta notação de linha dupla, pode ser desenhado sobre uma superfície de Riemann com o número de Euler igual a $\chi=V-P+L=2-2 h$, sendo $h$ o genus da superfície, como pode ser visto na Figura 3.6.
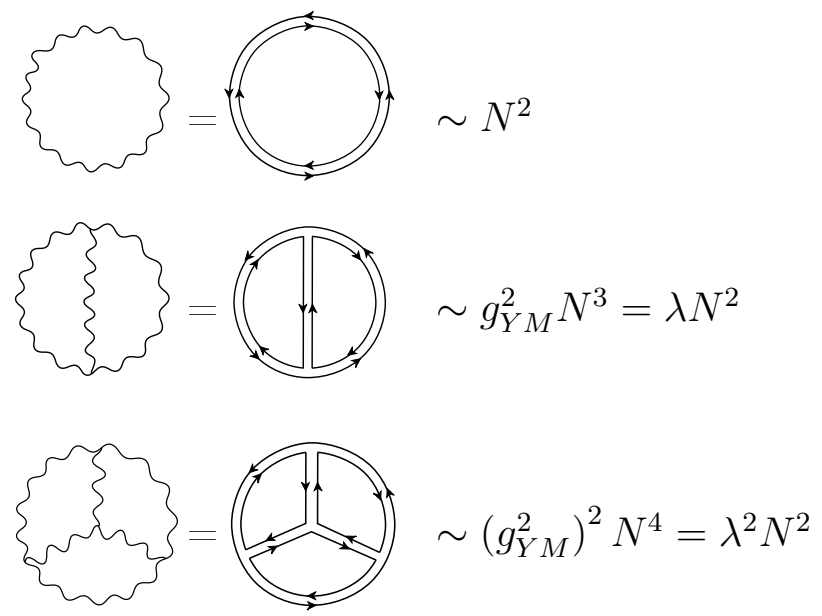

Figura 3.5: Exemplos de diagramas em notação de linhas duplas com suas respectivas contribuições.

É possível escrevermos a equação (3.11) em termos do genus, e portanto escrever a própria expansão da função de partição em termos de uma soma dupla

$$
\ln Z=\sum_{h=0}^{\infty} N^{2-2 h} \sum_{n=0}^{\infty} c_{n}^{(h)} \lambda^{n},
$$

\footnotetext{
${ }^{1}$ Algumas vezes encontrado na literatura com um fator de $2 \pi$ ou $4 \pi$.
} 


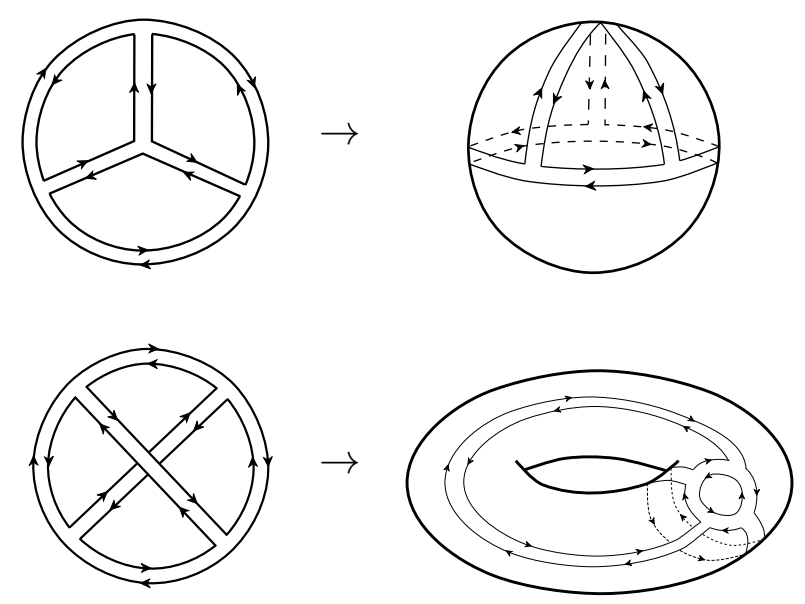

Figura 3.6: Diagramas em notação de linha dupla com suas respectivas superfícies de Riemann.

sendo que $c_{n}^{(h)}$ é um coeficiente que codifica a contribuição dos diagramas de Feynman com genus $h$. Daqui, podemos observar que para o limite de $N$ grande $\operatorname{com} \lambda$ fixo, chamado de limite de 't Hooft, ou limite planar, os diagramas de $h=0$, chamados de diagrams planares (por que podem ser desenhados em um plano sem cruzar as linhas), vão ser dominantes nesta soma.

Até este momento, as teorias abordadas tratavam-se apenas de teorias puramente de calibre, sem campos de matéria, porém a implementação de campos de matéria é feita de maneira simples. Introduzindo campos de matéria, nós iremos introduzir objetos que possuem um único índice (fundamental ou anti-fundamental), o que implicará em diagramas com uma única linha associada a estes campos. Estes diagramas resultantes são contemplados pela associação às superfícies de Riemann com bordas, ver Figura 3.7. Porém, estes diagramas fermiônicos serão suprimidos por diagramas cujas respectivas superfícies são de mesmo genus mas sem bordas.

Voltando a olhar para a soma (3.13), com as superfícies de Riemann associadas em mente, a ligação com a teoria de cordas emerge. Notemos que a primeira soma assemelhase à expansão em genus da teoria de cordas, Figura 3.8, sendo que nesta expansão temos 


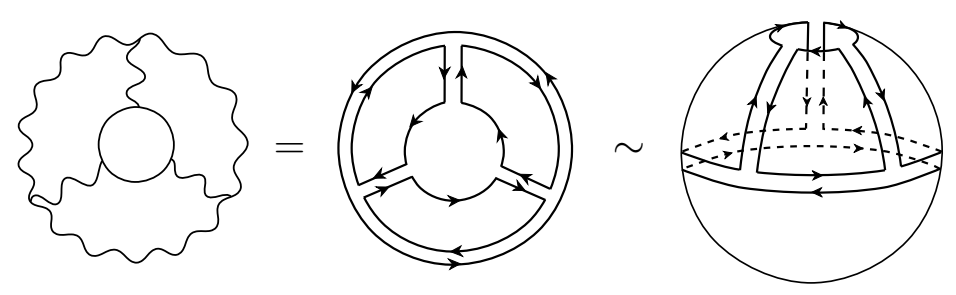

Figura 3.7: Diagramas em notação de linha dupla para férmions com sua respectivas superfícies de Riemann.

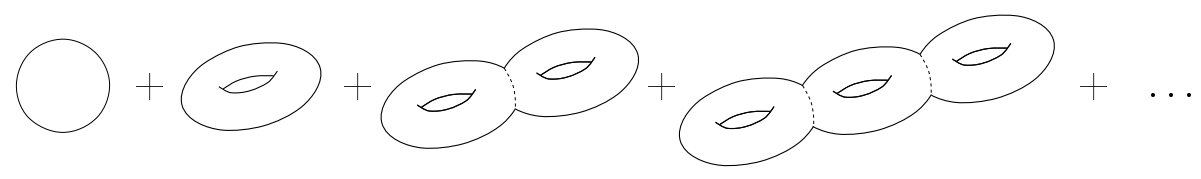

Figura 3.8: Expansão em genus na teoria de cordas.

que os diagramas são suprimidos por fatores da forma $g_{s}^{2 h-2}$, onde $g_{s}$ é o acoplamento das cordas e identificando $h$ com o genus da folha-mundo. A segunda soma que aparece é por sua vez identificada com a expansão em $\alpha^{\prime}$. Observados estes fatos, é natural pensar que, de alguma forma, uma teoria de calibre pode estar relacionada com uma teoria de cordas, cuja constante de acoplamento deva ser algo da forma

$$
g_{s}=\frac{1}{N}
$$

Levada esta relação adiante, podemos inferir uma possível associação entre o limite planar da teoria de campos com o limite fracamente acoplado da teoria de cordas. Da mesma forma, podemos pensar em associar gráficos contendo matéria ao setor de cordas abertas da teoria de cordas.

Até este ponto, temos indícios de relações entre teorias de campos, mais claramente no limite planar, com teorias de cordas, mas não ainda não é possível explicitar uma realização concreta desta relação. Foi preciso a introdução do conceito de branas para mudar este cenário. 


\subsection{A conjectura de Maldacena}

O trabalho de 't Hooft, revisitado acima, foi uma das primeiras motivações desta conjectura. Porém, até então, não era totalmente claro como tal dualidade poderia ser concretizada. A conjectura de Maldacena é de fato uma realização concreta desta dualidade "gravidade/calibre", ou seja, entre uma teoria de gravidade, ou cordas, e uma teoria quântica de campos. O ponto importante, que possibilitou esta realização, foi a introdução do conceito de branas. Uma vez motivada pelas descrições das branas a conjectura de Maldacena pôde ser construída, assim realizando concretamente esta dualidade. Faremos nesta seção uma breve revisão do conceito de branas, visando entender seus diferentes aspectos, como um hiper-plano no qual as cordas abertas devem ser presas, como também objetos que produzem efeitos gravitacionais gerando geometrias não triviais, sendo ambas as descrições relacionadas através afirmação da conjectura.

\subsubsection{D-branas e a conjectura}

As D-branas, objetos com mais dimensões que as cordas, passaram a ter um papel fundamental no estudo da teoria de cordas [18]. Estas também possuem um papel de extrema importância na correspondência AdS/CFT, sendo que a chave de sua importância reside no fato de que estas possuem duas possíveis descrições. A seguir, serão dadas motivações para a conjectura através desta dupla descrição das branas [19].

Uma D p-brana pode ser entendida como um objeto físico da teoria de cordas, assim como as próprias cordas fundamentais, porém, com sua extensão espacial vivendo em $p$ dimensões espaciais $x^{\mu}=\left(x^{0}, \ldots, x^{p}\right)$, sendo que denotaremos por $y^{i}=\left(x^{p+1}, \ldots, x^{9}\right)$ as direções transversais a estas. Tais como as cordas, as $\mathrm{D} p$-branas varrem uma região do espaço-tempo que chamaremos de volume-mundo, de $(p+1)$ dimensões. Além disso as Dbranas interagem com as cordas, emitindo ou absorvendo cordas fechadas que deslocam-se através delas e servindo como superfícies na quais cordas abertas devem ser presas. Uma corda aberta presa em uma Dp-brana é confinada à condição de que suas extremidades podem somente deslocar-se livremente nas $p$ dimensões em que a brana vive, não podendo mover-se nas direções transversais a esta hiper-superfície. 
Outra questão pertinente às branas são as condições de estabilidade destas, sendo que isto depende do contexto de qual teoria de cordas estas são inseridas. Para o caso de teorias do tipo IIA, por exemplo, somente branas com $p=0,2,4,6,8$ são estáveis, enquanto que para teorias do tipo IIB teremos somente $p=1,3,5,7 .^{2}$ O fato destas branas serem estáveis nestas teorias está relacionado à conservação das cargas de Ramond-Ramond (RR) das respectivas formas diferenciais acopladas. Uma vez que estas branas são os objetos carregados mais leves de cada teoria, a conservação de tais cargas não permite o decaimento destas.

A existência de tais objetos nos permite acessar o setor de cordas abertas, cujas extremidades estão presas a estas, que nos levam às teorias de calibre. No caso de cordas fechadas, temos que a sua quantização, no contexto de um espaço-tempo plano fixo, gera um espectro que leva a flutuações do espaço-tempo. Da mesma forma, é esperado que o espectro da quantização de cordas abertas, presas às branas, serão flutuações da própria D-brana em si. Outra forma mais geral de abordar a dinâmica das branas é através de uma ação efetiva, a ação de Born-Infield,

$$
S_{B I}=-T_{D p} \int d^{p+1} \sigma \sqrt{-\operatorname{det}\left(G_{\alpha \beta}+2 \pi \alpha^{\prime} F_{\alpha \beta}\right)}
$$

onde $T_{D p}$ é a tensão (ou densidade de energia) da brana. A tensão da brana é relacionada com a constante de acoplamento da corda fechada, na forma

$$
T_{D p}=\frac{1}{(2 \pi)^{2} g_{s} \ell_{s}^{p+1}} .
$$

Dada uma única Dp-brana, o respectivo espectro não-massivo, de mais baixa energia, de uma corda aberta presa a esta será:

- Um campos de calibre Abeliano $A_{\mu}(x)$, onde $\mu$ toma valores nas direções paralelas à brana, $\mu=0, \ldots, p$;

- $9-p$ campos escalares, $\Phi^{i}(x), i=1, \ldots, 9-p$;

- Campos fermiônicos, superparceiros do conteúdo bosônico.

\footnotetext{
${ }^{2}$ D9-branas também são possíveis, mas condições adicionais de consistência são necessárias.
} 
Uma vez que estes campos estão confinados nas dimensões em que a brana vive, é esperado que estes possuam dependência das coordenadas $x^{\mu}$. Uma possível interpretação física para os campos escalares $\Phi^{i}$ é de que estes representam as flutuações das branas nas direções transversas. De fato, além destes modos não massivos, existem outros modos massivos, com massas da ordem de $1 / \ell_{s}$, o inverso do comprimento típico de uma corda microscópica. Portanto, uma vez que os níveis de interesse sejam muito menores do que as massas do espectro massivo, nós podemos integrá-los fora e trabalhar somente com espectro nãomassivo.

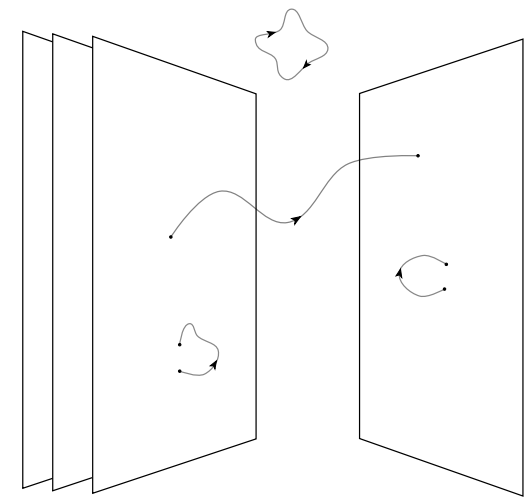

Figura 3.9: Construção de teorias não abelianas através de um conjunto de branas.

Para incorporar teorias de calibre não-Abelianas, é necessário trabalhar com mais do que uma única brana. Se considerado um conjunto sobreposto de $N$ branas, as diferentes combinações de como prender as extremidades das cordas será representada pelas cargas de Chan-Paton. Sendo o conjunto de branas enumerado, podemos indicar a quais branas as extremidades de uma determinada corda está presa através de dois índices, um para cada extremidade (um índice denotando uma representação fundamental para uma ponta e um índice anti-fundamental na outra), tomando valores de 1 a $N$. Uma ilustração disto pode ser vista na Figura 3.9. Estes graus de liberdade geram uma degenerescência de $N^{2}$, permitindo a construção de uma representação para $S U(N) \cdot{ }^{3}$ A configuração de interesse

\footnotetext{
${ }^{3}$ De fato o que se obtém é uma representação para $U(N)$, porém o fator $U(1)$ esta desacoplado do resto do grupo. A razão física para isto é que $U(1)$ descreve o movimento do centro de massa do conjunto de
} 
da conjectura é o caso em que consideramos uma única pilha de $N$ D3-branas no contexto da teoria de cordas tipo IIB. Neste caso, teremos que o conteúdo será:

- Um campo de calibre não-Abeliano, $\left(A_{\mu}\right)_{b}^{a}, \mu=0, \ldots, 3$;

- 6 campos escalares, $\left(\Phi^{i}\right)_{b}^{a}, i=1, \ldots, 6$;

- Superparceiros fermiônicos.

$a, b=1, \ldots, N$ são índices de $S U(N)$.

Este espectro é de fato o mesmo da $\mathcal{N}=4$ super Yang-Mills com o grupo de calibre $S U(N)$ em quatro dimensões, que será vista com mais detalhes na Seção (3.3). Uma outra forma de obter esta mesma ação é fazendo a expansão de campo fraco, potências de $F_{\alpha \beta}$, da ação de Born-Infield (3.15). Fazendo isto e identificando as constantes de acoplamento, segue que $g_{s}=g_{Y M}^{2}$. Devemos lembrar que nesta abordagem não estamos levando em consideração efeitos gravitacionais das branas, isto é possível olhando para o limite de $g_{s} N \ll 1$ (que, como veremos mais a frente, é equivalente à $L \ll \ell_{s}$ ), onde não teremos efeitos de reação da geometria de fundo, ou seja, teremos um espaço tempo plano.

Além disto, devemos levar em conta o setor que vem do espectro das cordas fechadas, que incluem interações inclusive com o as cordas abertas. Assim, podemos descrever a ação total deste sistema, de cordas e branas, como sendo:

$$
S=S_{\text {branas }}+S_{\text {interior }}+S_{\text {interações }}
$$

Porém, tomando o limite de $\ell_{s}=\sqrt{\alpha^{\prime}} \rightarrow 0$, conhecido como limite de Maldacena ou de baixas energias, nós teremos que os termos de interação, proporcionais à $G_{N} \propto \alpha^{\prime 2}$, poderão ser desprezados. Além disso, pelo mesmo motivo, a $S_{\text {interior }}$ é simplificada, restando apenas a gravidade livre num espaço plano $\mathbb{R}^{9,1}$. Por outro lado, a ação das branas, que dá origem à ação de super Yang-Mills, não é afetada por este limite. Isto se deve ao fato de que a constante de acoplamento da teoria, $g_{Y M}=\sqrt{g_{s}}$, não é afetada por este limite. Desta análise, tomando tais limites, a descrição das D3-branas resulta em:

$$
\mathcal{N}=4 \text { super Yang-Mills em } 4 \mathrm{~d} \text { com grupo } S U(N) \oplus \text { gravidade livre em } \mathbb{R}^{9,1} .
$$


Outra abordagem para o conjunto de D3-branas é possível se olharmos o limite inverso ao anterior, para $g_{s} N \gg 1\left(\right.$ ou $\left.L \gg \ell_{s}\right)$. Neste caso, o conjunto de D3-branas irá causar uma deformação da geometria do espaço-tempo. Portanto, os efeitos gravitacionais das branas não podem ser desprezados, e podemos interpretar estas branas em termos de soluções da equações de movimento da teoria de supergravidade do tipo IIB, dada pela ação

$$
S_{I I B}=\frac{1}{16 \pi G_{N}} \int d^{10} \sqrt{-g}\left(e^{-2 \phi}\left(R+4 \partial_{\mu} \phi \partial^{\mu} \phi\right)-\frac{1}{5 !} F_{5}^{2}\right),
$$

sendo que nesta equação estamos denotando por $\phi$ o dílaton e $F_{5}$ é o field strength da 4forma autodual. Para solucionar as equações do movimento desta ação, podemos procurar uma solução que preserve as mesmas simetrias preservadas pela brana, $S O(3,1) \times S O(6)$, sendo que o primeiro grupo diz respeito a simetrias de rotação e boosts sobre a brana e o segundo a simetrias de rotação nas direções transversais. A procura de tais soluções nos leva a uma solução da forma

$$
\begin{aligned}
d s^{2} & =H^{-1 / 2} \sum_{\mu, \nu=0}^{3} \eta_{\mu \nu} d x^{\mu} d x^{\nu}+H^{1 / 2}\left(d r^{2}+r^{2} d \Omega_{5}^{2}\right) \\
F_{5} & =(1+\star) d t \wedge d x^{1} \wedge d x^{2} \wedge d x^{3} \wedge d H^{-1} \\
\phi & =\phi_{0}=\text { constante }
\end{aligned}
$$

sendo que $\sum_{i}\left(d y^{i}\right)^{2}=d r^{2}+r^{2} d \Omega_{5}^{2}$ e $H$ é uma função só das coordenadas transversas. Supondo uma solução com simetria esférica chega-se a

$$
H(r)=1+\frac{L^{4}}{r^{4}}
$$

onde

$$
L^{4}=4 \pi g_{s} N \ell_{s}^{4}
$$

é a "carga" do conjunto de branas com relação à 4-forma. Para termos uma intuição física desta solução é interessante analisarmos alguns limites. Para $r \gg L$, a métrica volta a ser a métrica de Minkowski em dez dimensões, com pequenas correções da ordem

$$
\frac{L^{4}}{r^{4}} \sim \frac{N g_{s} \ell_{s}^{4}}{r^{4}} \sim \frac{G M}{r^{4}}
$$

sendo que usamos o fato de que $G \propto g_{s}^{2} \ell_{s}^{8}$ e interpretamos $N T_{D 3} \propto N / g_{s} \ell_{s}^{4}$ como a massa $M$ do conjunto de branas. Portanto, do ponto de vista das direções transversas, esta solução 
é um campo gravitacional gerado por um corpo de massa $N T_{D 3}$. Por outro lado, a região em que $r \ll L$ não possui uma geometria trivial, e esta região é chamada de garganta. Tomando o limite de $r \rightarrow 0$, também conhecido como limite próximo ao horizonte, a métrica se reduz a

$$
d s^{2}=\frac{r^{2}}{L^{2}}\left(-d t^{2}+d x_{1}^{2}+d x_{2}^{2}+d x_{3}^{2}\right)+\frac{L^{2}}{r^{2}} d r^{2}+L^{2} d \Omega_{5}^{2},
$$

onde podemos ver que a métrica se fatoriza em

$$
d s^{2}=d s_{A d S_{5}}^{2}+L^{2} d \Omega_{5}^{2}
$$

A partir da métrica acima, fazendo a mudança de coordenada $u=L^{2} / r$, é possível obter a métrica da forma (3.38). Além disso, tomando-se o limite de baixa energia, $\ell_{s}=\sqrt{\alpha^{\prime}} \rightarrow 0$ com $g_{s}$ e $N$ fixos, a dinâmica das cordas dentro da garganta desacopla das cordas no espaço plano. De forma intuitiva, neste limite, as cordas no espaço plano passam a ter um comprimento de onda muito grande, não conseguindo "enxergar" a região da garganta, enquanto que as cordas dentro da região da garganta não possuem energia suficiente para sair dela, ver Figura 3.10.

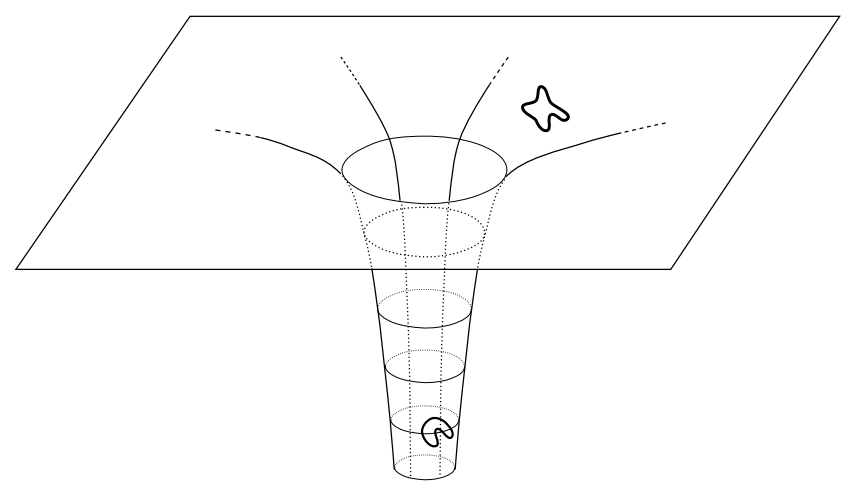

Figura 3.10: Espectro das cordas separadas pela 'garganta' de AdS.

Portante, ao analisar as D3-branas neste limites, o conteúdo obtido pode ser descrito 
como

Teoria de cordas tipo IIB na $A d S_{5} \times S^{5} \oplus$ gravidade livre em $\mathbb{R}^{9,1}$.

Uma vez considerado que as duas abordagens retratam o mesmo sistema, em limites diferentes, esta análise dá motivação para a conjectura de Maldacena, que estabelece a dualidade das duas teorias: A $\mathcal{N}=4$ super Yang-Mills com o grupo de calibre $S U(N)$ em quatro dimensões é dual à teoria de cordas tipo IIB na $A d S_{5} \times S^{5}$, sendo os parâmetros identificados como sendo

$$
g_{Y M}^{2}=g_{s}, \quad L^{4}=4 \pi g_{s} N \ell_{s}^{4}
$$

e o fluxo da 4-forma autodual através de uma esfera 5-dimensional identificado com o rank $N$ do grupo de calibre.

\section{$3.3 \mathcal{N}=4$ super Yang-Mills}

Como foi mencionado na seção anterior, a ação da $\mathcal{N}=4$ super Yang-Mills em 4 dimensões surge naturalmente como teoria efetiva do limite de baixas energias do volume-mundo de D3-branas. Esta teoria representa um dos lados da dualidade e portanto terá muita importância nesta dissertação. Dado sua importância, vamos fazer uma revisão dos aspectos gerais dela, sendo que uma revisão mais detalhada por ser encontrada em [20].

Esta teoria pode ser obtida a partir de uma redução dimensional da teoria $\mathcal{N}=1$ super Yang-Mills em dez dimensões, reduzindo 6 delas em $T^{6}$, ou seja, podemos obter a Lagrangiana da teoria a partir de ação

$$
S_{10}=\frac{1}{2 g_{Y M}^{2}} \int d^{10} x\left\{\operatorname{Tr}\left[F_{M N} F^{M N}-2 i \bar{\Psi} \Gamma^{M} D_{M} \Psi\right]\right\},
$$

denotando por $F_{M N}=\partial_{M} A_{N}-\partial_{N} A_{M}-i\left[A_{M}, A_{N}\right]$, onde os índices $M$ e $N$ assumem valores de 0 à $9, g_{Y M}$ é a constante de acoplamento de Yang-Mills, $A_{M}$ são os campos de calibre e $\Psi$ são espinores de Weyl-Majorana em dez dimensões (onde os índices espinoriais $\alpha$ e $\dot{\alpha}$ foram suprimidos). Como todos os campos fazem parte do mesmo supermultipleto, nós temos que todos os campos se transformam na representação adjunta de $S U(N)$. As 
transformações supersimétricas destes campos são:

$$
\begin{aligned}
\delta_{\epsilon} A_{M} & =\bar{\Psi} \Gamma_{M} \epsilon \\
\delta_{\epsilon} \Psi & =\frac{1}{2} F_{M N} \Gamma^{M N} \epsilon .
\end{aligned}
$$

Para obtermos a teoria em quatro dimensões, podemos fazer mais uma distinção útil entre os valores dos índices, sendo os índices gregos $\mu, \nu, \cdots=0, \ldots, 3$ e os índices latinos $i, j, \cdots=4, \ldots, 9$ (coordenadas em $T^{6}$, que faremos a redução dimensional), e assim podemos decompor $A_{M}=\left(A_{\mu}, \Phi_{i} \equiv A_{i}\right)$. Por simplicidade, vamos considerar somente os campos de calibre desta teoria. Podemos decompor as componentes do campo de calibre na forma

$$
F_{M N} F^{M N}=F_{\mu \nu} F^{\mu \nu}+2 F_{\mu j} F^{\mu j}+F_{i j} F^{i j}
$$

Do segundo termo da equação acima nós temos

$$
F_{\mu j} F^{\mu j}=\left(\partial_{\mu} \Phi_{j}-i\left[A_{\mu}, \Phi_{j}\right]\right)\left(\partial^{\mu} \Phi^{j}-i\left[A^{\mu}, \Phi^{j}\right]\right)=D_{\mu} \Phi_{j} D^{\mu} \Phi^{j}
$$

onde assumimos que nenhum dos campos dependem das coordenadas em $T^{6}$, ou seja, $\partial_{i} A_{\mu}=\partial_{i} \Phi_{j}=0$. Também definimos a derivada covariante como sendo $D_{\mu} \equiv \partial_{\mu}-i\left[A_{\mu},\right]$. Do terceiro termo da eq. (3.30) podemos obter que

$$
F_{i j} F^{i j}=\left(\partial_{i} \Phi_{j}-\partial_{j} \Phi_{i}-i\left[\Phi_{i}, \Phi_{j}\right]\right)\left(\partial^{i} \Phi^{j}-\partial^{j} \Phi^{i}-i\left[\Phi^{i}, \Phi^{j}\right]\right)=-\left[\Phi^{i}, \Phi^{j}\right]^{2}
$$

Juntando estes resultados com os campos fermiônicos da ação, e fazendo a integração nas coordenadas em $T^{6}$ (tal integração irá gerar um fator multiplicativo, que pode ser absorvido na definição da ação) e procedendo de maneira rigorosa é possível obter a ação da $S U(N) \mathcal{N}=4$ super Yang-Mills em quatro dimensões

$$
\begin{aligned}
S=\frac{1}{g_{Y M}^{2}} \int & d^{4} x \operatorname{Tr}\left(\frac{1}{2} F_{\mu \nu} F^{\mu \nu}+\frac{g_{Y M}^{2} \vartheta}{8 \pi^{2}} F_{\mu \nu} \tilde{F}^{\mu \nu}+D_{\mu} \Phi^{i} D^{\mu} \Phi^{i}\right. \\
& \left.+i \bar{\Psi} \Gamma^{\mu} D_{\mu} \Psi-\frac{1}{2}\left[\Phi^{i}, \Phi^{j}\right]\left[\Phi^{i}, \Phi^{j}\right]+i \bar{\Psi} \Gamma^{i}\left[\Phi^{i}, \Psi\right]\right)
\end{aligned}
$$

onde o ângulo $\vartheta$ está relacionado com termos topológicos. De fato, este termo contendo o ângulo $\vartheta$ não pode ser obtido a partir do processo de redução dimensional de uma teoria em dez dimensões, como descrito acima, e portanto é necessário acrescentá-lo ad hoc. Este termo é, por si só, supersimétrico mantendo a supersimetria da ação. 
Vamos analisar as várias simetrias presentes nesta teoria. Por construção, pela simetria $\mathcal{N}=4$, existe uma simetria-R $S U(4) \simeq S O(6)$ global, pela qual os campos de calibre formam um singleto, enquanto que os férmions e os escalares se transformam, respectivamente, nas representações $\mathbf{4}$ e $\mathbf{6}$.

Classicamente, esta ação é invariante sob transformações de escala, uma vez que todos os termos na Lagrangiana possuem dimensão 4. De fato, mesmo a nível quântico ela também é conformemente invariante, uma vez que a função beta da teoria é igual a zero. O grupo conforme, isomórfico à $S O(4,2) \simeq S O(6)$, é composto pelas transformações de Poincaré, dilatações e especiais conformes. Mais detalhes deste grupo se encontram no Apêndice IV. Este grupo combinado com as 16 supercargas de Poincaré, devido à $\mathcal{N}=4$, forma o grupo superconforme $S U(2,2 \mid 4)$. O grupo também possui, em adição às 16 supercargas de Poincaré $Q_{\alpha}^{a}$ e $\bar{Q}_{a \dot{\alpha}}, 16$ cargas superconformes $S_{\alpha}^{a}$ e $\bar{S}_{a \dot{\alpha}}{ }^{4}$ devido ao fato de que a supersimetria de Ponicaré e as transformações especiais conformes não comutarem.

Mais do que isso, sabemos que a invariância superconforme existe mesmo a nível quântico e que a teoria é UV finita, de fato a função $\beta$ da teoria é igual à zero em todas as ordens como pode ser visto nas referências [21]. Como consequência, a constante de acoplamento $g_{Y M}$ é, na realidade, um parâmetro que pode ser escolhido e fixado. Assim a $\mathrm{SYM} \mathcal{N}=4$ é unicamente definida apenas através do valor de $g_{Y M}$ e o rank do grupo de calibre $N$.

\subsection{Espaço AdS}

Agora, vamos revisar alguns aspectos do segundo lado da correspondência, o espaço Anti de Sitter. Também conhecido como espaço AdS, esta é a geometria do espaço tempo que, como foi visto na Seção 3.2.1, foi encontrada como solução da métrica devido a presença das D3-branas. De fato, a solução da métrica encontrada possui uma parte da métrica referente à esfera $S^{5}$, que, de forma geral, não irá ser de grande relevância em nossas cálculos e portanto não vamos dar-lhe muitos detalhes.

\footnotetext{
${ }^{4}$ Aqui os índices $a$ índices da simetria-R, enquanto que $\alpha$ e $\dot{\alpha}$ são índices de spinores de Weyl.
} 
Um jeito que podemos imaginar o espaço AdS é por meio de coordenadas imersas. Da mesma forma que podemos descrever a superfície de uma esfera, de raio $L$, em termos de coordenadas em três dimensões, $\left(X_{1}, X_{2}, X_{3}\right)$, na forma

$$
X_{1}^{2}+X_{2}^{2}+X_{3}^{2}=L^{2},
$$

podemos também imaginar o espaço $A d S_{5}$ como sendo um lugar geométrico tal que suas coordenadas imersas $X_{M}(\operatorname{com} M=-1,0, \ldots, 4)$, obedecem a restrição

$$
-X_{-1}^{2}-X_{0}^{2}+\sum_{k=1}^{4} X_{k}^{2}=-L^{2},
$$

que definem um hiperboloide em $\mathbb{R}^{4,2}$, sendo que este espaço ambiente possui duas "coordenadas temporais", e $L$ é o raio do espaço. Importante notarmos que, apesar de usarmos duas coordenadas temporais para as coordenadas imersas, a superfície AdS somente irá conter uma única coordenada temporal.

Desta definição, podemos ver explicitamente que o grupo de isometria deste espaço deve ser $S O(4,2)$. Lembrando que ao juntarmos com a métrica referente à $S^{5}$, que também faz parte da solução encontrada, nós teremos o grupo de isometria total de $S O(4,2) \times S O(6)$. Para obtermos a métrica induzida na hipersupefície, vamos reescrever as coordenadas $X_{i}$

$$
X_{-1}+X_{4}=\frac{L}{z}, \quad X_{\mu}=\frac{L}{z} x_{\mu}, \quad \mu=0, \ldots, 3,
$$

sendo que aqui introduzimos a chamada coordenada radial de $A d S_{5}, z \in[0, \infty)$. Calculando a métrica induzida na superfície AdS através da fórmula geral

$$
g_{\alpha \beta}=G_{M N} \partial_{\alpha} X^{M} \partial_{\beta} X^{N},
$$

onde os índices $\alpha$, $\beta$ são índices que denotam as coordenadas da superfície, em nosso caso $\mu, \nu$ e $z$, enquanto que $M, N$ são índices das coordenadas imersas. Podemos calcular a métrica induzida usando as relações (3.35) e (3.36), fazendo isso nós temos

$$
d s^{2}=g_{\alpha \beta} d x^{\alpha} d x^{\beta}=\frac{L^{2}}{z^{2}}\left(d z^{2}+\eta_{\mu \nu} d x^{\mu} d x^{\nu}\right),
$$

que é conhecida como a métrica no Poincaré patch. Observamos que na hipersuperfície voltamos a ter apenas uma coordenada temporal. Nesta métrica, nós chamamos de borda de $A d S_{5}$ a região em que $z=0$. 
Podemos utilizar outro sistema de coordenadas para descrever $A d S_{5}$, definindo

$$
X_{-1}=L \cosh \rho \cos t, \quad X_{0}=L \cosh \rho \sin t, \quad X_{k}=L \sinh \rho \Omega_{k},
$$

$\operatorname{com} \sum_{k=1}^{4} \Omega_{k}^{2}=1$. Com estas coordenadas, obtemos o que chamamos de métrica em coordenadas globais

$$
d s^{2}=L^{2}\left(-\cosh ^{2} \rho d t^{2}+d \rho^{2}+\sinh ^{2} \rho d \Omega_{k}\right)
$$

sendo que para $t \in[0,2 \pi)$ e $\rho \in(0, \infty)$ nós podemos cobrir todo o hiperboloide, Figura 3.11. Porém, escolhendo este domínio para a variável temporal e tomando $\rho \rightarrow 0$

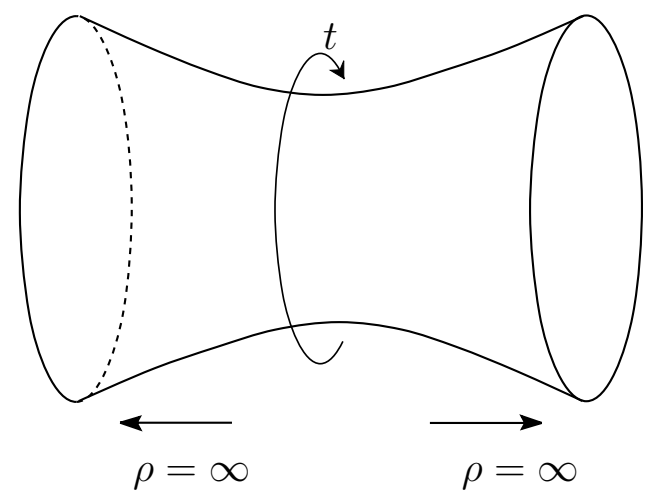

Figura 3.11: Espaço AdS como um hiperboloide nas coordenadas globais $\rho, t$ e $\Omega_{k}$.

somos levados a uma coordenada temporal cíclica, que nos gera problemas de curvas fechadas do tipo tempo [22]. ${ }^{5}$ Para evitarmos este problema, podemos definir de forma natural um domínio para $t$ tal que $t \in(-\infty,+\infty)$, chamado de cobertura universal.

Outra forma útil de escrevermos esta métrica é fazendo a mudança de coordenadas

$$
\tan \theta=\sinh \rho, \quad \theta \in\left[0, \frac{\pi}{2}\right)
$$

Assim podemos escrever a métrica na forma

$$
d s^{2}=-d t^{2}+d \theta^{2}+\sin \theta^{2} d \Omega_{k}^{2}
$$

\footnotetext{
${ }^{5}$ Uma discussão na correspondência AdS/CFT está em [23].
} 


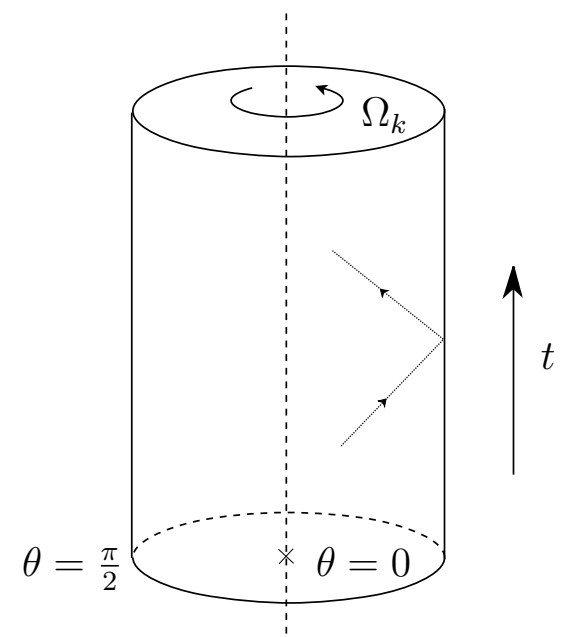

Figura 3.12: Espaço AdS como um cilindro em coordenadas $\theta, t$ e $\Omega_{k}$, e uma representação de uma trajetória do tipo luz atingindo a borda de AdS.

Escrita desta forma, podemos visualizar o espaço AdS como um cilindro, cuja borda se encontra em $\theta=\pi / 2$, ver Figura 3.12. Daqui, podemos ver diretamente que para trajetórias do tipo tempo nós teremos

$$
d s^{2}=0 \quad \Rightarrow \quad \int_{0}^{T} d t=\int_{0}^{\pi / 2} d \theta=\frac{\pi}{2} .
$$

Portanto, qualquer intervalo de tempo $T$ de uma trajetória do tipo tempo é finito, pois neste caso o lado direito da equação acima está entre $[0, \pi)$. Disto, podemos ver que partículas cuja trajetória seja do tipo luz, como o gráviton, podem atingir a borda do espaço em tempo finito. Desta análise, podemos concluir que precisaremos da informação das condições de contorno na borda do espaço, para assim definir completamente os campos neste espaço.

Esta necessidade de definição das condições de contorno na borda de AdS é um dos pontos chaves da correspondência. Como discutido na Seção 3.1.1, a teoria de campos, que será definida na borda do espaço, irá nos dar tais condições de contorno, como veremos com mais detalhes na Seção 3.6. 


\subsection{Verificações das simetrias globais}

Como vimos, a $\mathcal{N}=4$ super Yang-Mills em 4 dimensões possui a simetria bosônica conforme $S O(4,2) \simeq S U(2,2)$, e juntamente com a simetria-R $S U(4) \simeq S O(6)$, devido à $\mathcal{N}=4$, forma o grupo de simetria superconforme $S U(2,2 \mid 4)$, englobando o subgrupo $S O(4,2) \times S O(6)$. Como observamos na subseção anterior, este subgrupo é exatamente o grupo de simetria do espaço $A d S_{5} \times S^{5}$. Temos que $S O(4,2)$ atua como uma simetria conforme em quatro dimensões na borda do espaço $A d S_{5}$, onde é visto como o próprio grupo de isometria, enquanto que a simetria $S O(6)$, a principio relacionada à simetria$\mathrm{R}$ da supersimetria, agora é identificada com o grupo de isometria da $S^{5}$. Além disso, utilizando as equações de espinores de Killing, pode-se verificar que a métrica $A d S_{5} \times S^{5}$ é maximalmente supersimétrica, conservando todas as 32 supersimetrias da teoria de cordas do tipo IIB. Estas supersimetrias conservadas por esta métrica, por sua vez, pode ser identificada com as 32 supercargas da teoria de calibre.

Além destas simetrias globais, ainda temos o fato de que ambas as teorias possuem a simetria $S L(2, \mathbb{Z})$. No caso da teoria de calibre, isto é realizado na transformação da constante de acoplamento complexa $\tau \equiv \vartheta / 2 \pi+4 \pi i / g_{Y M}^{2}$, como uma transformação modular (também conhecida como dualidade-S). Por outro lado, esta transformação é realizada na teoria de cordas agindo no campo do axion-dílaton $\tau \equiv C_{0}+i e^{-\phi}$.

A checagem das simetrias globais é essencial para sustentabilidade mínima da correspondência. Isto se deve ao fato de que simetrias globais estão relacionadas a correntes e cargas conservadas. Estas possuem uma natureza física, sendo assim necessário que, se de fato existe uma correspondência, se conservem em ambas as teorias. Por outro lado, simetrias locais, como a simetrias de calibre e difeomorfismos, não estão relacionadas a quantidades físicas reais, em geral são somente formas diferentes de abordar os mesmo sistemas físicos.

\subsection{Dicionário}

Nas subseções anteriores, nós demos argumentos que suportam a existência da conjectura de Maldacena, além de uma revisão das duas teorias em questão, faltando agora estabele- 
cer explicitamente a forma como vamos relacionar os objetos de cada teoria. Essa relação, entre as entidades físicas de cada teoria é denominada de dicionário da correspondência. Nesta subseção vamos apresentar de forma sucinta alguns dos elementos deste dicionário, onde daremos definições e relações que são de uso constante em cálculos de objetos utilizando a correspondência.

Uma vez que estamos trabalhando com uma teoria de campos conforme, não faz sentido falarmos de estados assintóticos, já que, por conta da simetria conforme, não existe sentido em falar de estados separados por grandes distâncias. Portanto, não podemos pensar em teorias de espalhamento usuais. Neste caso, os observáveis da teoria são as funções de correlação dos operadores, em geral locais, no vácuo, objetos da forma

$$
\left\langle\mathcal{O}_{1}\left(x_{1}\right) \mathcal{O}_{2}\left(x_{2}\right) \ldots \mathcal{O}_{n}\left(x_{n}\right)\right\rangle
$$

Para calcular tais quantidades vamos utilizar uma função geratriz $Z[J]$, como fazemos usualmente, sendo que esta irá conter a Lagrangiana original somada a uma perturbação, cuja fonte podemos denotar genericamente por $J(x)$,

$$
\mathcal{L}(x)+\sum_{n} J_{n}(x) \mathcal{O}(x) \equiv \mathcal{L}+\mathcal{L}_{J}
$$

o que nos dá uma função geratriz da forma

$$
Z[J]=\left\langle e^{-\int \mathcal{L}_{J} d x}\right\rangle_{\mathrm{CFT}}
$$

Desta, obtemos as funções de correlação, como de costume, fazendo as derivadas funcionais:

$$
\left\langle\prod_{n} \mathcal{O}_{n}\left(x_{n}\right)\right\rangle=\left.\prod_{n} \frac{\delta}{\delta J_{n}\left(x_{n}\right)} \ln Z\right|_{J=0} .
$$

Porém, para fazer a ligação entre os campos e os operadores, nós podemos interpretar as fontes $J$ como sendo os campos no interior da borda, ou seja, ao invés da expressão genérica (3.45), nós podemos escrever

$$
\mathcal{L}+\mathcal{L}_{J} \rightarrow \mathcal{L}+\sum_{n} \phi_{n}(x) \mathcal{O}_{n}(x)
$$

onde estamos denotando por $\phi_{n}(x)$ qualquer campo do interior da teoria avaliado na borda da AdS, ou seja,

$$
\phi(x)=\left.\Phi(x)\right|_{\partial A d S} \sim \lim _{z \rightarrow 0} \Phi(x, z),
$$


onde a forma específica do lado direito da equação depende do tipo de operador associado ao campo. Desta forma, podemos fazer uma associação de cada campo no interior da AdS com um operador invariante de calibre local, sendo esta relação chamada de correspondência operador/campo. Entretanto, esta associação entre operadores e campos não possui um regra geral bem definida, sendo que o que se faz usualmente é impor que ambos tenham os mesmo números quânticos com respeito às simetrias globais. Um exemplo muito importante desta associação é o caso da métrica $g_{\mu \nu}(x)$ que está associado com o operador densidade de energia-momento $T^{\mu \nu}$

$$
\int d^{4} x g_{\mu \nu}(x) T^{\mu \nu}(x)
$$

Neste caso, vemos que o tensor energia-momento, relacionado com a corrente devido à invariância translacional da teoria conforme, está relacionado com os valores da métrica $g_{\mu \nu}(x, z)$ na borda da teoria de gravidade. Disto, temos que de forma geral teorias que possuem invariância translacional estão relacionadas com teorias cuja gravidade é dinâmica e vice-versa.

De fato, este tipo de associação entre campos de calibre da teoria de gravidade e correntes conservadas na teoria conforme é assumida muitas vezes, de forma geral, escrita como

$$
\int d^{4} x A_{\mu}(x) J^{\mu}(x)
$$

onde $A_{\mu}(x)$ é algum campo de calibre da teoria de gravidade avaliado na borda e $J^{\mu}(x)$ é alguma corrente conservada na teoria conforme. Este tipo de relação muitas vezes é dita como sendo uma correspondência entre as simetrias globais da teoria que vivem na borda com simetrias de calibre da teoria do interior da respectiva borda.

Tal identificação das fontes das perturbações com os campos da teoria de gravidade é importante uma vez que na correspondência nós podemos identificar as funções geratrizes das duas teorias, mais explicitamente expressa na fórmula de GKPW [2,3]

$$
Z_{\mathrm{CFT}}[\phi(x)]=Z_{\text {cordas }}\left[\left.\Phi(x)\right|_{\partial A d S}\right]
$$

A princípio, a ação envolvida no lado direito da equação é muito complicada, mas podemos simplifica-lá se tomarmos o limite de $N \rightarrow \infty, \operatorname{com} \lambda=g_{Y M}^{2} N$ fixo, sendo levados à teoria 
clássica de cordas do tipo IIB, ao invés de quântica. Isso pode ser entendido intuitivamente a partir da análise feita em (3.1.3), onde vemos que neste limite $g_{s} \rightarrow 0$ e assim podemos desconsiderar efeitos de loops das cordas. Além disso, podemos ainda escolher o limite de $\lambda \rightarrow \infty$, o que é equivalente à $\alpha^{\prime} \rightarrow 0$ ou $\ell_{s}^{2} \sim m_{s} \rightarrow 0\left(m_{s}\right.$ é a massa dos estados massivos), fazendo com que possamos desprezar a torre de estados massivos proveniente da teoria de cordas do tipo IIB, e assim ficando somente com uma respectiva teoria clássica de supergravidade. Outra forma de interpretar este limite é lembrando que $T_{s} \sim 1 / \alpha^{\prime}$, ou seja, a tensão da corda vai a infinito, portanto podemos desprezar as oscilações da corda clássica e assim podemos utilizar a ação de Nambu-Goto clássica. Esta última forma de ver o limite de $\lambda$ grande será importante para o cálculo dos laços de Wilson holográficos, onde iremos utilizar cordas macroscópicas, descrevendo-as com a ação de Nambu-Goto com condições de contorno não triviais.

Tendo utilizado estes limites, e simplificado o lado direito da (3.52)

$$
Z_{\text {cordas }}\left[\left.\Phi(x)\right|_{\partial A d S}\right] \simeq Z_{\mathrm{SUGRA}}\left[\left.\Phi_{\text {class }}(x)\right|_{\partial A d S}\right]
$$

nós podemos escrever a fómula (3.47) em termos dos campos clássicos

$$
\left\langle\prod_{n} \mathcal{O}_{n}\left(x_{n}\right)\right\rangle=\left.\prod_{n} \frac{\delta S_{\mathrm{SUGRA}}\left[\left.\Phi_{\text {class }}(x)\right|_{\partial A d S}\right]}{\delta \phi_{n}\left(x_{n}\right)}\right|_{\phi=0} .
$$

Aqui finalizamos a apresentação de alguns dos elementos do dicionário da correspondência. 


\section{Capítulo 4}

\section{Laços de Wilson supersimétricos}

Neste capítulo faremos a extensão da definição de laços de Wilson para a teoria de calibre $\mathcal{N}=4$ superYang-Mills, assim como a sua computação em si. Como foi dito na Seção 3.1, estes objetos são de grande interesse para o estudo da correspondência. A importância destes se deve ao fato de que nos providenciam uma verificação não trivial da correspondência, sendo que ainda não temos uma demonstração formal da correspondência. Mas além disso, como veremos, estes objetos são por si próprios de interesse no contexto da $\mathcal{N}=4$ super Yang-Mills, uma vez que é possível serem calculados exatamente, não só em limites perturbativos. Além disso, neste capítulo também iremos encontrar as condições de supersimetria para estes operadores, achando restrições para o seu contorno. Prosseguindo os cálculos, teremos que estes irão se reduzir ao problema de se contar o número de diagramas planares que podemos construir, dado um número de propagadores fixo. Para resolver este problema, vamos utilizar técnicas que serão apresentadas no Capítulo 5.

\subsection{Definindo o laço de Wilson supersimétrico}

Como foi dito anteriormente no Capítulo 2, o laço de Wilson é caracterizado pelo contorno $C$, sobre o qual será feita a integração dos campos de calibre e a representação $R$ do grupo de calibre. Neste contexto de uma teoria supersimétrica, é natural definirmos o contorno $C$ sobre um espaço estendido com coordenadas que se acoplem aos campos do 
supermultipleto. Tais coordenadas serão $\left\{x^{\mu}(\tau), y^{i}(\tau), \xi(\tau)\right\}$, sendo $x^{\mu}$ e $y^{i}$ direções bosônicas que se acoplam com os campos $A^{\mu}$ e $\Phi^{i}$, enquanto que $\xi(\tau)$ são direções fermiônicas que se acoplam com os espinores $\Psi$. Uma forma de interpretar estas coordenadas extras é lembrando que, como vimos na Seção 3.3, a teoria pode ser obtida a partir de uma teoria em dez dimensões, assim as coordenadas $y^{i}$ podem ser interpretadas como as coordenadas da dimensão reduzida de $T^{6}$.

Devemos lembrar que, por causa da invariância de calibre, devemos ter um circuito fechado nas coordenadas $x^{\mu}$, enquanto que a princípio podemos ter valores arbitrários para $y^{i}$ e $\xi$. De fato, ainda podemos simplificar a forma do operador não incluindo as direções fermiônicas, pois, de acordo com [24], os operadores contendo $\Psi$ são descendentes supersimétricos do operador que não os contém. Com isto em mente, um ansatz pelo operador laço de Wilson supersimétrico pode ser tomado (na assinatura euclideana), ${ }^{1}$ como

$$
W_{R}(C)=\frac{1}{\operatorname{dim} R} \operatorname{Tr}_{R} \mathcal{P} \exp \oint_{C} d \tau\left[i A_{\mu}(\tau) \dot{x}^{\mu}+\Phi^{i}(\tau) \dot{y}^{i}\right],
$$

onde $\operatorname{Tr}_{R}$ significa que estamos deixando o traço livre para ser calculado na representação irredutível $R$ de $S U(N)$, que podemos interpretar como a representação da partícula de prova que se acopla com o campo de calibre. Neste trabalho vamos nos restringir ao cálculo do operador somente à representação fundamental, $R=\square \operatorname{com} \operatorname{dim} \square=N$, e portanto não vamos mais escrever esta etiqueta explicitamente.

\subsection{Condições de supersimetria}

Para que estes sejam de fato operadores supersimétricos, temos que analisar o comportamento destes sob as transformações supersimétricas de Poincaré e conformes. Podemos obter as transformações supersimétricas, primeira linha de (4.2), destes a partir das transformações dos campos de calibre e escalares que podem ser obtidas de (3.29)

$$
\delta_{Q} A_{\mu}=\bar{\Psi} \Gamma_{\mu} \epsilon_{0}, \quad \delta_{Q} \Phi^{i}=\bar{\Psi} \Gamma^{i} \epsilon_{0}
$$

\footnotetext{
${ }^{1} \mathrm{Na}$ assinatura Lorentziana teríamos o fator de $i$ multiplicando os campos escalares. A modificação para a definição no caso euclideano ficará mais clara quanto obtivermos a condição de supersimetria.
} 
Além destas transformções, também temos as transformações superconformes

$$
\delta_{S} A_{\mu}=\bar{\Psi} \Gamma_{\mu} x^{\nu} \Gamma_{\nu} \epsilon_{1}, \quad \delta_{S} \Phi^{i}=\bar{\Psi} \Gamma^{i} x^{\nu} \Gamma_{\nu} \epsilon_{1}
$$

Aqui os parâmetros $\epsilon_{0,1}$ são dois férmions de Majorana-Weyl de 16 componentes (assim como os $\Psi$ ) de quiralidades opostas. Primeiramente, observando os efeitos das transformações devidas as supercargas de Poincaré, temos que

$$
\begin{aligned}
\delta_{Q} W(C) & =\frac{1}{N} \operatorname{Tr} \mathcal{P}\left[\delta_{Q}\left(i A_{\mu}(\tau) \dot{x}^{\mu}+\Phi^{i}(\tau) \dot{y}^{i}\right) \exp \oint_{C} d \tau\left[i A_{\mu}(\tau) \dot{x}^{\mu}+\Phi^{i}(\tau) \dot{y}^{i}\right]\right] \\
& =\frac{1}{N} \operatorname{Tr} \mathcal{P}\left[\bar{\Psi}\left(i \Gamma_{\mu} \dot{x}^{\mu}+\Gamma^{i} \dot{y}^{i}\right) \epsilon_{0} \exp \oint_{C} d \tau\left[i A_{\mu}(\tau) \dot{x}^{\mu}+\Phi^{i}(\tau) \dot{y}^{i}\right]\right]
\end{aligned}
$$

ou seja, para que tenhamos $\delta_{Q} W(C)=0$, invariância sob tais transformações, nós temos que ter

$$
\left(i \Gamma^{\mu} \dot{x}_{\mu}+\Gamma^{i} \dot{y}^{i}\right) \epsilon_{0}=0
$$

Para $\tau$ fixo, nós teremos oito soluções caso o quadrado do operador $\left(i \Gamma^{\mu} \dot{x}_{\mu}+\Gamma^{i} \dot{y}^{i}\right)$ se anule. Impondo tal condição nós temos

$$
\begin{aligned}
\left(i \Gamma^{\mu} \dot{x}_{\mu}+\Gamma^{i} \dot{y}^{i}\right)\left(i \Gamma^{\nu} \dot{x}_{\nu}+\Gamma^{j} \dot{y}^{j}\right)= & -\frac{\left(\Gamma^{\mu} \Gamma^{\nu}+\Gamma^{\nu} \Gamma^{\mu}\right)}{2} \dot{x}^{\mu} \dot{x}^{\nu}+\left(i \Gamma^{\mu} \Gamma^{j}+i \Gamma^{j} \Gamma^{\mu}\right) \dot{x}_{\mu} \dot{y}^{j} \\
& +\frac{\left(\Gamma^{i} \Gamma^{j}+\Gamma^{j} \Gamma^{i}\right)}{2} \dot{y}^{i} \dot{y}^{j} \\
= & -\frac{\left\{\Gamma^{\mu}, \Gamma^{\nu}\right\}}{2} \dot{x}^{\mu} \dot{x}^{\nu}+\left\{\Gamma^{\mu}, \Gamma^{j}\right\} i \dot{x}_{\mu} \dot{y}^{j}+\frac{\left\{\Gamma^{i}, \Gamma^{j}\right\}}{2} \dot{y}^{i} \dot{y}^{j} .
\end{aligned}
$$

Utilizando as propriedades dos anticomutadores das matrizes $\Gamma,\left\{\Gamma^{M}, \Gamma^{N}\right\}=2 g^{M N}$ (lembrando que $M, N=\mu, \nu$ ou $i, j$ ) chegamos à condição ${ }^{2}$

$$
\left(i \Gamma^{\mu} \dot{x}_{\mu}+\Gamma^{i} \dot{y}^{i}\right)^{2}=0 \quad \Longleftrightarrow \quad \dot{y}^{2}-\dot{x}^{2}=0
$$

Isto pode ser solucionado fazendo $\dot{y}^{i}(\tau)=|\dot{x}| \theta^{i}(\tau)$, onde $\theta^{i}$ é um vetor na esfera unitária em cinco dimensões $\left(\theta^{2}=1\right)$. Podemos fazer a mesma análise sob a transformação superconforme e assim obter a mesma condição. Notemos que o operador $\left(i \Gamma^{\mu} \dot{x}_{\mu}+\Gamma^{i}|\dot{x}| \theta^{i}(\tau)\right)$

\footnotetext{
${ }^{2}$ No caso da assinatura Lorentziana teríamos a condição equivalente $\dot{y}^{2}+\dot{x}^{2}=0$, que possui sentido nessa assinatura e que permite existir laços de Wilson sem acoplamento aos escalares $\left(\dot{y}^{2}=0\right)$ e ao longo de uma direção de tipo luz $\left(\dot{x}^{2}=0\right)$.
} 
possui dependência de $\tau$, e isso implicaria numa supersimetria local, sendo que estamos supondo uma teoria que possui simetria global (os parâmetros $\epsilon_{0,1}$ não dependem de $\tau$ ). Portanto, nós temos que escolher uma direção fixa para $\theta^{i}$ e impor que $\dot{x}^{\mu}$ seja uma constante, ou seja, o contorno $C$ será uma linha reta, que deve ser infinitamente comprida para garantir invariância de calibre. Outro contorno possível é o contorno circular, que pode ser obtido através de uma transformação conforme, mais especificamente a transfornação de inversão, conservando o mesmo número de supercargas. Com tais restrições, nós teremos que $W(C)$ comutará com metade das super cargas, o que na literatura é conhecido como '1/2 BPS'. 3

Utilizando esta solução na (4.1), nós obtemos a seguinte expressão para o laço de Wilson supersimétrico $1 / 2$ BPS

$$
W(C)=\frac{1}{N} \operatorname{Tr} \mathcal{P} \exp \oint_{C} d \tau\left[i A_{\mu}(\tau) \dot{x}^{\mu}+\Phi^{i}(\tau)|\dot{x}| \theta^{i}\right],
$$

onde $C$ é uma linha reta infinita ou um círculo.

\subsection{Laços de Wilson em teoria perturbativa}

A computação dos laços de Wilson será realizada através de modelo de matrizes, como iremos explicar na próxima seção. Historicamente [25], a utilização do modelo de matrizes foi proposta e motivada pelas evidências sugeridas pela computação perturbativa que veremos nesta seção.

Primeiramente, para calcularmos tais operadores, vamos fazer uso da técnica padrão de cálculo perturbativo em teorias de campos. Podemos expandir o operador (4.8), utilizando a fórmula (2.11), em potências de $g_{Y M}$. Fazendo esta expansão e em seguida tomando o VEV de cada termo, podemos obter uma expansão da forma

$$
\left\langle W_{C}\right\rangle=\sum_{n=0}^{\infty} C_{n}
$$

\footnotetext{
${ }^{3}$ Aqui o nome 'BPS' se deve somente ao fato deste operador conservar algumas das supercargas, não estamos nos referindo à relação de saturação entre a massa e a carga central, como é normalmente utilizado.
} 
$\operatorname{com}^{4}$

$$
\begin{aligned}
C_{0} & =1, \\
C_{1} & =\int d \tau_{1} \int^{\tau_{1}} d \tau_{2} \frac{1}{N} \operatorname{Tr}\left[-\dot{x}_{1}^{\mu} \dot{x}_{2}^{\nu}\left\langle A_{\mu}\left(x_{1}\right) A_{\nu}\left(x_{2}\right)\right\rangle+\left|\dot{x}_{1}\right| \theta^{i}\left|\dot{x}_{2}\right| \theta^{j}\left\langle\Phi_{i}\left(x_{1}\right) \Phi_{j}\left(x_{2}\right)\right\rangle\right] \\
C_{2} & =\ldots,
\end{aligned}
$$

onde estamos denotamos $x_{n}=x\left(\tau_{n}\right)$ e o fator 2 ! em $C_{1}$, como acontecerá para os outros fatores de $n$ !, foi cancelado pelo fixamento da ordem das integrais, ver (2.11). O termo $C_{0}$ pode ser obtido observando que a potência de ordem zero do operador (4.8) nos dará a identidade. Tomando o traço da identidade na representação fundamental dá $\operatorname{Tr} 1=N$, o que cancela com o denominador $1 / N$, sendo o resultado $C_{0}=1$.

Vamos agora calcular $C_{1}$, a primeira contribuição não trivial, tomando cuidado para entender a origem de cada fator, uma vez que os termos de ordens mais altas serão simplesmente uma generalização deste cálculo.

Utilizando o valor dos propagadores dos campos, listados no Apêndice II, e lembrando que $A_{\mu}\left(x_{1}\right)=T^{a} A_{\mu}^{a}\left(x_{1}\right)$, sendo $T^{a}$ as matrizes geradoras na representação fundamental de $S U(N)$, sendo que aqui os índices $a, b \ldots$ referem-se aos geradores de $S U(N)$, que vai de 1 à $N^{2}-1$, nós temos que

$$
\begin{aligned}
C_{1} & =\frac{1}{N} \int d \tau_{1} \int^{\tau_{1}} d \tau_{2} \operatorname{Tr}\left(T^{a} T^{b}\right)\left\{-\dot{x}_{1}^{\mu} \dot{x}_{2}^{\nu} \frac{g_{Y M}^{2} \delta^{a b}}{4 \pi^{2}} \frac{\delta_{\mu \nu}}{\left|x_{1}-x_{2}\right|^{2}}+\left|\dot{x}_{1}\right| \theta^{i}\left|\dot{x}_{2}\right| \theta^{j} \frac{g_{Y M}^{2} \delta^{a b}}{4 \pi^{2}} \frac{\delta_{i j}}{\left|x_{1}-x_{2}\right|^{2}}\right\} \\
& =\frac{g_{Y M}^{2}}{\left(4 \pi^{2}\right) N} \int d \tau_{1} \int^{\tau_{1}} d \tau_{2} \operatorname{Tr}\left(T^{a} T^{a}\right) \frac{\left|\dot{x}_{1}\right|\left|\dot{x}_{2}\right|-\dot{x}_{1} \cdot \dot{x}_{2}}{\left|x_{1}-x_{2}\right|^{2}} .
\end{aligned}
$$

Para o cálculo do traço, podemos utilizar a identidade

$$
T^{a} T^{a}=\frac{N}{2}
$$

Portanto, ao efetuarmos o traço, nós vamos obter um fator de $N^{2}$, sendo que um deles irá se cancelar com o denominador. O que resulta em

$$
C_{1}=\frac{g_{Y M}^{2} N}{2\left(4 \pi^{2}\right)} \int d \tau_{1} \int^{\tau_{1}} d \tau_{2} \frac{\left|\dot{x}_{1}\right|\left|\dot{x}_{2}\right|-\dot{x}_{1} \cdot \dot{x}_{2}}{\left|x_{1}-x_{2}\right|^{2}} .
$$

Podemos representar este termo em forma de um diagrama. Uma vez que sempre teremos contribuições de forma dupla, proveniente do campo escalar e de calibre, desenharemos um

\footnotetext{
${ }^{4} \mathrm{O}$ termo linear nos campos não contribui pois $\operatorname{Tr} T^{a}=0$.
} 
único propagador que representará a contribuição dos dois campos, escalar e de calibre. Além disso, desenharemos um círculo que representará o contorno do laço de Wilson, como ilustrado na Figura 4.1. Esta representação será útil para visualizar o comportamento dos diagramas planares, que serão visto na Seção 4.5.1.

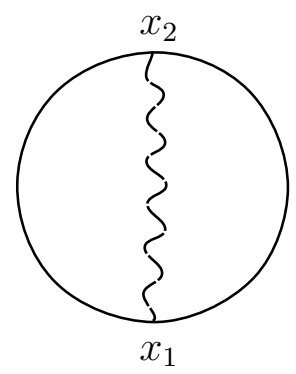

Figura 4.1: Representação diagramática de $C_{1}$, onde o propagador representa ambos os campos, escalar e de calibre, enquanto o círculo representa o contorno do laço.

\subsubsection{Divergência do propagador}

Aqui devemos ter certo cuidado, uma vez que os propagadores possuem uma divergência ultra-violeta em $x_{1}=x_{2}$, temos que verificar se a divergência reside em $C_{1}$. Veremos isto com mais detalhes. A contribuição do propagador do campo de calibre é dado por

$$
-\frac{g_{Y M}^{2} N}{8 \pi^{2}} \int d \tau_{1} \int d \tau_{2} \dot{x}_{1}^{\mu} \dot{x}_{2}^{\nu} \frac{\delta_{\mu \nu}}{\left|x_{1}-x_{2}\right|^{2}} .
$$

Estamos interessados na contribuição deste termo próximo do ponto em que $x_{1}=x_{2}$ (utilizaremos uma parametrização tal que isto ocorra em $\tau_{1}=\tau_{2}$ ). Introduziremos um corte no propagador $\left(1 /\left|x_{1}-x_{2}\right|^{2} \rightarrow 1 / \epsilon^{2}\right)$. Com isto, a contribuição divergente devido ao campo de calibre será

$$
\begin{aligned}
-\frac{g_{Y M}^{2} N}{8 \pi^{2}} \int d \tau_{1} \int_{\tau_{1}-\frac{\epsilon}{|\dot{x}|}}^{\tau_{1}+\frac{\epsilon}{|\dot{x}|}} d \tau_{2} \dot{x}^{\mu}\left(\tau_{1}\right) \dot{x}^{\nu}\left(\tau_{2}\right) \frac{\delta_{\mu \nu}}{\epsilon^{2}} & = \\
-\frac{g_{Y M}^{2} N}{8 \pi^{2}} \int d \tau_{1} \dot{x}^{\mu}\left(\tau_{1}\right) \frac{\delta_{\mu \nu}}{\epsilon^{2}}\left[x^{\nu}\left(\tau_{1}+\frac{\epsilon}{\left|\dot{x}_{1}\right|}\right)-x^{\nu}\left(\tau_{1}-\frac{\epsilon}{\left|\dot{x}_{1}\right|}\right)\right] & = \\
-\frac{g_{Y M}^{2} N}{8 \pi^{2}} \int d \tau_{1} \dot{x}^{\mu}\left(\tau_{1}\right) \frac{\delta_{\mu \nu}}{\epsilon^{2}}\left[\dot{x}^{\nu}\left(\tau_{1}\right) \frac{2 \epsilon}{\left|\dot{x}_{1}\right|}\right] & =-\frac{g_{Y M}^{2} N}{4 \pi^{2} \epsilon} \int d \tau_{1}\left|\dot{x}\left(\tau_{1}\right)\right|,
\end{aligned}
$$


sendo que na penúltima passagem expandimos $x^{\nu}\left(\tau_{1} \pm \frac{\epsilon}{\left|\dot{x}_{1}\right|}\right)$ e desprezamos termos de ordem quadrática em $\epsilon$. De forma análoga, temos que a contribuição do campo escalar é dado por

$$
\frac{g_{Y M}^{2} N}{8 \pi^{2}} \int d \tau_{1} \int_{\tau_{1}-\frac{\epsilon}{|\dot{x}|}}^{\tau_{1}+\frac{\epsilon}{|\dot{x}|}} d \tau_{2} \dot{y}^{i}\left(\tau_{1}\right) \dot{y}^{j}\left(\tau_{2}\right) \frac{\delta_{i j}}{\epsilon^{2}}=\frac{g_{Y M}^{2} N}{4 \pi^{2} \epsilon} \int d \tau_{1}\left|\dot{x}\left(\tau_{1}\right)\right| \frac{\dot{y}^{2}}{\dot{x}^{2}}
$$

Portanto, a soma da contribuição divergente de ambos os campos será

$$
\frac{g_{Y M}^{2} N}{4 \pi^{2} \epsilon} \int d \tau_{1}\left|\dot{x}\left(\tau_{1}\right)\right|\left(1-\frac{\dot{y}^{2}}{\dot{x}^{2}}\right) .
$$

Ao aplicarmos o vínculo (4.7), devido à supersimetria, vemos que esta divergência irá se cancelar. Claro que a princípio, este cálculo só nos mostra que não temos divergência no termo $C_{1}$, não em outras ordens de perturbação. Mas, como veremos mais à frente, os outros termos que irão contribuir para o cálculo de $\left\langle W_{C}\right\rangle$ serão da forma $\left(C_{1}\right)^{2 n}$, ou seja, potências deste termo, e logo vemos que pelas mesmas razões não irão gerar divergências em nossos cálculos.

Devemos notar aqui que basicamente este cancelamento se deve ao perfeito equilíbrio das contribuições dos campos escalar e de calibre, e este se deve principalmente à supersimetria. Apesar de não ser um cancelamento entre contribuições fermiônicas e bosônicas, como é usual em cálculos de supersimetria, a supersimetria neste caso implicou no vínculo (4.7), o que de fato garante o cancelamento da divergência.

\subsection{Linha reta}

Feita tais considerações quanto à primeira contribuição não nula do cálculo de $\left\langle W_{C}\right\rangle$, agora devemos fazer o cálculo das integrais que aparecem neste termo. Para isso, temos que escolher um dos contornos restritos pela supersimetria. Primeiramente, vamos considerar o caso de um contorno dado por uma linha reta. Para tal contorno, nós podemos escolher a seguinte parametrização

$$
x(\tau)=(\tau, 0,0,0), \quad-\infty \leq \tau \leq \infty .
$$


Substituindo esta parametrização em (4.13), nós temos

$$
C_{1}=\frac{g_{Y M}^{2} N}{2\left(4 \pi^{2}\right)} \int_{-\infty}^{\infty} d \tau_{1} \int_{-\infty}^{\tau_{1}} d \tau_{2} \frac{1-1}{\left|\tau_{1}-\tau_{2}\right|^{2}}=0
$$

Portanto, o valor do laço de Wilson para um contorno de uma linha reta, considerando somente a contribuição em primeira ordem em $\lambda$, será dado somente por:

$$
\left\langle W_{\text {reta }}\right\rangle=1 \text {. }
$$

\subsection{Laço circular}

O segundo contorno possível, que preserva a metade das supercargas, é o contorno circular, obtido por uma transformação de inversão da trajetória reta. Para o caso de um contorno circular, nós podemos usar a seguinte parametrização

$$
x(\tau)=(\cos \tau, \sin \tau, 0,0), \quad 0 \leq \tau \leq 2 \pi
$$

Com esta parametrização, temos que o valor de $C_{1}$ será

$$
C_{1}=\frac{g_{Y M}^{2} N}{2\left(4 \pi^{2}\right)} \int_{0}^{2 \pi} d \tau_{1} \int_{0}^{\tau_{1}} d \tau_{2} \frac{1-\sin \tau_{1} \sin \tau_{2}-\cos \tau_{1} \cos \tau_{2}}{\left(\cos \tau_{1}-\cos \tau_{2}\right)^{2}+\left(\sin \tau_{1}-\sin \tau_{2}\right)^{2}}=\frac{g_{Y M}^{2} N}{2 ! 4}
$$

Portanto, obtemos um fator de $g_{Y M}^{2} N / 4$ por conta das contrações dos índices e o cálculo do traço, enquanto que obtemos um fator de 2 ! pela integração em $\tau_{1}$ e $\tau_{2}$. O propagador combinado é então costante e não depende de $x$ ou $y$.

É interessante notar que, apesar do contorno poder ser obtido através de uma transformação conforme da trajetória reta, o resultado de $C_{1}$ é distinto para o caso de um contorno circular. Porém, esta diferença entre os resultados não representa uma inconsistência, uma vez que este fato pode ser entendido como sendo causado por efeitos de uma anomalia conforme [26].

\subsubsection{Soma dos gráficos planares}

Como foi discutido na Seção 3.1.3, sabemos que para o limite de $N$ grande, com $g_{Y M}^{2} N$ fixo, somente gráficos planares serão relevantes. Apesar da grande simplificação de considerarmos diagramas planares, ainda nos falta considerarmos as contribuições de vértices 
e loops para cada ordem em $g_{Y M}$. Porém, foi verificado em [25] que em primeira ordem a contribuição do vértice se cancela exatamente com a contribuição da correção de loop, Figura (4.2). ${ }^{5}$ Este cancelamento, em ordem dominante, entre o termo de vértice e de loop, sugeriu a especulação de que as únicas contribuições relevantes seriam daquelas sem correções de vértices ou loops, uma vez que estas se cancelariam mutuamente em todas as ordens. A princípio, tal especulação, apesar de não ser verificada, permitiu o cálculo dos laços de Wilson em questão, que, de forma muito intrigante, coincidia com o resultado obtido na supergravidade, utilizando a correspondência AdS/CFT. Tal fato contribuiu para expectativa da confirmação desta especulação. De fato, anos mais tarde, através do trabalho de [12], que será abordado na Seção 5.1, foi possível estabelecer de modo formal e mais geral a relação do resultado dos diagramas planares sem vértices, que culmina no cálculo de modelo de matrizes abordado no Capítulo 5, e o resultado exato dos laços de Wilson supersimétricos.

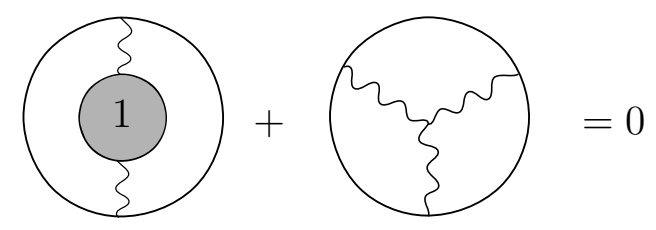

Figura 4.2: Cancelamento dos termos de vértices e correções a um loop do propagador.

Portanto, devido às razões apresentadas, assumiremos o fato de que as correções de loops se cancelam com termos de vértices, e assim daremos continuidade ao cálculo do $\left\langle W_{C}\right\rangle$ desconsiderando tais termos. Logo, fazendo tal simplificação nós teremos apenas diagramas que chamaremos de 'rainbows' ou 'ladders', representados na Figura 4.3, cujo cálculo será elaborado a seguir.

Vamos análisar um termo planar genérico desta expansão. Primeiramente, sabemos que, pelo teorema de Wick, somente os termos de ordem par da expansão do laço vão contribuir (uma vez que precisamos fazer a contração dois a dois dos campos gerados pela expansão) da onde podemos ver que teremos diagramas proporcionais à $\left(g_{Y M}^{2}\right)^{n}$. Desta

\footnotetext{
${ }^{5}$ Tais diagramas não devem ser confundidos com diagramas de Feynman usuais do tipo "bolhas do vácuo"
} 

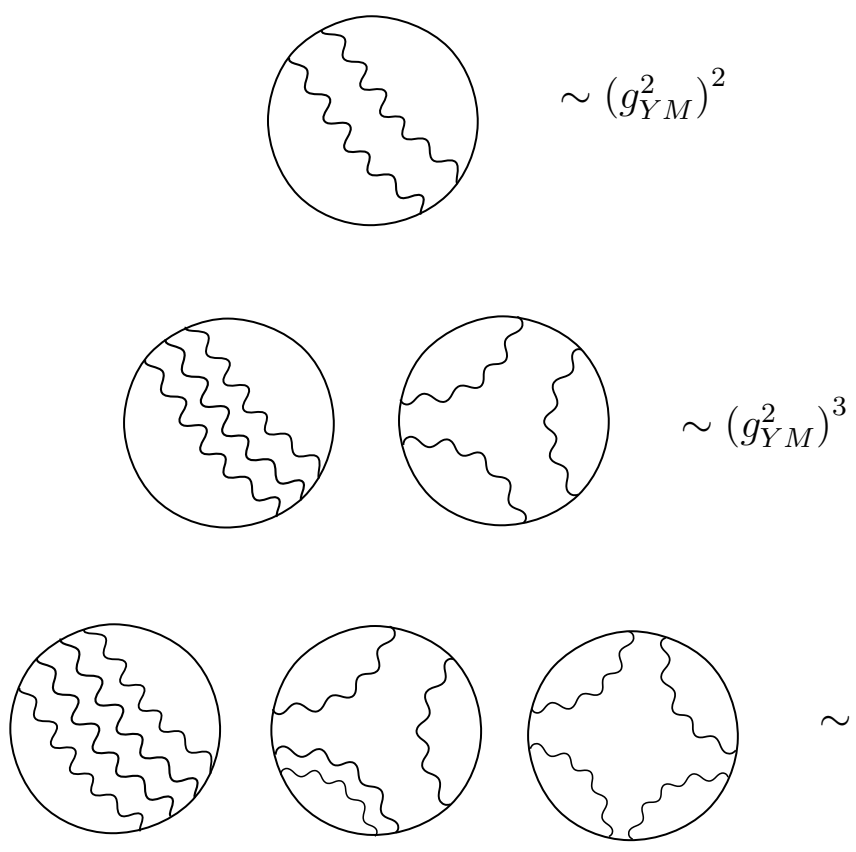

$$
\sim\left(g_{Y M}^{2}\right)^{4}
$$

Figura 4.3: Diagramas 'rainbow' ou 'ladders', cada diagrama com $n$ propagadores será porporcional à $\left(g_{Y M}^{2}\right)^{n}$.

observação, temos que um termo genérico, não nulo, da expansão será da forma

$\frac{1}{N} \int_{0}^{2 \pi} d \tau_{1} \int_{0}^{\tau_{1}} d \tau_{2} \ldots \int_{0}^{\tau_{2 n-1}} d \tau_{2 n} \operatorname{Tr}\left\langle\left(i \dot{x}_{1} \cdot A_{1}+\left|\dot{x}_{1}\right| \theta \cdot \Phi_{1}\right) \ldots\left(i \dot{x}_{2 n} \cdot A_{2 n}+\left|\dot{x}_{2 n}\right| \theta \cdot \Phi_{2 n}\right)\right\rangle$.

Aqui $A_{1}^{\mu} \equiv A^{\mu}\left(x_{1}\right), \ldots$, e o fator de $1 /(2 n)$ !, devido à expansão da exponencial, foi cancelado pelo fator que surge devido à escolha do ordenamento temporal particular das integrais (2.11).

Agora, devemos calcular todas as contrações de Wick que representam termos planares. Fazendo todas as possíveis contrações que resultam em diagramas do tipo rainbow, dado um $n$ fixo, vemos que teremos alguns diagramas equivalentes através de permutações dos $x_{i}$. Além destes, também teremos outros diagramas com distintas topologias para o mesmo valor de $n$, como os diagramas colineares da Figura 4.3. Podemos ver que qualquer um destes termos pode ser organizado como produtos de termos semelhantes à $C_{1}$, ou seja, 
termos da forma

$$
\left\langle\left(i \dot{x}_{i} \cdot A^{a}\left(\tau_{i}\right)+\left|\dot{x}_{i}\right| \theta \cdot \Phi^{a}\left(\tau_{i}\right)\right)\left(i \dot{x}_{j} \cdot A^{b}\left(\tau_{j}\right)+\left|\dot{x}_{j}\right| \theta \cdot \Phi^{b}\left(\tau_{j}\right)\right)\right\rangle=\frac{g_{Y M}^{2} \delta^{a b}}{8 \pi^{2}} .
$$

Além disso, a contribuição de um dado diagrama não depende da forma com que desenhamos os propagadores, de como ligamos os pontos $x_{i}$. Isto faz com que os diagramas sejam independentes da permutação entre os $x_{i}$ ou mesmo da topologia, ou seja, para um mesmo $n$, cada um desses contribuirão com a mesma quantidade.

A quantidade com que um diagrama com um dado $n$ fixo irá contribuir é dada pela seguinte análise: O fator de $\delta^{a b}$ irá contrair os geradores $T^{a}$ dois a dois, e usando repetidamente a identidade $T^{a} T^{a}=\frac{N}{2}$, nos dará um fator de $N / 2$ para cada contração . Tomando o traço da identidade, teremos o fator de $N$, que cancelará o mesmo no denominador de (4.22). Lembrando do resultado (4.21), vemos que teremos um fator de $(2 n)$ ! no denominador, devido as integrações em $\tau_{i}$. Portanto, qualquer diagrama rainbow de ordem $n$ da expansão do laço de Wilson irá contribuir com $\left(g_{Y M}^{2} N / 4\right)^{n} /(2 n)$ !.

Logo, a contribuição total para um dado $n$ fixo é obtida simplesmente multiplicando a contribuição genérica de um diagrama de ordem $n$ pelo número de diagramas que podem ser construídos da forma rainbow. Portanto, a contribuição de ordem $\lambda^{n}$ é dada por

$$
\left.\frac{\left(g_{Y M}^{2} N / 4\right)^{n}}{(2 n) !} \times \text { (número de diagramas rainbow com } n \text { propagadores }\right) .
$$

Aqui, vemos que, basicamente, o problema de se calcular o VEV do laço de Wilson supersimétrico é resumido à contagem do número de possíveis diagramas rainbow que podem ser construídos com um dado número de propagadores fixos. Apesar de não intuitivo, foi notado em [25] que o cálculo do número de diagramas rainbow com $n$ propagadores, que denotaremos por $A_{n}$, pode ser obtido através de modelo de matrizes, estudado no próximo capítulo. Tal relação entre o cálculo feito até aqui e os modelos de matrizes é não trivial e a demonstração formal original deste fato consta em [12]. Uma explicação de como esta relação se dá será vista na Seção 5.1. 


\section{Capítulo 5}

\section{Resultados exatos via modelos de}

matrizes

Como dito na seção anterior, esta relação entre a contagem do número de diagramas planares e o modelo de matrizes possui uma natureza muito mais geral. Como foi demonstrado em [12], é possível estabelecer uma relação formal entre os operadores supersimétricos feitos no contexto da $\mathcal{N}=4$ super Yang-Mills e operadores equivalentes de modelos de matrizes. Tal conjectura a princípio foi motivada pelo fato de que a contagem dos diagramas rainbow levava a uma função que era conhecida em modelo de matrizes como distribuição de autovalores. Além disso, objetos como $C_{1}$ em (4.21) resultarem, de forma não trivial, em quantidades que são independentes das coordenadas do espaço-tempo, ou seja propagadores constantes, indicava uma possível relação com um problema em zero dimensões. Iniciaremos este capítulo fazendo uma revisão não muito detalhada da prova de Pestun e a seguir vamos introduzir o modelo de matrizes em zero dimensões, definindo de forma geral a função de partição da teoria e reescrevendo-a em termos de seus autovalores. Em seguida, faremos o cálculo de $A_{n}$ através de dois métodos distintos, utilizando a aproximação por ponto de sela e o método dos polinômios ortogonais. 


\subsection{Prova de Pestun via localização}

Como dito antes, a conjectura do trabalho de [25], em que se supõe a equivalência do cálculo dos laços supersimétricos através da teoria de campos e o cálculo feito no modelo de matrizes, foi demonstrada no trabalho [12]. Por conta da dificuldade do nível técnico requerido para uma revisão completa e detalhada deste, aqui vamos fazer uma revisão simplificada deste argumento, somente explicando as idéias básicas.

Temos que a nossa ação é invariante pelas cargas supersimétricas

$$
Q S[X]=0
$$

sendo que por $X$ estamos denotando todos os campos que aparecem na ação. Agora vamos fazer uma deformação desta ação da forma

$$
S[X] \rightarrow S+t Q V[X]
$$

onde $t$ é um parâmetro livre e $V[X]$ é um funcional que depende dos campos, cujas propriedades definiremos. Podemos construir $V[X]$ possuindo as seguintes propriedades:

$$
\begin{aligned}
& Q^{2} V[X]=0 \\
& Q V[X] \geq 0 .
\end{aligned}
$$

Assim, podemos escrever a nossa função de partição como sendo

$$
Z=\int \mathcal{D} X e^{-(S[x]+t Q V[X])}
$$

Derivando com respeito ao parâmetro $t$

$$
\begin{aligned}
\frac{d Z}{d t} & =\int \mathcal{D} X(Q V) e^{-(S[x]+t Q V[X])} \\
& =\int \mathcal{D} X Q\left(V e^{-(S[x]+t Q V[X])}\right)-\int \mathcal{D} X V Q\left(e^{-(S[x]+t Q V[X])}\right)
\end{aligned}
$$

No primeiro termo encontramos um termo de superfície, enquanto o segundo é nulo pela invariância da ação e pela construção de $V[X]$ (5.3). Portanto temos que:

$$
\frac{d Z}{d t}=0
$$


ou seja, $Z$ não depende do parâmetro de deformação $t$. Em particular podemos escolher $t=0$ e vemos que a função de partição deformada é igual à original. Podemos ver que o mesmo argumento vale para o cálculo do VEV de operadores supersimétricos, tais que $Q W=0$

$$
\begin{aligned}
\frac{d\langle W\rangle}{d t} & =\int \mathcal{D} X(Q V)\left(e^{-(S[x]+t Q V[X])} W\right) \\
& =\int \mathcal{D} X Q\left(V e^{-(S[x]+t Q V[X])} W\right)-\int \mathcal{D} X V Q\left(e^{-(S[x]+t Q V[X])} W\right),
\end{aligned}
$$

sendo que o segundo termo é nulo pelos mesmos argumentos anteriores somados à invariância supersimétrica de $W$. Portanto, nós temos que VEV's também não possuem dependência do parâmetro $t$.

Vamos agora analisar o que acontece quando tomamos o limite de $t \rightarrow \infty$ no caso da função de partição, sendo que o mesmo vale para VEV's de operadores supersimétricos

$$
Z=\lim _{t \rightarrow \infty} \int \mathcal{D} X e^{-S[x]-t Q V[X]} .
$$

Vemos que neste limite, somente as configurações em que $Q V[X] \rightarrow 0$ contribuem de forma não nula. Assim, temos que a integral é localizada, ou seja, seu domínio de integração é restringido pelas configurações dos campos que correspondem à condição

$$
Q V[X]=0 .
$$

Procurando as configurações dos campos que satisfaçam tal condição, nós somos levados a uma condição que escreveremos de forma esquemática ${ }^{1}$

$$
Q V[X] \sim \alpha\left(F_{\mu \nu}\right)^{2}+\beta\left(\Phi^{i \neq 9}\right)^{2}+\gamma\left(D_{\mu} \Phi^{i}\right)^{2}+\ldots,
$$

onde as reticências indicam outros termos que não serão importantes para a análise e $\alpha$, $\beta$ e $\gamma$ são constantes positivas. Desta condição nós podemos concluir que:

$$
Q V[X]=0 \quad \Rightarrow \quad \begin{cases}A_{\mu}=0, & \mu=0, \ldots, 3 \\ \Phi^{i}=0, & i=4, \ldots, 8 \\ \Phi^{9}=M, & \text { (matriz constante) }\end{cases}
$$

\footnotetext{
${ }^{1}$ Mais detalhes destas condições podem encontrados em [12].
} 
Portanto, nós vemos que para cálculo de VEV's de operadores supersimétricos nós podemos utilizar o argumento acima, uma vez que o mesmo resultado deve valer para uma ação não deformada.

A princípio, este resultado nos dá uma ação trivialmente nula, se utilizarmos a ação (3.33) em quatro dimensões no espaço plano. Porém no trabalho original de [12] a teoria é definida em uma esfera $S^{4}$, e não em $\mathbb{R}^{1,3}$ como vimos na Seção 3.3 (dada a invariância conforme da teoria isto é possível). Uma vez feito isto, as contribuições dos ínstantons que a priori podem contribuir para a função de partição, são localizados nos polos da esfera de modo a cancelarem-se mutuamente. Além de simplificar o cálculo dos instantons, definir a teoria sobre $S^{4}$ causa uma deformação, devido à curvatura não nula do espaço, do termo cinético da forma $(\partial \Phi)^{2} \rightarrow(\partial \Phi)^{2}+\frac{R}{6} \Phi^{2}$, onde aqui $R$ é o escalar de curvatura (este termo também é chamado de massa conforme).

Logo, devido ao termo quadrático em $\Phi$, a imposição das condições (5.12) resulta em uma ação não nula da forma

$$
\left.S\right|_{S^{4}} \rightarrow \frac{k}{g_{Y M}^{2}} \operatorname{Tr}(M)^{2}
$$

onde $k$ aqui é uma constante que pode ser definida tal que esta possa ser identificada exatamente com a ação proposta por [25], ou seja, a ação zero dimensional do modelo de matrizes vista na introdução.

Portanto podemos compreender, através dos argumentos dados aqui, que de fato o cálculo dos laço de Wilson supersimétrico em questão resulta no cálculos de modelos de matrizes. Mais precisamente, resultam em modelo de matrizes cujo potencial é gaussiano e portanto sem termos de loops e vértices, como foi conjecturado em [25].

\subsection{Modelo de matrizes}

Motivados pelo nosso problema de calcular $A_{n}$, nós iremos considerar um modelo em zero dimensões, ou seja, sem dependências das coordenadas do espaço tempo. Tais modelos podem ser vistos como sendo os modelos mais simples de teorias de calibre, onde os campos são representados por matrizes hermitianas $N \times N$, que denotaremos por $M$, independentes do espaço-tempo, ou seja, cada uma de suas entradas são constantes em relação ao espaço- 
tempo. Tais modelos podem ser definidos a partir de uma ação da forma ${ }^{2}$

$$
\frac{1}{g_{Y M}^{2}} S(M)=\frac{1}{2 g_{Y M}^{2}} \operatorname{Tr} M^{2}+\frac{1}{g_{Y M}^{2}} \sum_{p \geq 3} \frac{g_{p}}{p} \operatorname{Tr} M^{p},
$$

onde $g_{p}$ é uma nova constante de acoplamento.

Observamos que esta ação não possui termos cinéticos, por conta de que $\partial_{\mu} M=0$, uma das simplificações devida ao fato do modelo ser zero dimensional. Para fazer os cálculos dos laços de Wilson na $\mathcal{N}=4$ super Yang-Mills, nós utilizaremos apenas o primeiro termo da ação, chamado de termo quadrático ou gaussiano, apesar do tratamento a seguir possuir uma generalização para o caso de uma ação com $g_{p} \neq 0$.

A partir desta ação, podemos escrever a função de partição da teoria

$$
Z=\frac{1}{\operatorname{Vol}(U(N))} \int d M e^{-\frac{1}{g_{Y M}^{2}} S(M)}
$$

sendo que $U(N)$ é o grupo unitário responsável pelas transformações de calibre que as matrizes $M$ podem sofrer, e $\operatorname{Vol}(U(N))$ é o fator de normalização usual devido ao fixamento do calibre a ser utilizado. Quanto à medida que estamos considerando, vamos utilizar a medida de Haar

$$
d M=\prod_{i=1}^{N} d M_{i i} \prod_{1 \leq i \leq N} d \operatorname{Re}\left(M_{i j}\right) d \operatorname{Im}\left(M_{i j}\right),
$$

que podemos ver como sendo uma medida análoga às "integrais de trajetória", considerando cada grau de liberdade que as matrizes $M$ possuem como os campos a serem integrados. Uma das importantes propriedades da medida de Haar é a invariância sobre as transformações de calibre, da mesma forma que a ação (5.14) também é, ou seja, invariância pela transformação

$$
M \rightarrow U M U^{\dagger}=M^{U}
$$

Em nosso caso, podemos definir o modelo de matrizes gaussiano como sendo

$$
Z_{G}=\frac{1}{\operatorname{Vol}(U(N))} \int d M e^{-\frac{1}{2 g_{Y M}^{2}} \operatorname{Tr} M^{2}} .
$$

Da mesma forma como usualmente fazemos em teorias quânticas de campos, vamos calcular VEV's de funcionais $f(M)$ através de

$$
\langle f(M)\rangle=\frac{\int d M f(M) e^{-\frac{1}{2 g_{Y M}^{2}} \operatorname{Tr} M^{2}}}{\int d M e^{-\frac{1}{2 g_{Y M}^{2}} \operatorname{Tr} M^{2}} .}
$$

\footnotetext{
${ }^{2}$ Revisões podem ser encontradas em [27, 28].
} 
Para simplificarmos as contas, gostaríamos de usar a invariância de calibre para escrever as matrizes $M$ da forma diagonal, ou seja, em termos de seus autovalores

$$
\left(M^{D}\right)_{i j}=\lambda_{i} \delta_{i j}
$$

sendo que nesta equação, não estamos utilizando a notação de soma de Einstein para índices repetidos. Desta forma, poderemos escrever as integrais como

$$
I_{N} \equiv \int d M F(M) \rightarrow \int \prod_{i=1}^{N} d \lambda_{i} F\left(M^{D}\right) J\left(M^{D}\right)
$$

sendo que por $F\left(M^{D}\right)$, estamos denotando uma função invariante, $F\left(M^{D}\right)=F(M)$, em nosso caso de interesse $F(M)=f(M) e^{-\frac{1}{2 g_{Y M}^{2}} \operatorname{Tr} M^{2}}$.

Para simplificarmos as equações desta forma, nós iremos seguir um procedimento equivalente à do Faddeev-Popov, utilizando a simetria de calibre para escrever as matrizes em termos de seus autovalores. Para isto, primeiramente vamos introduzir o seguinte objeto

$$
\Delta^{-1}(M) \equiv \int d U \prod_{1 \leq i<j \leq N} \delta^{(2)}\left[\left(M^{U}\right)_{i j}\right]
$$

onde $d U$ é a medida de Haar sobre o grupo unitário e as funções $\delta^{(2)}$ denotam

$$
\delta^{(2)}\left[\left(M_{i j}^{U}\right)\right]=\delta\left[\operatorname{Re}\left(U M U^{\dagger}\right)_{i j}\right] \delta\left[\operatorname{Im}\left(U M U^{\dagger}\right)_{i j}\right]
$$

Como a medida $d U$ é invariante, segue que $\Delta(M)=\Delta\left(M^{U}\right)$. Agora, iremos introduzir este objeto no lado esquerdo da (5.21) para obter

$$
I_{N}=\int d M F(M) \Delta(M) \int d U \prod_{1 \leq i<j \leq N} \delta^{(2)}\left[\left(M_{i j}^{U}\right)\right]
$$

Podemos agora utilizar a invariância dos objetos $F(M), d M$ e $\Delta(M)$ para poder trocar o $\operatorname{argumento}$ da $\delta^{(2)}, M \rightarrow M^{U^{-1}}$ e obter

$$
\int d U \int d M F(M) \Delta(M) \prod_{1 \leq i<j \leq N} \delta^{(2)}\left(M_{i j}\right)=\Omega_{N} \int \prod_{i=1}^{N} d \lambda_{i} F\left(M^{D}\right) \Delta\left(M^{D}\right)
$$

sendo que aqui definimos

$$
\Omega_{N}=\int d U
$$


Comparando com (5.21), podemos identificar

$$
J\left(M^{D}\right)=\Omega_{N} \Delta\left(M^{D}\right)
$$

Agora, precisamos calcular o valor de $\Delta\left(M^{D}\right)$ e $\Omega_{N}$ para obtermos $J\left(M^{D}\right)$ e finalmente escrevermos a expressão explícita de (5.21). Notemos, porém, que de fato o fator $\Omega_{N}$ será cancelado na normalização do valor esperado de qualquer funcional e, portanto, não será relevante. Primeiramente, vamos calcular $\Delta\left(M^{D}\right)$. Para isso devemos escrever a matriz unitária $U$ próxima da identidade, em termos de matrizes $A$ infinitesimais e anti-hermitianas ${ }^{3}$

$$
U=e^{A}=1+A+\mathcal{O}\left(A^{2}\right)
$$

Desta forma, a função delta $\delta^{(2)}\left[\left(M_{i j}^{U}\right)\right]$ implica em

$$
\left(e^{A} M^{D} e^{-A}\right)_{i j}=0 \quad \text { para } i \neq j
$$

e utilizando a expansão (5.28) temos que, desprezando termos de segunda ordem em $A$, a condição acima implica em

$$
\sum_{k, l} \lambda_{i} \delta_{i j}-\lambda_{i} \delta_{i k} A_{k j}+A_{i l} \lambda_{l} \delta_{l j}=\left(M^{D}\right)_{i j}+\left[A, M^{D}\right]_{i j}=0, \quad \text { para } i \neq j
$$

ou seja,

$$
\lambda_{i} A_{i j}=\lambda_{j} A_{i j}, \quad \text { para cada } i \neq j .
$$

Como queremos que as matrizes $M^{D}$ não sejam múltiplas da identidade, queremos que cada autovalor seja de fato uma variável de integração independente e temos que ter a condição $A_{i j}=0$ para $i \neq j$. Agora, utilizaremos esta condição para reescrever a função delta $\delta^{(2)}\left[\left(M_{i j}^{U}\right)\right]$ em termos das variáveis $\lambda_{i}$, fazendo uso da propriedade da função delta

$$
\delta[F(x)]=\sum_{i} \frac{\delta\left(x-x_{i}\right)}{\left|F^{\prime}\left(x_{i}\right)\right|},
$$

onde $x_{i}$ são os zeros da função $F(x)$. Reescrevendo o argumento da delta, nós podemos desprezar o primeiro termo da (5.30), que satisfaz trivialmente a condição, ficando com

$$
\left(e^{A} M^{D} e^{-A}\right)_{i j}=\left[A, M^{D}\right]_{i j}+\mathcal{O}\left(A^{2}\right)=A_{i j}\left(\lambda_{j}-\lambda_{i}\right)+\mathcal{O}\left(A^{2}\right) .
$$

\footnotetext{
${ }^{3}$ Aqui escolhemos matrizes anti-hermitianas, ao invés de matrizes hermitianas, para simplesmente não carregarmos um fator multiplicativo $i$ (como em $U=1+i A)$.
} 
Finalmente, calculamos explicitamente o valor de $\Delta^{-1}\left(M^{D}\right)$

$$
\begin{aligned}
\Delta^{-1}\left(M^{D}\right) & =\int \prod_{1 \leq i<j \leq N} d\left(\operatorname{Re} A_{i j}\right) d\left(\operatorname{Im} A_{i j}\right) \prod_{1 \leq i<j \leq N} \delta^{(2)}\left[A_{i j}\left(\lambda_{j}-\lambda_{i}\right)+\ldots\right] \\
& =\left[\prod_{1 \leq i<j \leq N}\left(\lambda_{j}-\lambda_{i}\right)^{2}\right]^{-1}
\end{aligned}
$$

Portanto

$$
\Delta\left(M^{D}\right)=\prod_{1 \leq i<j \leq N}\left(\lambda_{j}-\lambda_{i}\right)^{2}
$$

o que nos dá

$$
I_{N}=\Omega_{N} \int \prod_{i=1}^{N} d \lambda_{i} F\left(M^{D}\right) \prod_{1 \leq i<j \leq N}\left(\lambda_{j}-\lambda_{i}\right)^{2} .
$$

Quanto ao valor de $\Omega_{N}$, podemos fazer da seguinte forma. Primeiro podemos calcular diretamente o valor de $I_{N}$ no caso Gaussiano

$$
\int d M e^{-\frac{1}{2 g_{Y M}^{2}} \sum_{i=1}^{N} \lambda_{i}^{2}}=\left(2 \pi g_{Y M}^{2}\right)^{N^{2} / 2},
$$

mas também podemos calcular o valor de $I_{N}$ pela (5.36). Substituindo a expressão explícita $F\left(M^{D}\right) \rightarrow e^{-\frac{1}{2 g_{Y M}^{2}} \sum_{i=1}^{N} \lambda_{i}^{2}}$ e utilizando métodos como o de polinômios ortogonais, que veremos mais adiante, temos que

$$
I_{N}=\Omega_{N} g_{Y M}^{N^{2}}(2 \pi)^{N / 2} G_{2}(N+2),
$$

sendo $G_{2}(z)$ a função de Barnes, definida por

$$
G_{2}(z+1)=\Gamma(z) G_{2}(z), \quad G_{2}(1)=1
$$

Comparando os dois resultados, nós podemos concluir que

$$
\Omega_{N}=\frac{(2 \pi)^{\frac{N(N-1)}{2}}}{G_{2}(N+2)}
$$

e portanto nós podemos escrever a nossa função de partição escolhendo

$$
\operatorname{vol}(U(N))=\frac{(2 \pi)^{\frac{N(N+1)}{2}}}{G_{2}(N+1)},
$$

obtendo a forma de $Z$ que vamos utilizar para os nossos cálculos

$$
Z=\frac{1}{N !} \frac{1}{(2 \pi)^{N}} \int \prod_{i=1}^{N} d \lambda_{i} \prod_{1 \leq i<j \leq N}\left(\lambda_{j}-\lambda_{i}\right)^{2} e^{-\frac{1}{2 g_{Y M}^{2}} \sum_{i=1}^{N} \lambda_{i}^{2}}
$$




\subsection{Aproximação por ponto de sela}

Nesta seção, vamos implementar um dos métodos utilizados no cálculo de modelos de matrizes, o chamado método da aproximação por ponto de sela. Este método possui uma aplicabilidade mais geral, comparado ao que será desenvolvido na próxima seção, por poder ser aplicado em diversões tipos de potências, não somente no nosso caso gaussiano. Em contrapartida, este método possui a limitação de ser uma aproximação, como o próprio nome nos diz. Esta aproximação é utilizada logo no início do método, fazendo uso do limite de $N \rightarrow \infty$ e, portanto, não nos dando um resultado exato.

Primeiramente, para implementarmos tal método, a partir da (5.42), vamos definir uma ação efetiva da forma

$$
S_{e f}\left(\lambda_{i}\right)=-\frac{1}{\lambda N} \sum_{i=1}^{N} V\left(\lambda_{i}\right)+\frac{2}{N^{2}} \sum_{i<j} \ln \left|\lambda_{i}-\lambda_{j}\right|,
$$

sendo que em nosso caso $V\left(\lambda_{i}\right)=\frac{1}{2} \lambda_{i}^{2}$. Em termos desta ação efetiva nós podemos escrever a função de partição da seguinte forma

$$
Z=\frac{1}{N !} \int \prod_{i=1}^{N} \frac{d \lambda_{i}}{2 \pi} e^{N^{2} S_{e f}\left(\lambda_{i}\right)} .
$$

Agora, vamos olhar para o limite de $N$ grande. Para $N$ grande, temos que a ação efetiva deve ser de ordem $\mathcal{O}(1)$, dado que as somas dos autovalores deve ser de ordem $\mathcal{O}(N)$. Portanto, se tomarmos este limite, nós temos que o fator de $N$ multiplicando a ação efetiva na exponencial irá provocar uma queda abrupta (ou uma oscilação de alta frequência no caso de uma fase imaginária, mas que gera o mesmo efeito). Mas, se pensarmos em $1 / N^{2}$ sendo algo como $\hbar$, nós sabemos que a integração será dominada pela configuração dos pontos de sela da ação efetiva. Em outras palavras, sabemos que a configuração dos autovalores que irá contribuir respeitará a equação de ponto de sela, que pode ser obtida através da variação da ação efetiva com relação a um de seus autovalores:

$$
\frac{1}{2 \lambda} V^{\prime}\left(\lambda_{i}\right)=\frac{1}{N} \sum_{j, j \neq i} \frac{1}{\lambda_{i}-\lambda_{j}}, \quad i=1, \ldots, N .
$$

Tal equação vai definir a distribuição de autovalores a partir do potencial $V^{\prime}\left(\lambda_{i}\right)$. Podemos 
pensar nesta distribuição sendo da forma

$$
\rho(\xi)=\frac{1}{N} \sum_{i=1}^{N} \delta\left(\xi-\lambda_{i}\right)
$$

sendo que os $\lambda_{i}$ são soluções de (5.45). Uma forma interessante para pensarmos neste problema de forma mais intuitiva é pensar em um sistema clássico mecânico, composto por $N$ partículas, cada uma delas sujeita a um potencial da forma

$$
V_{e f}\left(\lambda_{i}\right)=V\left(\lambda_{i}\right)-\frac{2 \lambda}{N} \sum_{i<j} \ln \left|\lambda_{i}-\lambda_{j}\right|
$$

onde temos um termo de potencial ordinário de fundo e mais um termo de interação entre as partículas, que podemos pensar como sendo um tipo de uma interação coulombiana. Por conta do termo de interação, sabemos que os mínimos do potencial serão modificados. Para $\lambda$ pequenos o potencial ordinário irá ter mais relevância para a disposição dos mínimos do potencial efetivo, enquanto que para um $\lambda$ grande temos que o termo de repulsão irá espalhá-las.

Para o limite de $N$ grande esperamos que esta distribuição (5.46) se torne uma função continua dentro de um suporte compacto que denotaremos por $\mathcal{I}$. Nosso objetivo agora é resolver a equação de ponto de sela e encontrar esta distribuição. Para isto vamos definir uma função auxiliar que chamaremos de resolvente, com a qual poderemos escrever a solução de $\rho(\xi)$ :

$$
\omega(z)=\int \frac{\rho(\xi) d \xi}{\xi-z}=\frac{1}{N} \sum_{i=1}^{N} \frac{1}{\lambda_{i}-z} .
$$

Podemos escrever a equação de ponto de sela em termos do resolvente multiplicando a (5.45) por $1 /\left(\lambda_{i}-z\right)$ e somando sobre $i$. Fazendo isso podemos obter

$$
\omega^{2}(z)-\frac{1}{N} \omega^{\prime}(z)+\frac{1}{\lambda} V^{\prime}(z) \omega(z)=-\frac{1}{N \lambda} \sum_{i} \frac{V^{\prime}(z)-V^{\prime}\left(\lambda_{i}\right)}{z-\lambda_{i}} .
$$

Neste ponto, vamos efetivamente tomar o limite de $N$ grande. Como consequência, nós podemos desprezar o termo de $\omega^{\prime}(z)$. Neste limite, vamos obter uma distribuição contínua de autovalores, como havíamos dito antes. Além disso, vamos impor a condição de normalização

$$
\int \rho(\xi) d \xi=1
$$


Dentro deste limite, nós podemos escrever a equação de ponto de sela (5.45) na forma contínua

$$
2 f \frac{\rho\left(\xi^{\prime}\right) d \xi^{\prime}}{\xi-\xi^{\prime}}=\frac{1}{\lambda} V^{\prime}(\xi)
$$

onde a integral acima denota o valor principal de Cauchy. De forma equivalente podemos escrever a equação de descontinuidade (para mais detalhes ver o Apêndice III)

$$
\lim _{\epsilon \rightarrow 0^{+}} \omega(z+i \epsilon)+\lim _{\epsilon \rightarrow 0^{+}} \omega(z-i \epsilon)=-\frac{1}{\lambda} V^{\prime}(z) .
$$

No limite de $N$ grande temos que a equação (5.49) pode ser escrita como

$$
\omega^{2}(z)+\frac{1}{\lambda} V^{\prime}(z) \omega(z)+\frac{1}{4 \lambda^{2}} R(z)=0
$$

onde definimos

$$
R(z)=4 \lambda \int d \xi \rho(\xi) \frac{V^{\prime}(z)-V^{\prime}(\xi)}{z-\xi}
$$

O que temos que fazer agora é resolver a equação (5.53), que é uma equação de segundo grau para $\omega(z)$. Resolvendo esta equação, chegamos a uma solução da forma

$$
\omega(z)=\frac{1}{2 \lambda}\left(-V^{\prime}(z)+\sqrt{\left(V^{\prime}(z)\right)^{2}-R(z)}\right)
$$

$\mathrm{Ou}$

$$
\omega(z)=\frac{1}{2 \lambda}\left(-V^{\prime}(z)+\sigma(z)\right)
$$

onde $\sigma(z)$ é a parte singular da função $\omega$ (a menos da normalização)

$$
\sigma(z)=\sqrt{\left(V^{\prime}(z)\right)^{2}-R(z)} .
$$

Observamos que ainda não temos uma forma explícita do resolvente $\omega(z)$, uma vez que escrevemos este em termos de $R(z)$ que por sua vez depende de $\rho(\xi)$ [28]. Para obtê-lo, em geral, temos que procurar soluções de funções com um corte de descontinuidade e impor as condições assintóticas para o resolvente, além da normalização de $\rho(\xi)$. Em nosso caso, uma vez que $V(z)=z^{2} / 2$, nós não precisaremos nos preocupar com a dependência de $R(z) \operatorname{com} \rho(z)$

$$
R(z)=4 \lambda \int d \xi \rho(\xi) \frac{z-\xi}{z-\xi}=4 \lambda
$$


onde usamos a normalização $\int \rho(\xi) d \xi=1$. Substituindo este resultado na expressão do resolvente, chegamos a

$$
\omega(z)=\frac{1}{2 \lambda}\left[-z+\sqrt{z^{2}-4 \lambda}\right]
$$

Lembrando que $\omega(z)$ é descrito em termos de $\rho$, nós podemos inverter a relação e escrever $\rho$ em termos do resolvente

$$
\rho(z)=\lim _{\epsilon \rightarrow 0^{+}} \frac{1}{2 \pi i}[\omega(z+i \epsilon)-\omega(z-i \epsilon)] .
$$

Uma vez calculado, o resultado para a distribuição de autovalores é

$$
\rho(z)=\frac{1}{2 \pi \lambda} \sqrt{4 \lambda-z^{2}} .
$$

Fazendo um gráfico desta função vemos que esta forma uma semicírculo de raio $2 \sqrt{\lambda}$, Figura 5.1, a famosa lei do semicírculo de Wigner-Dyson.

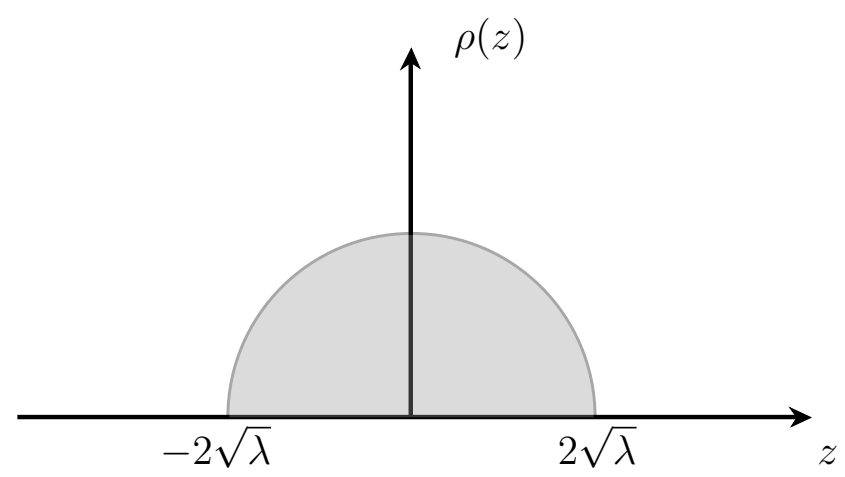

Figura 5.1: Gráfico da distribuição de autovalores do modelo de matrizes gaussiano, a lei do semicírculo de Wigner-Dyson.

Daqui, também podemos ver que $\mathcal{I}=[-2 \sqrt{\lambda}, 2 \sqrt{\lambda}]$. Utilizando esta distribuição de autovalores podemos calcular o valor esperado de objetos como

$$
\frac{1}{N}\left\langle\operatorname{Tr} M^{n}\right\rangle=\int_{\mathcal{I}} d \xi \xi^{n} \rho(\xi)=\int_{\mathcal{I}} d \xi \xi^{n} \frac{1}{2 \pi \lambda} \sqrt{4 \lambda-\xi^{2}},
$$

e portanto temos que

$$
\begin{aligned}
\left\langle\frac{1}{N} \operatorname{Tr} \exp (M)\right\rangle & =\sum_{n} \frac{1}{n !} \frac{1}{N}\left\langle\operatorname{Tr} M^{n}\right\rangle \\
& =\int_{\mathcal{I}} d \xi \frac{e^{\xi}}{2 \pi \lambda} \sqrt{4 \lambda-\xi^{2}}=4 \int_{-1}^{1} \frac{d x}{2 \pi} e^{2 x \sqrt{\lambda}} \sqrt{1-x^{2}} .
\end{aligned}
$$


Acima, fizemos a mudança de variável $x=\xi /(2 \sqrt{\lambda})$. Este resultado pode ser expresso em termos da função de Bessel $J_{1}(\sqrt{\lambda})$

$$
\left\langle\frac{1}{N} \operatorname{Tr} \exp (M)\right\rangle=\frac{2}{\sqrt{\lambda}} J_{1}(\sqrt{\lambda}), \quad[N \rightarrow \infty],
$$

que é o resultado final para o VEV do laço de Wilson circular no limite de grande $N$. Este resultado é válido para qualquer $\lambda$, ou seja é exato em $\lambda$.

\subsection{Polinômios ortogonais}

Nesta seção, implementaremos outro método para calcularmos o laço de Wilson utilizando modelo de matrizes, o chamado método dos polinômios ortogonais. Este método é, com relação ao anterior, menos geral, por fazer uso específico do potencial em questão. O método consiste basicamente em reescrever o produtório dos autovalores como um produto de polinômios ortogonais, enquanto que o fator de exponencial, que carrega a informação do potencial, é utilizado como medida destes. Apesar de menos geral, este método possui a grande vantagem de nos fornecer um resultado exato, sendo possível calculá-lo em qualquer ordem de $\lambda$ e também de $N$ [26].

Para este método vamos escolher uma outra normalização para $Z$ tal que

$$
Z=\int \prod_{i=1}^{N} d \lambda_{i} \prod_{1 \leq i<j \leq N}\left(\lambda_{j}-\lambda_{i}\right)^{2} e^{-\frac{-2 N}{\lambda} \sum_{i=1}^{N} \lambda_{i}^{2}}
$$

Queremos calcular o VEV do equivalente laço de Wilson

$$
\left\langle\frac{1}{N} \operatorname{Tr} \exp (M)\right\rangle=\frac{1}{Z} \int \prod_{i} d \lambda_{i} \prod_{1 \leq i<j \leq N}\left(\lambda_{j}-\lambda_{i}\right)^{2} \frac{1}{N}\left[\sum_{j} e^{\sqrt{\frac{\lambda}{2 N}} \lambda_{j}}\right] \exp \left[-\sum_{k} \lambda_{k}^{2}\right]
$$

sendo que acima fizemos o reescalonamento dos autovalores, absorvendo os fatores na normalização de $Z$. Primeiramente, vamos observar que podemos escrever o termo de produtório como um determinante dos autovalores, ou seja

$$
\prod_{1 \leq i<j \leq N}\left(\lambda_{j}-\lambda_{i}\right)=\operatorname{det}\left[\left\{\lambda_{i}^{j-1}\right\}\right]
$$


Podemos checar esta identidade de forma mais clara no caso de $N=3$, onde temos que

$$
\left(\lambda_{2}-\lambda_{1}\right)\left(\lambda_{3}-\lambda_{1}\right)\left(\lambda_{3}-\lambda_{2}\right)=\operatorname{det}\left(\begin{array}{ccc}
1 & \lambda_{1} & \lambda_{1}^{2} \\
1 & \lambda_{2} & \lambda_{2}^{2} \\
1 & \lambda_{3} & \lambda_{3}^{2}
\end{array}\right)
$$

A estratégia deste método será escrever este termo do produtório como um produto de polinômios ortogonais dos autovalores $P_{n}\left(\lambda_{i}\right)$. Estes serão ortogonais pela métrica dada pelo termo do potencial, em termos matemáticos queremos

$$
\int_{-\infty}^{\infty} d \lambda_{i} e^{-\lambda_{i}^{2}} P_{n}\left(\lambda_{i}\right) P_{m}\left(\lambda_{i}\right)=\delta_{m n}
$$

Sabemos que tais polinômios, ortonormais por esta métrica, são dados por

$$
P_{n}(\lambda) \equiv \frac{H_{n}(\lambda)}{\sqrt{2^{n} n ! \sqrt{\pi}}}
$$

onde $H_{n}(\lambda)$ são os polinômios de Hermite

$$
H_{n}(\lambda)=e^{-\lambda^{2}}\left(-\frac{d}{d x}\right)^{n} e^{-\lambda^{2}}
$$

Para obtermos estes polinômios a partir de (5.68) nós precisamos apenas fazer processos de reescalonamento de matrizes, que sabemos que por sua vez não alteram o valor do determinante de uma matriz. Também devemos reescalonar os autovalores, que também podemos absorver os fatores na normalização de $Z$. Portanto, podemos escrever o nosso cálculo como

$$
\left\langle\frac{1}{N} \operatorname{Tr} \exp (M)\right\rangle=\frac{1}{Z} \int \prod_{i} d \lambda_{i} \operatorname{det}^{2}\left[\left\{P_{j-1}\left(\lambda_{i}\right)\right\}\right] \frac{1}{N}\left[\sum_{j} e^{\sqrt{\frac{\lambda}{2 N}} \lambda_{j}}\right] \exp \left[-\sum_{k} \lambda_{k}^{2}\right] .
$$

Para as próximas manipulações, vamos analisar o que acontece no caso de $N=3$ e entender como será a generalização do método. Para o caso $N=3$ nós teremos

$$
\frac{1}{Z} \int d \lambda_{1} d \lambda_{2} d \lambda_{3} \operatorname{det}^{2}\left[\left\{P_{j-1}\left(\lambda_{i}\right)\right\}\right] \frac{1}{3}\left(e^{\sqrt{\frac{\lambda}{2 N}} \lambda_{1}}+e^{\sqrt{\frac{\lambda}{2 N}} \lambda_{2}}+e^{\sqrt{\frac{\lambda}{2 N}} \lambda_{3}}\right) e^{-\left(\lambda_{1}^{2}+\lambda_{2}^{2}+\lambda_{3}^{2}\right)}
$$

Vemos que a integral é completamente simétrica com relação aos autovalores $\lambda_{i}$, $\operatorname{logo}$ podemos fazer apenas uma das integrais, geradas pelo termo da soma das exponenciais, e 
multiplicar o nosso resultado por $N=3$, que irá cancelar com o denominador. Portanto temos que resolver

$$
\frac{1}{Z} \int d \lambda_{1} d \lambda_{2} d \lambda_{3} \operatorname{det}^{2}\left[\left\{P_{j-1}\left(\lambda_{i}\right)\right\}\right]\left(e^{-\lambda_{1}^{2}+\sqrt{\frac{\lambda}{2 N}} \lambda_{1}}\right) e^{-\left(\lambda_{2}^{2}+\lambda_{3}^{2}\right)}
$$

Para isto, primeiramente vamos olhar para o fator do determinante dos polinômios

$$
\begin{aligned}
\operatorname{det}^{2}\left[\left\{P_{j-1}\left(\lambda_{i}\right)\right\}\right]= & {\left[P_{0}\left(\lambda_{1}\right) P_{1}\left(\lambda_{2}\right) P_{2}\left(\lambda_{3}\right)+P_{0}\left(\lambda_{2}\right) P_{1}\left(\lambda_{3}\right) P_{2}\left(\lambda_{1}\right)\right.} \\
& +P_{0}\left(\lambda_{3}\right) P_{1}\left(\lambda_{1}\right) P_{2}\left(\lambda_{2}\right)-P_{0}\left(\lambda_{3}\right) P_{1}\left(\lambda_{2}\right) P_{2}\left(\lambda_{1}\right) \\
& \left.-P_{0}\left(\lambda_{2}\right) P_{1}\left(\lambda_{1}\right) P_{2}\left(\lambda_{2}\right)-P_{0}\left(\lambda_{1}\right) P_{1}\left(\lambda_{3}\right) P_{2}\left(\lambda_{2}\right)\right]^{2}
\end{aligned}
$$

Lembrando que vamos fazer a integração dos autovalores $\lambda_{2}$ e $\lambda_{3}$, vemos que somente os termos quadráticos vão contribuir de forma não nula na integração, depois de utilizarmos as relações de ortogonalidade. Logo, após fazer a integração em $\lambda_{2}$ e $\lambda_{3}$, nós temos

$$
\frac{1}{Z} \int d \lambda_{1}\left(\sum_{i=0}^{2} 2 ! P_{i}^{2}\left(\lambda_{1}\right)\right)\left(e^{-\lambda_{1}^{2}+\sqrt{\frac{\lambda}{2 N}} \lambda_{1}}\right)
$$

sendo que o fator de 2 ! (que no caso geral seria $(N-1)$ !) vai cancelar o mesmo fator que aparecerá ao calcular $Z=N$ !.

Para o caso de $N$ qualquer, nós teremos o mesmo procedimento, sendo que para o determinante dos polinômios, nós podemos utilizar a fórmula

$$
\operatorname{det}\left[\left\{P_{j-1}\left(\lambda_{i}\right)\right\}\right]=\sum_{\sigma \epsilon S_{N}} \operatorname{sgn}(\sigma) \prod_{i=1}^{N} P_{\sigma(i)}\left(\lambda_{i}\right)
$$

onde a soma é feita sobre o grupo de permutação $S_{N}$, e $\operatorname{sgn}(\sigma)$ é o sinal da permutação. Para fazer a integração em $\lambda_{1}$ nós vamos utilizar a relação entre estes polinômios e os polinômios de Laguerre $L_{i}$ (confere $7.347 / 7$ em [29])

$$
\int_{-\infty}^{+\infty} d \lambda_{1} P_{i}^{2}\left(\lambda_{1}\right) \exp \left[-\left(\lambda_{1}-\sqrt{\frac{\lambda}{8 N}}\right)^{2}\right]=L_{i}\left(-\frac{\lambda}{4 N}\right)
$$

sendo que para obtermos a exponencial acima, temos apenas que completar quadrados, o que nos dá um fator de $\exp [\lambda / 8 N]$. Finalmente, temos que o resultado através deste método nos dá

$$
\left\langle\frac{1}{N} \operatorname{Tr} \exp (M)\right\rangle=\frac{1}{N} \sum_{i=0}^{N-1} L_{i}(\lambda / 4 N) \exp (\lambda / 8 N)=\frac{1}{N} L_{N-1}^{1}(\lambda / 4 N) \exp (\lambda / 8 N),
$$


sendo que na segunda igualdade utilizamos a relação entre os polinômios de Laguerre generalizados $L_{n}^{(\alpha+1)}(x)=\sum_{i=0}^{n} L_{i}^{\alpha}(x)$. Este resultado é válido para qualquer valor de $\lambda \mathrm{e}$ também de $N$.

Fazendo a expansão de $1 / N$ para $N$ grande, nós temos o resultado

$$
\left\langle\frac{1}{N} \operatorname{Tr} \exp (M)\right\rangle=\frac{2}{\sqrt{\lambda}} J_{1}(\sqrt{\lambda})+\frac{\lambda}{48 N^{2}} J_{2}(\sqrt{\lambda})+\mathcal{O}\left(1 / N^{4}\right),
$$

sendo $J_{1}$ a função de Bessel, onde podemos ver que o primeiro termo coincide exatamente com o resultado (5.64) calculado na seção anterior utilizando o método do ponto de sela (resultado que era válido somente para $N$ grande).

Como fora prometido, eis que chegamos ao resultado exato, expresso em termos de polinômios de Laguerre, para o valor esperado do laço de Wilson circular supersimétrico 1/2 BPS. Mais do que um simples resultado, devemos lembrar que este é um resultado analítico exato e altamente não trivial em uma teoria quântica de campos interagentes. Por tais méritos, este operador é, por si só, um objeto de grande interesse. 


\section{Capítulo 6}

\section{Laços de Wilson holográficos}

Concluído o cálculo do operador na teoria de calibre, agora vamos trabalhar o mesmo operador do ponto do vista do outro lado da dualidade. De fato, a descrição dual deste operador é um dos tópicos que compõe o dicionário da correspondência.

\subsection{Superfícies minimais}

Uma das primeiras dificuldades que encontramos para fazer a descrição dual deste operador é o fato de que na $\mathcal{N}=4$ super Yang-Mills não temos partículas na representação fundamental, temos apenas campos na representação adjunta. Para introduzirmos "quarks", partículas na representação fundamental, vamos imaginar um conjunto sobreposto de $N+1$ D3-branas, sendo que escolhemos uma destas para separar do conjunto, separando-a por uma grande distância. Teremos longas cordas ligadas a esta brana e ao conjunto, que poderão ser interpretadas como "W-bosons", partículas pesadas, ver Figura 6.1. Consequentemente, as pontas destas cordas poderão ser identificadas como quarks pesados, não propagantes.

Do ponto de vista da teoria de campos, este processo será equivalente à uma quebra do grupo $U(N+1) \rightarrow U(1) \times U(N)$, devido a uma partícula escalar adquirindo um grande VEV. Uma vez quebrado o grupo, teremos que as partículas fora da diagonal principal irão se transformar na representação fundamental de $S U(N)$. Em uma representação matricial 


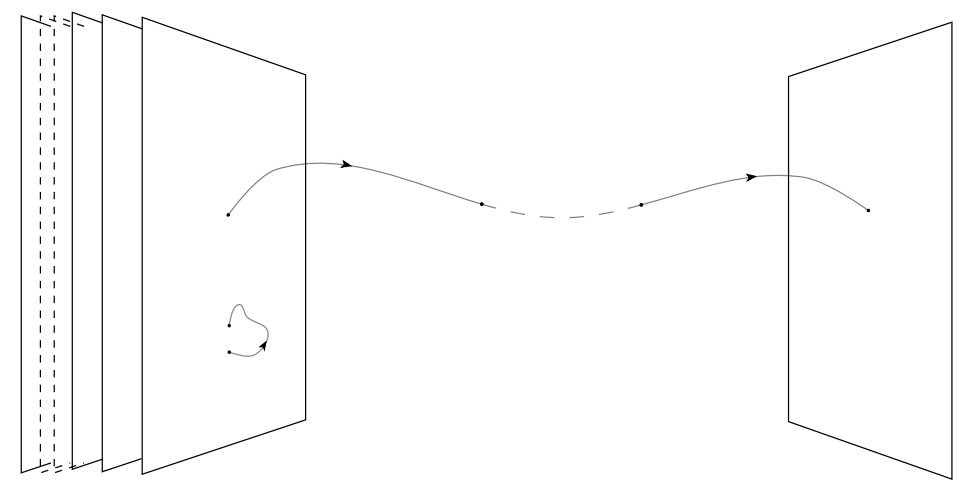

Figura 6.1: Podemos construir objetos na representação fundamental distanciando uma D3-brana de um conjunto de $N$ D3-branas, como ilustrado acima.

podemos decompor o campo de calibre $S U(N+1), \tilde{A}_{M}=\left(\tilde{A}_{\mu}, \tilde{\Phi}_{i}\right)$ na forma

$$
\tilde{A}_{\mu}=\left(\begin{array}{cc}
A_{\mu} & W_{\mu} \\
W_{\mu}^{\dagger} & a_{\mu}
\end{array}\right) \quad \tilde{\Phi}_{i}=\left(\begin{array}{cc}
\Phi_{i} & W_{i} \\
W_{i} & M \phi_{i}
\end{array}\right)
$$

onde $A_{\mu}$ e $\Phi_{i}$ são matrizes $N \times N, W_{\mu}$ e $W_{\mu}^{\dagger}$ são vetores com $N$ componentes e $a_{\mu}$ e $\phi_{i}$ são números. Assim é possível gerar nossos "quarks" muito massivos e não propagantes.

Posto isto, seguimos como foi proposto em [13,30], onde o cálculo dual do laço de Wilson correspondente é dado por

$$
\left\langle W_{C}\right\rangle \sim e^{-S(\partial X=C)},
$$

sendo que para o limite de $\lambda$ grande, esperamos que $S$ seja a área de uma folha mundo de uma corda fundamental presa ao contorno $C$ localizado na borda de $A d S_{5}$ e em um ponto $\theta^{i}$ da $S^{5}$. Pela correspondência, sabemos que tomar o limite $N \rightarrow \infty$, fixando $\lambda$, é equivalente a colocar o acoplamento da corda indo a zero, ou seja, podemos ignorar efeitos de loops das cordas. Agora, tomando também $\lambda \rightarrow \infty$ teremos a tensão da corda indo a infinito, o que nos permite ignorar efeitos de flutuação da superfície da folha mundo. Nestes limites, sabemos que esta folha mundo será uma superfície minimal, ou seja, é solução da ação de Nambu-Goto clássica 


$$
S(C)=S_{N G}=-\frac{1}{2 \pi \alpha^{\prime}} \int_{\partial X=C} d \tau d \sigma \sqrt{\operatorname{det} \gamma_{\alpha \beta}},
$$

sendo que $\gamma_{\alpha \beta}$ é a métrica induzida,

$$
\gamma_{\alpha \beta} \equiv G_{M N} \partial_{\alpha} X^{M} \partial_{\beta} X^{N}
$$

e $G_{M N}$ a métrica do espaço tempo, $A d S_{5} \times S^{5}$ (3.25), que vamos reescrever novamente aqui na forma equivalente em termos das coordenadas imersas $\left(X^{\mu}, Y^{i}\right)$

$$
d s_{A d S_{5} \times S^{5}}^{2}=G_{M N} d X^{M} d X^{N}=\alpha^{\prime} \frac{L^{2}}{Y^{2}}\left(\sum_{\mu=0}^{3} d X^{\mu} d X^{\nu}+\sum_{i=0}^{6} d Y^{i} d Y^{i}\right)
$$

$L$ é como sempre o raio de $A d S_{5}$ e $S^{5}$.

\subsection{Regularização}

Um problema que vemos imediatamente é o fato de que a área desta superfície será divergente, uma vez que a borda desta está localizada em $z=0$. Podemos interpretar esta divergência em termos físicos, lembrando que estamos calculando o laço de Wilson devido a uma partícula na representação fundamental, cuja massa é tal que este possa ser considerado uma fonte não propagante. Podemos interpretar esta divergência como sendo a contribuição da massa da partícula prova. Mas, apesar disso, uma vez que o VEV do laço de Wilson é dado por (6.2) a menos de uma normalização, é natural que possamos eliminar esta divergência. Para fazer a regularização, nós iremos seguir a proposta de [14], onde ao invés de utilizarmos a ação (6.3), nós vamos utilizar uma transformada de Legende desta, ou seja

$$
\tilde{S}=S+\oint d \tau P_{i} Y^{i}(\tau, \sigma=0)
$$

onde

$$
P_{i}=\dot{y}^{i} \frac{\sqrt{\lambda}}{2 \pi Y^{2}}, \quad Y(\sigma=0)=\epsilon,
$$

sendo que $\epsilon$ será o corte da regularização, $y^{i}$ são as coordenadas que se acoplam com os campos escalares e $Y^{i}$ são coordenadas da corda na esfera $S^{5}$. De forma mais clara, nós temos

$$
\tilde{S}=S+\frac{\sqrt{\lambda}}{2 \pi} \oint d \tau \frac{\dot{y}^{i}}{z^{2}} z^{i}=S+\frac{\sqrt{\lambda}}{2 \pi} \oint \frac{|\dot{y}|}{z} d \tau=S+\frac{\sqrt{\lambda}}{2 \pi \epsilon} \oint|\dot{x}| d \tau .
$$


Aqui, utilizamos (6.7) e escolhemos $P^{i}$ paralelo à $z^{i}$, sendo que esta é uma escolha natural para coincidir as condições de contorno para as coordenadas da corda em $S^{5}$ e as coordenadas $y^{i}(\tau)[14]$.

\subsection{Cálculos para linha e círculo e comparação com o modelo de matrizes}

\subsubsection{Cálculo da linha}

Para este cálculo, vamos escolher uma parametrização estática, tal que $\tau=t$ e $\sigma=Y$. Além disso, como dito na seção anterior, as condições de contorno da corda na esfera $S^{5}$ devem coincidir com as coordenadas $y^{i}$. Consequentemente, escolher $y^{1} \neq 0$ e $y^{i \neq 1}=0$ é equivalente a escolher a posição da corda, com relação a $S^{5}$, no polo da esfera. Portanto, podemos omitir as coordenadas da corda sobre a esfera, uma vez que estas serão constantes e irrelevantes para a dinâmica do sistema.

Fazendo esta escolha de parametrização, nós temos que a métrica induzida será

$$
\gamma_{\alpha \beta}=\left(\begin{array}{cc}
-\frac{L^{2}}{\sigma^{2}} & 0 \\
0 & \frac{L^{2}}{\sigma^{2}}
\end{array}\right) .
$$

Podemos expressar a métrica da folha mundo como sendo

$$
d s_{f m}^{2}=\gamma_{\alpha \beta} d \sigma^{\alpha} d \sigma^{\beta}=\frac{L^{2}}{\sigma^{2}}\left(-d \tau^{2}+d \sigma^{2}\right),
$$

Comparando com (3.25), vemos que esta é nada menos do que a métrica $A d S_{2}$.

Substituindo a métrica induzida na ação de Nambu-Goto

$$
S=-\frac{L^{2}}{2 \pi \alpha^{\prime}} \int_{0}^{T} d \tau \int_{\epsilon}^{\infty} \frac{1}{\sigma^{2}} d \sigma=-\frac{\sqrt{\lambda}}{2 \pi \epsilon} T,
$$

onde fizemos uso da relação $L^{2} / \alpha^{\prime}=\sqrt{\lambda}$. Aqui, podemos interpretar essa divergência fisicamente, lembrando que $\langle W\rangle=\exp (-E T)$, sendo que neste caso $E=M$ (massa do quark) é infinita. Portanto, podemos identificar

$$
M=\frac{\sqrt{\lambda}}{2 \pi \epsilon} .
$$


Por outro lado, fazendo o cálculo da regularização, dada por (6.8), utilizando a parametrização do contorno da linha reta (4.17)

$$
\frac{\sqrt{\lambda}}{2 \pi \epsilon} \oint|\dot{x}| d \tau=\frac{\sqrt{\lambda}}{2 \pi \epsilon} \oint d \tau=\frac{\sqrt{\lambda}}{2 \pi \epsilon} T .
$$

Portanto, o resultado para o caso da linha reta, através do cálculo dual nos dá

$$
\left\langle W_{\text {reta }}\right\rangle=\exp \left(S-\frac{\sqrt{\lambda}}{2 \pi \epsilon} T\right)=1,
$$

que coincide exatamente com o resultado da teoria de campos (4.19).

\subsubsection{Cálculo do laço circular}

Queremos agora calcular a área da superfície minimal, cuja fronteira coincida com o círculo de raio $a$, ou seja, para $z=0$ esperamos obter:

$$
X^{i}=(0, a \cos \psi, a \sin \psi, 0), \quad 0 \leq \psi \leq 2 \pi .
$$

Sabendo que através de transformações conformes é possível transformar retas em círculos, nós podemos encontrar esta superfície utilizando a simetria, fazendo uma transformação da forma

$$
\begin{aligned}
X^{\prime i} & =\frac{X^{i}+c^{i}\left(X^{2}+Y^{2}\right)}{1+2 c \cdot X+c^{2}\left(X^{2}+Y^{2}\right)} \\
Y^{\prime} & =\frac{Y}{1+2 c \cdot X+c^{2}\left(X^{2}+Y^{2}\right)},
\end{aligned}
$$

onde aqui $c^{\mu}$ é o parâmetro da transformação conforme para obter a superfície a partir da solução do laço de Wilson de uma linha reta. Outra forma de se obter a superfície minimal para este caso é resolvendo diretamente as equações de movimento, com a condição de contorno circular para a borda da superfície em $Y=0$. Para isto, nós podemos tentar uma solução da forma

$$
\begin{aligned}
X^{i}(\sigma, \tau) & =(0, h(\sigma) \cos \psi, h(\sigma) \sin \psi, 0) \\
Y(\sigma) & =\sigma,
\end{aligned}
$$


juntamente com a condição $h(Y=\sigma=0)=a$, para obtermos a condição de contorno. Com tal parametrização nós temos que

$$
\begin{aligned}
\gamma_{\psi \psi} & =\frac{L^{2}}{\sigma^{2}} h(\sigma)^{2}, \\
\gamma_{\psi \sigma} & =0, \\
\gamma_{\sigma \sigma} & =\frac{L^{2}}{\sigma^{2}}\left(h^{\prime}(\sigma)^{2}+1\right) .
\end{aligned}
$$

Com esta parametrização, temos a seguinte ação de Nambu-Goto

$$
S=-\frac{L^{2}}{2 \pi \alpha^{\prime}} \int d \tau d \sigma \sqrt{\frac{h^{2}}{\sigma^{4}}\left(h^{\prime}(\sigma)^{2}+1\right)},
$$

cuja equação de movimento é

$$
h(\sigma)^{3}\left[-\sigma\left(1+h^{\prime 2}\right)+h\left(-2 h^{\prime}-2 h^{\prime 3}+\sigma h^{\prime \prime}\right)\right]=0 .
$$

Resolvendo esta equação, nós obtemos que

$$
h(Y)=\sqrt{a^{2}-Y^{2}} .
$$

Portanto, temos que a superfície minimal deste contorno é dada por

$$
X^{\prime i}=\sqrt{a^{2}-Y^{2}}(0, \cos \psi, \sin \psi, 0), \quad 0 \leq Y \leq a, 0 \leq \psi \leq 2 \pi,
$$

ver Figura 6.2. Substituindo esta solução na ação, nós obtemos o seguinte resultado

$$
S=-\sqrt{\lambda} \int_{\epsilon}^{a} d \sigma \frac{a}{\sigma^{2}}=\sqrt{\lambda}\left(\frac{a}{\epsilon}-1\right) .
$$

Lembrando que ainda precisamos adicionar o termo de regularização

$$
\frac{\sqrt{\lambda}}{2 \pi \epsilon} \oint|\dot{x}| d \tau=\frac{\sqrt{\lambda}}{\epsilon} a .
$$

Com isto, nós concluímos que o laço de Wilson circular holográfico resulta ser

$$
\left\langle W_{\text {circulo }}\right\rangle_{A d S}=e^{\sqrt{\lambda}} .
$$

Lembrando que o resultado obtido através da teoria de campos foi

$$
\left\langle W_{\text {circulo }}\right\rangle_{\mathrm{CFT}}=\frac{2}{\sqrt{\lambda}} J_{1}(\sqrt{\lambda}),
$$




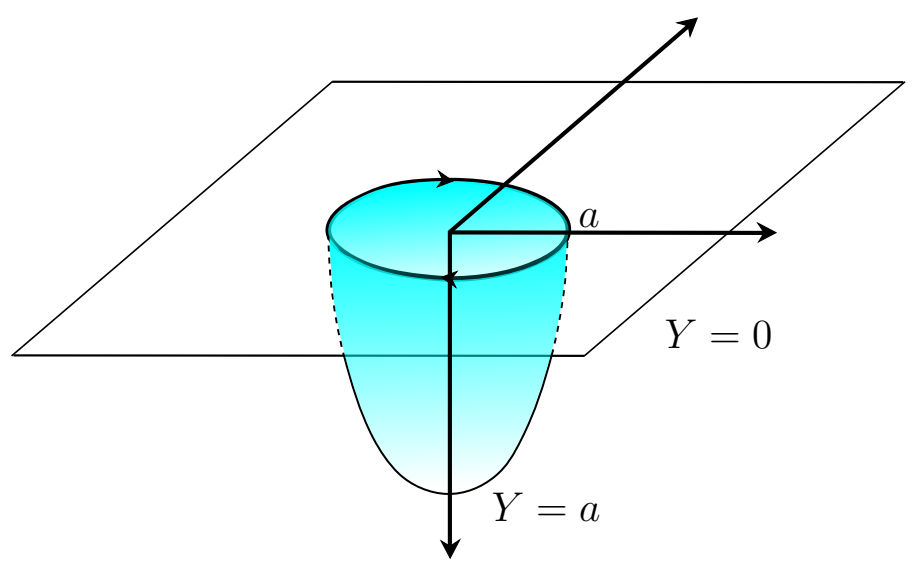

Figura 6.2: Superfície minimal correspondente ao contorno circular.

sendo que este resultado é válido para qualquer $\lambda$, nós podemos tomar o limite de $\lambda \rightarrow \infty$ para compararmos com o resultado holográfico:

$$
\lim _{\lambda \rightarrow \infty}\left\langle W_{\text {circulo }}\right\rangle_{\mathrm{CFT}} \simeq \sqrt{\frac{2}{\pi}} \frac{e^{\sqrt{\lambda}}}{\lambda^{3 / 4}} .
$$

Vemos assim que ambos os resultados nos dão

$$
\left\langle W_{\text {circulo }}\right\rangle \sim e^{\sqrt{\lambda}},
$$

sendo que no caso do cálculo da teoria de campos temos um fator multiplicativo em $\lambda$ que não é reproduzível no nivel semi-clássico da gravidade analizado aqui. Este fator multiplicativo pode ser obtido, no cálculo na AdS, através de flutuações da solução da superfície minimal.

\subsection{Linhas antiparalelas}

Os cálculos apresentados até agora nesta seção puderam ser comparados aos resultados obtidos através da teoria de calibre, onde calculamos operadores com a restrição de serem 1/2 BPS supersimétricos, mas além destes, podemos calcular outros laços de Wilson que não sejam desta classe de operadores. Podemos obter outros operadores fazendo apenas uma mudança no contorno do laço. 
Dentre estes possíveis contornos, vamos observar o que resulta de um contorno retangular, enquanto fixamos a posição na $S^{5}$. De forma muito semelhante ao que fizemos em 2.3 , vamos escolher um retângulo cujo comprimento na direção temporal seja $T$ e a direção espacial perpendicular seja de comprimento $D$. Novamente, vamos supor que $T \gg D$, pois assim poderemos usar o fato de que, para este contorno, o VEV do laço de Wilson nos dá o potencial do campo de calibre na interação de um par de quark-antiquark. De outra forma, teremos que

$$
\langle W(C)\rangle=e^{-E T}=e^{-S_{N G}},
$$

onde $E$ será a energia total deste sistema, que pode ser escrita como $E=2 M+V(D)$, sendo que $V(D)$ é o potencial de interação entre o par e $M$ a massa do quark e antiquark.

Para realizarmos este contorno, vamos escolher convenientemente, de forma coerente com as simetrias do problema, nos ajudando a simplificá-lo. Desta forma, vamos escolher a parametrização das coordenadas da folha-mundo da corda como sendo

$$
\tau=t, \quad \sigma=X_{1},
$$

onde $t$ e $X_{1}$ são coordenadas temporais e espaciais, respectivamente, nas direções do contorno retangular descrito acima. Deixaremos a coordenada $Y$ livre para ser uma função de sigma. As coordenadas das outras duas direções serão escolhidas como sendo constantes, inclusive as referentes à $S^{5}$. Utilizando tais coordenadas como constantes, nós poderemos desprezá-las em nossos cálculos, uma vez que estas não irão acrescentar dinâmica ao sistema.

Esta parametrização vai induzir uma métrica na folha mundo da forma

$$
d s_{f m}^{2}=\frac{L^{2}}{Y^{2}}\left(-d \tau^{2}+\left(1+Y^{\prime 2}\right) d \sigma^{2}\right) .
$$

Tendo escolhido esta parametrização, a única função que devemos achar é $Y=Y(\sigma)$. Para encontrar as equações de movimento para esta função, vamos escrever a ação de Nambu-Goto correspondente

$$
S_{N G}=-\frac{L^{2} T}{2 \pi \alpha^{\prime}} \int_{-D / 2}^{D / 2} d \sigma \frac{1}{Y^{2}} \sqrt{1+Y^{\prime 2}},
$$

com $Y^{\prime}=d Y / d \sigma$. Além disso, nós devemos utilizar a condição de contorno 


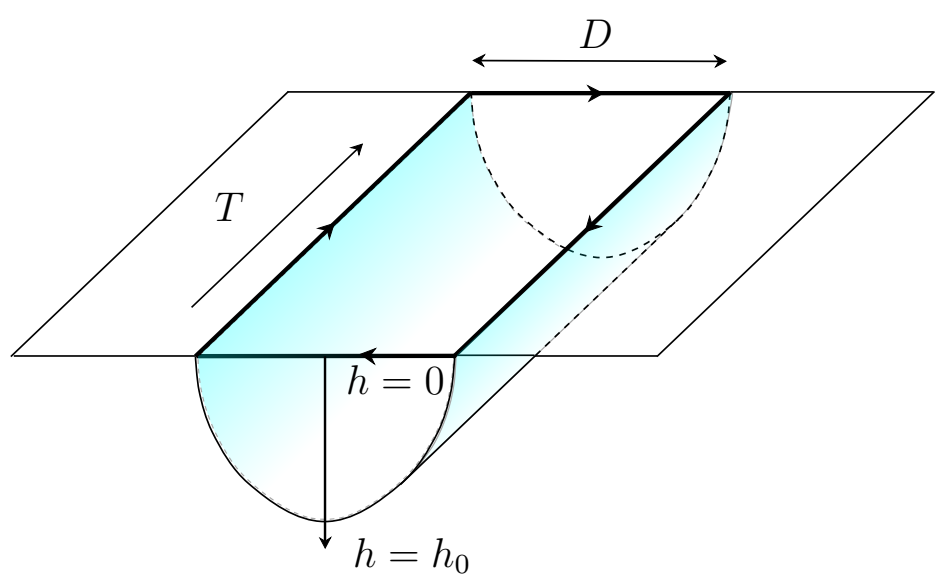

Figura 6.3: Superfície minimal correspondente ao contorno retangular.

$$
Y(D / 2)=Y(-D / 2)=0
$$

que pode ser melhor interpretada olhando para a Figura 6.3. Observando que as condições de contorno são simétricas com relação à mudança de variável $\sigma \rightarrow-\sigma$ e o fato de que a ação em si é simétrica com relação a essa transformação, nós podemos impor que a função $Y(\sigma)$ também seja par. Além disso, vamos fazer uma mudança de variável para obtermos coordenadas adimensionais, definindo

$$
\sigma=D \zeta, \quad \frac{Y(\sigma)}{D}=h(\zeta)
$$

Portanto, considerando a paridade de $Y(\sigma)$ e utilizando coordenadas adimensionais $h(\zeta)$, nós temos que a ação (6.35) pode ser escrita como

$$
S_{N G}=-\frac{2 L^{2}}{2 \pi \alpha^{\prime}} \frac{T}{D} \Lambda
$$

onde definimos

$$
\Lambda \equiv \int_{0}^{1 / 2} \frac{d \zeta}{h^{2}} \sqrt{1+h^{\prime 2}}
$$

que é um fator puramente numérico. Como podemos esperar, este fator numérico é divergente, uma vez que o integrando possui uma divergência em $\zeta=1 / 2$, ou seja, na borda de AdS, como era esperado. Esta divergência será interpretada de maneira mais cuidadosa mais adiante. Feitas tais considerações, podemos derivar a equação de movimento para $h(\zeta)$, encontrar

$$
h^{\prime}=\frac{h_{0}^{4}-h^{4}}{h^{4}}
$$


onde $h_{0}$ é o ponto correspondente ao extremo $h^{\prime}=0$, que, por simetria, se encontra em $\zeta=0$. Podemos encontrar explicitamente o valor de $h_{0}$ através da relação

$$
\frac{1}{2}=\int_{0}^{1 / 2} d \zeta=\int_{0}^{h_{0}} \frac{d h}{h^{\prime}}=\int_{0}^{h_{0}} d \zeta \frac{h^{2}}{\sqrt{h_{0}^{4}-h^{4}}}
$$

sendo que na última passagem fizemos uso da equação de movimento (6.40). Resolvendo esta equação nós temos que

$$
h_{0}=\frac{\Gamma\left(\frac{1}{4}\right)}{2 \sqrt{\pi} \Gamma\left(\frac{3}{4}\right)} .
$$

Agora, podemos escrever $\Lambda$ em termos de $h$ ao invés de $\zeta$, utilizando novamente a equação de movimento (6.40) e (6.41), obtendo

$$
\Lambda=h_{0}^{2} \int_{0}^{h_{0}} \frac{d h}{h^{2} \sqrt{h_{0}^{4}-h^{4}}} .
$$

Como já havíamos comentado, o integrando diverge para $h=0$, sendo necessário portanto estabelecer um corte neste ponto. Como havíamos interpretado em outros casos, vamos estabelecer um corte $Y=0 \rightarrow Y=\epsilon$ (tomando o limite de $\epsilon \rightarrow 0$ no final do cálculo), ou seja um corte na borda da $A d S$. Este corte se traduz em $h=0 \rightarrow h=\epsilon / D$ para as coordenadas adimensionais. Utilizando este corte, podemos calcular o valor de $\Lambda$, resultando em

$$
\Lambda=-\frac{1}{2 h_{0}^{2}}+\frac{D}{\epsilon} .
$$

Podemos usar este resultado e substituir na ação (6.38) o valor $\Lambda$

$$
S_{N G}=\frac{\sqrt{\lambda} T}{\pi D} \frac{1}{2 h_{0}^{2}}-\frac{\sqrt{\lambda}}{\pi} \frac{D}{\epsilon},
$$

onde aqui fizemos uso de que $L^{2} / \alpha^{\prime}=\sqrt{\lambda}$. Para chegar em um resultado livre desta divergência, vamos lembrar que o observável que queremos é $V(D)=E-2 M=-S / T-$ $2 M$, conforme (6.32), onde $M$ é dado por (6.12). Logo, através do cálculo do laço de Wilson no caso de um contorno retangular (linhas antiparalelas), nós podemos concluir que

$$
V(D)=-\frac{\Gamma^{2}\left(\frac{3}{4}\right)}{\Gamma^{2}\left(\frac{1}{4}\right)} \frac{\sqrt{\lambda}}{D}, \quad[\lambda \gg 1],
$$

que é válido para valores grande de $\lambda$. Para valores pequenos de $\lambda$, este mesmo potencial é calculado na teoria de calibre [31], resultando em

$$
V(D)=-\frac{\pi \lambda}{D}, \quad[\lambda \ll 1]
$$


para a ordem mais baixa de $\lambda$. Notemos que o resultado nos diz que a teoria é não confinante, como deve ser em uma teoria conforme, sendo o potencial coulombiano e não linear em $D$. Portanto, temos o potencial entre um par de um quark e um antiquark na $\mathcal{N}=4$ super Yang-Mills com o grupo de calibre $S U(N)$ em quatro dimensões para os limites de $\lambda$ grande e pequenos dados por (6.46) e (6.47), respectivamente, sendo que para valores intermediários de $\lambda$ nós esperamos que o potencial $V(D)$ seja uma função suave que interpole os dois resultados. 


\section{Conclusões}

Neste trabalho, fizemos uma revisão de uma das ferramentes da física teórica atual mais em voga dos últimos tempos, a correspondência AdS/CFT. Além disso, trabalhamos no cálculo de uma importante classe de operadores, os laços de Wilson, no contexto da dualidade, mostrando de forma explícita o poder de utilidade desta. Tais operadores são, de forma geral, de extrema importância em teorias de calibre. Estes nos dão parâmetros de ordem de confinamento/desconfinamento das teorias. Apesar de não ter sido abordado neste trabalho, além disso estes também relacionam-se com invariantes topológicos (por exemplo em teorias de Chern-Simons em 3 dimensões [32]). No contexto da dualidade estudada, estes operadores são calculados em ambos os lados desta, nos proporcionando uma checagem altamente não trivial e importante da correspondência.

Introduzimos o conceito mais geral dos operadores laços de Wilson em teorias de calibre, construindo tais operadores e abordando suas principais características, como a invariância de calibre. Também reproduzimos cálculos destes operadores em teorias simples, no de caso abeliano e não abeliano de uma teoria puramente de calibre.

Motivações para correspondência foram dadas, não somente para o caso estudado, mas de uma forma geral para a dualidades calibre/gravidade. Após apresentarmos o limite de 't Hooft, tratamos da conjectura de Maldacena, motivada pela dupla interpretação física das D-branas. Encerramos a revisão da conjectura apresentando alguns elementos básicos do dicionário da dualidade, como a fórmula GKPW, que relaciona as funções de partição das teorias.

Uma vez apresentados os aspectos básicos da teoria de calibre em questão, definimos os operadores laços de Wilson supersimétricos 1/2 BPS. Como consequência da imposição da supersimetria, limitações ao contorno do laço foram feitas, restringindo aos contornos de 
uma linha reta e circular. Uma vez completamente definida a generalização supersimétrica dos operadores, uma análise perturbativa do VEV destes pôde ser feita, culminando na contagem dos gráficos planares do tipo 'rainbow'.

A fim de proporcionar uma forma de fazer esta contagem, o modelo de matrizes é introduzido, juntamente com as técnicas de polinômios ortogonais e ponto de sela, padrões em cálculos neste contexto. Através destas técnicas, resultados exatos para o VEV dos operadores foram obtidos, algo de extrema raridade no contexto de teorias quânticas de campos em geral.

Por fim, usamos a dualidade para fazer os cálculos dos mesmos operadores através da teoria de cordas, utilizando superfícies minimais, obtendo a concordância com cálculos feitos na teoria de calibre, e assim fazendo uma verificação altamente não trivial da correspondência. Ao final, também elaboramos o cálculo de um caso não supersimétrico, as linhas anti-paralelas.

A descrição dos operadores das teorias de calibre em termos de cordas é apropriado quando a partícula se move ao longo do laço de Wilson transformando-se na representação fundamental do grupo de calibre. Para representações superiores, torna-se [33] necessário trocar as cordas por D-branas, devido ao efeito de Myers [34]. Estas são superfícies de dimensões maiores que cercam a fronteira apoiando-se no laço de Wilson. Essas configurações portam uma carga elétrica proporcional ao rank da representação do laço de Wilson e são chamados de "laços de Wilson gigantes". A abordagem da D-brana é válida quando a representação do laço de Wilson é simétrica ou anti-simétrica e o rank da representação é de ordem $N$. Para representações maiores, onde o rank é de ordem $N^{2}$, a própria abordagem da D-brana precisa ser substituída por uma nova descrição: as D-branas deformam a geometria do espaço, dissolvem-se em fluxos, e tem-se uma transição para uma nova geometria, conhecida como "bubbling geometry" [35]. Esses assuntos não foram abordados nesta dissertação, mas são muito interessantes.

Outros aspectos de laços de Wilson que não foram abordados nesta dissertação são, entre muitas outras coisas, a computação do operator product expansion (OPE) de laços de Wilson [36] e laços de Wilson em outras teorias, como $\mathcal{N}=6$ super Chern-Simons em três dimensões [37]. 


\section{Apêndice I}

\section{Cálculo da função geradora da função de Green do campo eletromagnético}

Aqui, calcularemos explicitamente a função geradora da função de Green do campo eletromagnético, utilizada na equação (2.15). Considerando a ação livre como sendo:

$$
S=\int d^{4} x \mathcal{L}+\int d^{4} x J_{\mu} A^{\mu}=\int d^{4} x\left(\frac{1}{4} F^{\mu \nu} F_{\mu \nu}+J_{\mu} A^{\mu}\right),
$$

onde temos que $F_{\mu \nu}=\partial_{\mu} A_{\mu}-\partial_{\nu} A_{\mu}$. Queremos calcular a seguinte função geratriz:

$$
Z[J]=\int \mathcal{D} A e^{i \int d^{4} x\left(\frac{1}{4} F^{\mu \nu} F_{\mu \nu}+J_{\mu} A^{\mu}\right)},
$$

sendo que ao final das contas, queremos identificar o termo da corrente $\int J_{\mu} A^{\mu} d^{4} x$ com $g \oint_{C} d x_{\mu} A^{\mu}$. Para isso, vamos escrever a ação em termos da transformada de Fourier dos campos

$$
A^{\mu}(x)=\int \frac{d^{4} x}{(2 \pi)^{4}} e^{i k \cdot x} \tilde{A}(k), \quad \tilde{A}^{\mu}(k)=\int d^{4} x e^{-i k \cdot x} A(x) .
$$

Da mesma forma teremos transformações para $J(x)$ e $\tilde{J}(k)$. Substituindo esta transformada, nós teremos a variável $k$ e $k^{\prime}$, uma para cada campo que aparece na ação, junto com um fator de $e^{i x \cdot\left(k+k^{\prime}\right)}$, que podemos utilizar na integral em $d^{4} x$, que nos dará um $\delta\left(k+k^{\prime}\right)$. Fazendo a integração em $k^{\prime}$, nós teremos

$$
S=\frac{1}{2} \int \frac{d^{4} k}{(2 \pi)^{4}}\left[-\tilde{A}_{\mu}(k)\left(k^{2} g^{\mu \nu}-k^{\mu} k^{\nu}\right) \tilde{A}_{\nu}(-k)+\tilde{J}^{\mu}(k) \tilde{A}_{\mu}(-k)\right]
$$


Daqui podemos fazer a mudança de variável:

$$
\tilde{A}_{\mu}(k) \rightarrow \tilde{A}_{\mu}(k)-\frac{\tilde{J}^{\nu}(k) P_{\mu \nu}(k)}{k^{2}-i \epsilon},
$$

onde

$$
P^{\mu \nu}(k)=g^{\mu \nu}-k^{\mu} k^{\nu} / k^{2},
$$

e $\epsilon$ é o parâmetro de corte do propagador.

Com essa mudança de variável, chegamos a

$$
S=\frac{1}{2} \int \frac{d^{4} k}{(2 \pi)^{4}}\left[\tilde{J}_{\mu}(k) \frac{P^{\mu \nu}(k)}{k^{2}-i \epsilon} \tilde{J}_{\nu}(-k)-\tilde{A}_{\mu}(k) k^{2} P^{\mu \nu}(k) \tilde{A}_{\nu}(k)\right] .
$$

O segundo termo nada mais é do que a ação livre, logo, ao fazer a integração de trajetória do campo $\tilde{A}_{\mu}$ o que teremos deste termo é simplesmente a amplitude de transição do vácuo para o vácuo, igual a 1. Portanto, podemos escrever a função de partição como sendo

$$
Z[J]=\exp \left[\frac{i}{2} \int d^{4} x d^{4} y J_{\mu}(x) \Delta^{\mu \nu}(x-y) J_{\nu}(y)\right]
$$

onde

$$
\Delta^{\mu \nu}(x-y)=\int \frac{d^{4} k}{(2 \pi)^{4}} e^{i k \cdot(x-y)} \frac{P^{\mu \nu}(k)}{k^{2}-i \epsilon},
$$

que é o propagador do fóton no calibre de Lorentz, sendo que o propagador de Feynman pode ser obtido facilmente aplicando a conservação de corrente. 


\section{Apêndice II}

\section{Propagadores}

Os propagadores de Feynman utilizados no cálculo de $C_{1}$ em (4.13) são listados abaixo.

\section{Propagador do campo de calibre:}

$$
\Delta_{\mu \nu}^{a b}\left(x_{1}-x_{2}\right)=\left\langle A_{\mu}^{a}\left(x_{1}\right) A_{\nu}^{b}\left(x_{2}\right)\right\rangle=\frac{g_{Y M}^{2} \delta^{a b}}{4 \pi^{2}} \frac{\delta_{\mu \nu}}{\left|x_{1}-x_{2}\right|^{2}} .
$$

\section{Propagador do campo escalar:}

$$
D_{i j}^{a b}\left(x_{1}-x_{2}\right)=\left\langle\Phi_{i}^{a}\left(x_{1}\right) \Phi_{j}^{b}\left(x_{2}\right)\right\rangle=\frac{g_{Y M}^{2} \delta^{a b}}{4 \pi^{2}} \frac{\delta_{i j}}{\left|x_{1}-x_{2}\right|^{2}} .
$$

Estes podem ser obtidos de forma simples através da regras de Feynman da Lagrangiana (3.33) e pelas técnicas padrões de teoria quântica de campos, muito semelhante ao que foi feito na seção 6.4, porém tomando a transformada de Fourier para o espaço de posições, ao invés do espaço de momentos que são mais usuais. 


\section{Apêndice III}

\section{Equação de ponto de sela em termos do resolvente}

Queremos mostrar que a equação

$$
2 f \frac{\rho\left(\xi^{\prime}\right) d \xi^{\prime}}{\xi-\xi^{\prime}}=\frac{1}{\lambda} V^{\prime}(\xi)
$$

implica em

$$
\lim _{\epsilon \rightarrow 0^{+}} \omega(z+i \epsilon)+\lim _{\epsilon \rightarrow 0^{+}} \omega(z-i \epsilon)=-\frac{1}{\lambda} V^{\prime}(z) .
$$

Para isto, vamos fazer o cálculo do valor principal de Cauchy explicitamente:

$$
2 \int \frac{\rho\left(\xi^{\prime}\right) d \xi^{\prime}}{\xi-\xi^{\prime}}=\frac{1}{\lambda} V^{\prime}(\xi)=\lim _{\epsilon \rightarrow 0} 2\left[\int_{-\infty}^{z-i \epsilon} \frac{\rho\left(\xi^{\prime}\right) d \xi^{\prime}}{z-\xi^{\prime}}+\int_{z+i \epsilon}^{+\infty} \frac{\rho\left(\xi^{\prime}\right) d \xi^{\prime}}{z-\xi^{\prime}}\right]
$$

Notemos aqui que devemos tomar o cuidado para não passar o limite para dentro da integral, uma vez que esta operação não estará bem definida. Vamos fazer a mudança de variável: $z-i \epsilon \rightarrow \xi$ no primeiro termo, $z+i \epsilon \rightarrow \xi$ no segundo termo, e assim teremos

$$
\lim _{\epsilon \rightarrow 0} 2\left[\int_{-\infty}^{\xi} \frac{\rho(\xi+i \epsilon) d \xi}{z-\xi-i \epsilon}+\int_{\xi}^{+\infty} \frac{\rho(\xi-i \epsilon) d \xi}{z-\xi+i \epsilon}\right]
$$

Fazendo a expansão $\rho(\xi \pm i \epsilon)=\rho(\xi) \pm i \epsilon \rho^{\prime}(\xi)+O\left(\epsilon^{2}\right)$, ignorando termos de segunda ordem e multiplicando e dividindo pelos complexos conjugados de seus respectivos denominadores, nós temos

$$
\lim _{\epsilon \rightarrow 0} 2\left[\int_{-\infty}^{\xi} \frac{(z-\xi)+i \epsilon}{(z-\xi)^{2}+\epsilon^{2}}\left(\rho(\xi)+i \epsilon \rho^{\prime}(\xi)\right) d \xi+\int_{\xi}^{+\infty} \frac{(z-\xi)-i \epsilon}{(z-\xi)^{2}+\epsilon^{2}}\left(\rho(\xi)-i \epsilon \rho^{\prime}(\xi)\right) d \xi\right] .
$$


Ignorando os termos com fator $\epsilon$ multiplicativo, uma vez que estes vão a zero ao tomarmos o limite, nós ficamos com

$$
\lim _{\epsilon \rightarrow 0} \int_{-\infty}^{+\infty} \frac{2 \rho(\xi)(z-\xi)}{(z-\xi+i \epsilon)(z-\xi-i \epsilon)} d \xi
$$

que pode ser escrito na forma

$$
\lim _{\epsilon \rightarrow 0} \int_{-\infty}^{+\infty} d \xi \rho(\xi)\left[\frac{1}{(z+i \epsilon-\xi)}+\frac{1}{(z-i \epsilon-\xi)}\right]=\lim _{\epsilon \rightarrow 0}-[\omega(z+i \epsilon)+\omega(z-i \epsilon)] .
$$

Outro resultado utilizado foi a inversão da relação do resolvente e a distribuição de auto valores, que pode ser obtida fazendo a integral de resíduos da identidade acima, em outras palavras:

$$
\begin{aligned}
\lim _{\epsilon \rightarrow 0}[\omega(z+i \epsilon)-\omega(z-i \epsilon)] & =\lim _{\epsilon \rightarrow 0} \int_{-\infty}^{+\infty} d \xi \rho(\xi)\left[\frac{1}{(z+i \epsilon-\xi)}-\frac{1}{(z-i \epsilon-\xi)}\right] \\
& =\lim _{\epsilon \rightarrow 0} 2 i \epsilon \int_{-\infty}^{+\infty} d \xi \frac{\rho(\xi)}{(z+i \epsilon-\xi)(z-i \epsilon-\xi)}
\end{aligned}
$$

Por resíduos, nós temos que a integral, independente do contorno escolhido, será

$$
\lim _{\epsilon \rightarrow 0} \pm 2 i \epsilon\left\{2 \pi i\left[\frac{\rho(z \pm i \epsilon)}{ \pm 2 i \epsilon}\right]\right\}=2 \pi i \rho(z)
$$

Portanto vale

$$
\rho(z)=\frac{1}{2 \pi i}[\omega(z+i \epsilon)-\omega(z-i \epsilon)]
$$




\section{Apêndice IV}

\section{Grupo conforme}

Faremos uma breve revisão do grupo conforme, o grupo de isometria do espaço AdS. Uma referência relevante é [38].

O grupo conforme pode ser definido como sendo as transformações de coordenadas que deixam a métrica invariante a menos de um fator de escala, ou seja,

$$
g_{\mu \nu}^{\prime}\left(x^{\prime}\right)=\Lambda(x) g_{\mu \nu}(x) .
$$

Como consequência desta definição, vemos que o grupo de Poincaré é especial, caso de transformações que $\Lambda(x)=1$, logo, este é um subgrupo do grupo conforme. Para transformações infinitesimais $x^{\prime \mu}=x^{\mu}+\epsilon^{\mu}(x)$ sabemos que a métrica se transforma na forma

$$
\begin{aligned}
g_{\mu \nu}^{\prime}(x) & =\frac{\partial x^{\alpha}}{\partial x^{\prime \mu}} \frac{\partial x^{\beta}}{\partial x^{\prime \nu}} g_{\alpha \beta} \\
& =\left(\delta_{\mu}^{\alpha}-\partial_{\mu} \epsilon^{\alpha}\right)\left(\delta_{\nu}^{\beta}-\partial_{\nu} \epsilon^{\beta}\right) g_{\alpha \beta} \\
& =g_{\mu \nu}-\left(\partial_{\mu} \epsilon_{\nu}+\partial_{\nu} \epsilon_{\mu}\right)+\mathcal{O}\left(\epsilon^{2}\right) .
\end{aligned}
$$

Ao impormos a relação (1) na equação acima e escrevendo $\Lambda(x)=1+f(x)$, nós temos que

$$
\partial_{\mu} \epsilon_{\nu}+\partial_{\nu} \epsilon_{\mu}=f(x) g_{\mu \nu}
$$

Podemos expressar a função $f(x)$ em termos de $\epsilon$ tomando o traço da equação acima

$$
f(x)=\frac{2}{d} \partial_{\rho} \epsilon^{\rho},
$$

onde estamos denotando por $d$ o número de dimensões do espaço, e portanto $g_{\mu \nu} g^{\mu \nu}=$ $\delta_{\mu}^{\mu}=d$. Para simplificar os cálculos, podemos usar a métrica Minkowski $\eta_{\mu \nu}$ ao invés de 
$g_{\mu \nu}$. Substituindo $f(x)$ em (3) e aplicando a derivada $\partial^{\mu}$, obtemos

$$
\partial^{2} \epsilon_{\nu}+\left(1-\frac{2}{d}\right) \partial_{\nu} \partial_{\rho} \epsilon^{\rho}=0
$$

Podemos aplicar $\partial_{\mu}$ na equação acima e $\partial^{2}$ na equação (3) para obter

$$
(2-d) \partial_{\mu} \partial_{\nu} \partial_{\rho} \epsilon^{\rho}=\partial^{2} \partial_{\rho} \epsilon^{\rho} \eta_{\mu \nu}
$$

e finalmente tomando o traço desta equação nós temos que

$$
(1-d) \partial^{2} \partial_{\rho} \epsilon^{\rho}=0
$$

que nos diz que $\epsilon$ pode ser no máximo quadrático em $x$. Se tentarmos encontrar uma solução da forma

$$
\epsilon_{\mu}=a_{\mu}+b_{\mu \nu} x^{\nu}+c_{\mu \nu \rho} x^{\rho} x^{\nu}
$$

as condições acima implicam que os coeficientes devam ser

- $a_{\mu}$ um vetor constante qualquer, correspondendo às translações.

- $b_{\mu \nu} x^{\nu}=\lambda x_{\mu}+\omega_{\mu \nu} x^{\nu}$, sendo $\lambda$ uma constante qualquer e $\omega_{\mu \nu}=-\omega_{\nu \mu}$, correspondendo respectivamente a dilatações e rotações.

- $c_{\mu \nu \rho} x^{\nu} x^{\rho}=2\left(x_{\rho} b^{\rho}\right) x_{\mu}-b_{\mu} x^{\rho} x_{\rho}$, correspondendo a transformações especiais conformes (TEC).

As três primeiras transformações são bem conhecidas, enquanto que a última, a princípio, tem interpretação física menos óbvia. Uma forma de visualizar melhor esta transformação é escrevendo-a na como

$$
\frac{x^{\prime \mu}}{x^{\prime 2}}=\frac{x^{\mu}}{x^{2}}-b^{\mu}
$$

que podemos entender como sendo uma translação precedida e procedida por uma inversão da forma $x^{\mu} \rightarrow x^{\mu} / x^{2}$. Os seus respectivos geradores podem ser listados como:

$$
\begin{aligned}
\operatorname{translação~} & P_{\mu} & =-i \partial_{\mu} \\
\text { dilatação } & D & =-i x^{\mu} \partial_{\mu} \\
\text { rotação } & M_{\mu \nu} & =i\left(x_{\mu} \partial_{\nu}-x_{\nu} \partial_{\mu}\right) \\
\text { TEC } & K_{\mu} & =-i\left(2 x_{\mu} x^{\nu} \partial_{\nu}-x^{2} \partial_{\mu}\right),
\end{aligned}
$$


cuja respectiva álgebra é

$$
\begin{aligned}
{\left[D, P_{\mu}\right] } & =i P_{\mu}, \\
{\left[D, K_{\mu}\right] } & =-i K_{\mu} \\
{\left[K_{\mu}, P_{\nu}\right] } & =2 i\left(\eta_{\mu \nu} D-M_{\mu \nu}\right), \\
{\left[K_{\rho}, M_{\mu \nu}\right] } & =i\left(\eta_{\rho \mu} K_{\nu}-\eta_{\rho \nu} P_{\mu}\right), \\
{\left[P_{\rho}, M_{\mu \nu}\right] } & =i\left(\eta_{\rho \mu} P_{\nu}-\eta_{\rho \nu} P_{\mu}\right), \\
{\left[M_{\mu \nu}, M_{\rho \sigma}\right] } & =i\left(\eta_{\nu \rho} M_{\mu \sigma}+\eta_{\mu \sigma} M_{\nu \rho}-\eta_{\mu \rho} M_{\nu \sigma}-\eta_{\nu \sigma} M_{\mu \rho}\right) .
\end{aligned}
$$

Como dito na sessão (3.3), o grupo conforme em 4 dimensõs é isomórfico ao grupo $S O(2,4)$. Isto pode ser visto definindo

$$
\begin{array}{ll}
J_{\mu \nu}=M_{\mu \nu}, & J_{-1, \mu}=\frac{1}{2}\left(P_{\mu}-K_{\mu}\right), \\
J_{-1,0}=D, & J_{0, \mu}=\frac{1}{2}\left(P_{\mu}+K_{\mu}\right),
\end{array}
$$

com os índices $a, b \in\{-1,0,1, \ldots, 4\}$ e verificando que estes geradores obedecem a álgebra

$$
\left[J_{a b}, J_{c d}\right]=i\left(\eta_{a d} J_{b c}+\eta_{b c} J_{a d}-\eta_{a c} J_{b d}-\eta_{b d} J_{a c}\right)
$$

que define a álgebra dos geradores do grupo $S O(2,4)$. Lembrando que além da simetria bosônica, a $\mathcal{N}=4$ super Yang-Mills em 4 dimensões ainda possui a simetria-R, cujos geradores podemos representar por $T^{A}(A=1, \ldots, 15)$, supersimetria de Poincaré, $\bar{Q}_{\dot{\alpha} a} \mathrm{e}$ $Q_{\alpha}^{a}$, e supersimetrias conformes, $\bar{S}_{\dot{\alpha}}^{a}$ e $S_{\alpha a}$. As supersimetrias conformes aparecem como resultado de $\left[Q, K_{\mu}\right] \neq 0$, e portanto também fazem parte da álgebra supersimétrica. Podemos resumir o conjunto de geradores de simetria de $S U(2,2 \mid 4)$ na forma esquemática

$$
\left(\begin{array}{cc}
P_{\mu}, K_{\mu}, M_{\mu \nu}, D & Q_{\alpha}^{a}, \bar{S}_{\dot{\alpha}}^{a} \\
\bar{Q}_{\dot{\alpha} a}, S_{\alpha a} & T^{A}
\end{array}\right),
$$

com as simetrias bosónicas na diagonal e as fermiónicas fora da diagonal. 


\section{Referências Bibliográficas}

[1] J. M. Maldacena, "The large $N$ limit of superconformal field theories and supergravity," Adv. Theor. Math. Phys. 2, 231 (1998) [Int. J. Theor. Phys. 38, 1113 (1999)] [arXiv:hep-th/9711200].

[2] S. S. Gubser, I. R. Klebanov and A. M. Polyakov, "Gauge theory correlators from non-critical string theory," Phys. Lett. B 428, 105 (1998) [arXiv:hep-th/9802109].

[3] E. Witten, "Anti-de Sitter space and holography," Adv. Theor. Math. Phys. 2, 253 (1998) [arXiv:hep-th/9802150].

[4] O. Aharony, S. S. Gubser, J. M. Maldacena, H. Ooguri and Y. Oz, "Large N field theories, string theory and gravity," Phys. Rept. 323, 183 (2000) [arXiv:hep-th/9905111].

[5] I. R. Klebanov, "TASI lectures: Introduction to the AdS/CFT correspondence," arXiv:hep-th/0009139.

[6] J. M. Maldacena, "Lectures on AdS/CFT," arXiv:hep-th/0309246.

[7] G. T. Horowitz and J. Polchinski, "Gauge / gravity duality," arXiv:gr-qc/0602037.

[8] J. McGreevy, "Holographic duality with a view toward many-body physics," arXiv:0909.0518 [hep-th].

[9] S. W. Hawking, "Particle Creation By Black Holes," Commun. Math. Phys. 43, 199 (1975) [Erratum-ibid. 46, 206 (1976)].

[10] T. P. Cheng and L. F. Li, "Gauge Theory Of Elementary Particle Physics," Oxford, Uk: Clarendon ( 1984) 536 P. ( Oxford Science Publications) 
[11] G. W. Semenoff and K. Zarembo, "Wilson loops in SYM theory: From weak to strong coupling," Nucl. Phys. Proc. Suppl. 108, 106 (2002) [arXiv:hep-th/0202156].

[12] V. Pestun, "Localization of gauge theory on a four-sphere and supersymmetric Wilson loops," Commun. Math. Phys. 313, 71 (2012) [arXiv:0712.2824 [hep-th]].

[13] J. M. Maldacena, "Wilson loops in large N field theories," Phys. Rev. Lett. 80, 4859 (1998) [arXiv:hep-th/9803002].

[14] N. Drukker, D. J. Gross and H. Ooguri, "Wilson loops and minimal surfaces," Phys. Rev. D 60, 125006 (1999) [hep-th/9904191].

[15] M. Srednicki, "Quantum field theory," Cambridge, UK: Univ. Pr. (2007) 641 p.

[16] S. Weinberg and E. Witten, "Limits on Massless Particles," Phys. Lett. B 96, 59 (1980).

[17] G. 't Hooft, "A Planar Diagram Theory for Strong Interactions," Nucl. Phys. B 72, $461(1974)$.

[18] J. Polchinski, "Dirichlet Branes and Ramond-Ramond charges," Phys. Rev. Lett. 75, 4724 (1995) [hep-th/9510017].

[19] J. Casalderrey-Solana, H. Liu, D. Mateos, K. Rajagopal and U. A. Wiedemann, "Gauge/String Duality, Hot QCD and Heavy Ion Collisions," arXiv:1101.0618 [hepth].

[20] E. D'Hoker and D. Z. Freedman, "Supersymmetric gauge theories and the AdS / CFT correspondence," hep-th/0201253.

[21] M. F. Sohnius and P. C. West, "Conformal Invariance in N=4 Supersymmetric YangMills Theory," Phys. Lett. B 100 (1981) 245; M. T. Grisaru, M. Rocek and W. Siegel, "Zero Three Loop Beta Function In N=4 Super Yang-Mills Theory," Phys. Rev. Lett. 45, 1063 (1980); M. T. Grisaru and W. Siegel, "Supergraphity. 2. Manifestly Covariant Rules and Higher Loop Finiteness," Nucl. Phys. B 201 (1982) 292 [Erratum-ibid. B 206 (1982) 496]; L. Brink, O. Lindgren and B. E. W. Nilsson, "N=4 Yang-Mills 
Theory on the Light Cone," Nucl. Phys. B 212 (1983) 401; L. Brink, O. Lindgren and B. E. W. Nilsson, "The Ultraviolet Finiteness of the N=4 Yang-Mills Theory," Phys. Lett. B 123 (1983) 323.

[22] S. W. Hawking, "The Chronology protection conjecture," Phys. Rev. D 46, 603 (1992).

[23] M. M. Caldarelli, D. Klemm and P. J. Silva, Class. Quant. Grav. 22, 3461 (2005) [hep-th/0411203].

[24] M. Bianchi, M. B. Green and S. Kovacs, "Instanton corrections to circular Wilson loops in N=4 supersymmetric Yang-Mills," JHEP 0204, 040 (2002) [hep-th/0202003].

[25] J. K. Erickson, G. W. Semenoff and K. Zarembo, "Wilson loops in N=4 supersymmetric Yang-Mills theory," Nucl. Phys. B 582, 155 (2000) [hep-th/0003055].

[26] N. Drukker and D. J. Gross, "An Exact prediction of N=4 SUSYM theory for string theory," J. Math. Phys. 42, 2896 (2001) [hep-th/0010274].

[27] P. Di Francesco, P. H. Ginsparg and J. Zinn-Justin, "2-D Gravity and random matrices," Phys. Rept. 254, 1 (1995) [hep-th/9306153].

[28] M. Marino, "Les Houches lectures on matrix models and topological strings," hepth/0410165.

[29] I. Gradshteyn and I. Ryzhik, "Tables of Integrals, Sums, and Products," Academic Press (2000).

[30] S. J. Rey and J. T. Yee, "Macroscopic strings as heavy quarks in large N gauge theory and anti-de Sitter supergravity," Eur. Phys. J. C 22, 379 (2001) [arXiv:hepth/9803001].

[31] J. K. Erickson, G. W. Semenoff, R. J. Szabo and K. Zarembo, "Static potential in N=4 supersymmetric Yang-Mills theory," Phys. Rev. D 61, 105006 (2000) [hepth/9911088].

[32] E. Witten, "Quantum field theory and the Jones polynomial," Commun. Math. Phys. 121, 351 (1989). 
[33] N. Drukker and B. Fiol, "All-genus calculation of Wilson loops using D-branes," JHEP 0502, 010 (2005) [arXiv:hep-th/0501109]; S. Yamaguchi, "Wilson loops of anti-symmetric representation and D5-branes," JHEP 0605, 037 (2006) [arXiv:hepth/0603208]; K. Okuyama and G. W. Semenoff, "Wilson Loops In N $=4$ Sym And Fermion Droplets," JHEP 0606, 057 (2006) [arXiv:hep-th/0604209]; J. Gomis and F. Passerini, "Holographic Wilson loops," JHEP 0608, 074 (2006) [arXiv:hepth/0604007]; "Wilson loops as D3-branes," JHEP 0701, 097 (2007) [arXiv:hepth/0612022].

[34] R. C. Myers, "Dielectric-branes," JHEP 9912, 022 (1999) [arXiv:hep-th/9910053].

[35] O. Lunin, "On gravitational description of Wilson lines," JHEP 0606, 026 (2006) [hep-th/0604133]; E. D'Hoker, J. Estes and M. Gutperle, "Gravity duals of halfBPS Wilson loops," JHEP 0706, 063 (2007) [arXiv:0705.1004 [hep-th]]; T. Okuda and D. Trancanelli, "Spectral curves, emergent geometry, and bubbling solutions for Wilson loops," JHEP 0809, 050 (2008) [arXiv:0806.4191 [hep-th]].

[36] D. E. Berenstein, R. Corrado, W. Fischler and J. M. Maldacena, "The Operator product expansion for Wilson loops and surfaces in the large N limit," Phys. Rev. D 59, 105023 (1999) [hep-th/9809188]; S. Giombi, R. Ricci and D. Trancanelli, "Operator product expansion of higher rank Wilson loops from D-branes and matrix models," JHEP 0610, 045 (2006) [hep-th/0608077]; J. Gomis, S. Matsuura, T. Okuda and D. Trancanelli, "Wilson loop correlators at strong coupling: From matrices to bubbling geometries," JHEP 0808, 068 (2008) [arXiv:0807.3330 [hep-th]].

[37] N. Drukker and D. Trancanelli, "A Supermatrix model for N=6 super Chern-Simonsmatter theory," JHEP 1002, 058 (2010) [arXiv:0912.3006 [hep-th]].

[38] P. Di Francesco, P. Mathieu and D. Senechal, "Conformal field theory," New York, USA: Springer (1997) 890 p 